UNIVERSIDAD AUTÓNOMA METROPOLITANA U-IZTAPALAPA DIVISIÓN DE CIENCIAS BÁSICAS E INGENIERÍA

\title{
Teoría de Norma Gravitacional No-Conmutativa
}

Tesis que presenta:

Ing. Marco Antonio Alcántara Aguilar

Para obtener el grado de:

Maestro en Ciencias (Física)

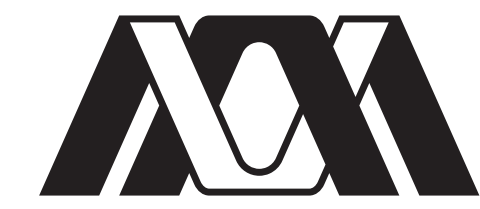

Casa abierta al tiempo

Asesor:

Dr. Marco Antonio Maceda Santamaría

Jurado Calificador:

Presidente: $\quad$ Dra. Nora Eva Bretón Báez

Secretario Dr. Marco Antonio Maceda Santamaría

Vocal: $\quad$ Dr. Hugo Aurelio Morales Técotl

Ciudad de México, Diciembre de 2018 



\section{Índice general}

Índice general

$\begin{array}{lll}\text { Agradecimientos } & \text { III }\end{array}$

Resumen $\quad$ V

1 Introducción 1

2 Teoría de Norma 5

2.1. Teoría de Norma No-Abeliana . . . . . . . . . . . . . . . . . 5

2.2. Teoría de Norma en Electromagnetismo . . . . . . . . . . . . . . . . 7

2.3. Teoría de Norma en Gravitación . . . . . . . . . . . . . . . . . . . . . . . . 10

2.4. Elementos de la Geometría Diferencial . . . . . . . . . . . . . . . . . . 11

2.5. El grupo de Poincaré como simetría interna . . . . . . . . . . . . 23

3 Deformación de la Teoría de Norma para Gravedad 33

3.1. Producto de Moyal-Weyl . . . . . . . . . . . . . . . . . . . . . . 33

3.2. Aplicación de Seiberg-Witten . . . . . . . . . . . . . . . . . . . . 34

3.3. Grupo de simetrías no-conmutativas . . . . . . . . . . . . . . . . . 36

4 Teoría Gravitacional No-Conmutativa $\quad 39$

4.1. BTZ No-Conmutativo . . . . . . . . . . . . . . . . . . . . . . . . 39

4.2. Kerr-Newman No-Conmutativo . . . . . . . . . . . . . . . . . 41

5 Potencial Efectivo No-Conmutativo en BTZ 47

6 Conclusiones y Perspectivas $\quad 57$

$\begin{array}{lr}\text { Bibliografía } & 59\end{array}$ 



\section{Agradecimientos}

Escribo estas breves palabras para agradecer a mis padres Pedro y María del Socorro por su gran apoyo y dedicación por hacer de mí una mejor persona, agradezco a mi esposa Zyania por su consejo y comprensión en los largos días de estudio.

También agradezco al Dr. Marco Antonio Maceda Santamaría que mediante su enseñanza e instrucción hizo posible la conformación de este proyecto como mi asesor de tesis.

De igual manera agradezco a la Universidad Autónoma Metropolitana por brindarme la oportunidad de ser parte de su comunidad así como a amigos y compañeros en especial a Eric, con quien el estudio y la discusión se hacian mas amenos.

Por último agradezco el apoyo recibido por CONACyT, que a través del programa nacional de becas de posgrado hizo posible el estudio de esta maestría. 



\section{Resumen}

El estudio de la Física ha experimentado grandes cambios con la formulación de ideas y conceptos que han ayudado a entender mejor los fenómenos que ocurren a nuestro alrededor; una de estas ideas fue la teoría de norma que puso en escena la importancia de la simetría local [1]. La incorporación del principio de norma con la teoría de gravitación ha llevado a comprender la relación entre la simetría local con el principio de equivalencia, que hace posible la incorporación de elementos que facilitan el entendimiento de la teoría gravitacional.

Otra de las ideas que han producido grandes avances en la física es el álgebra noconmutativa; W. Heisenberg propuso la idea de tener un conmutador diferente de cero en este caso de dos operadores $[\hat{x}, \hat{p}]=i \hbar[2]$ lo que permitió obtener una interpretación diferente que logrará dar sentido a fenómenos microscópicos. Basado en esta idea, Snyder propuso un álgebra no-conmutativa para el espacio-tiempo, que se basan en la siguiente relación de conmutación $\left[x^{\mu}, x^{\nu}\right]=-i \Theta^{\mu \nu}$, con el propósito de resolver problemas de divergencias [3].

Entender nuestro entorno no siempre es una tarea sencilla. Realizar esta ardua labor implica trabajar bajo conceptos, ideas y teorías que llevan a un mejor entendimiento de la naturaleza. En este trabajo de tesis vamos a trabajar con una teoría de norma para gravedad que será sujeta a una deformación del álgebra que la describe. La motivación de calcular una teoría de norma deformada para gravedad tiene como objetivo observar las características de la métrica, los campos de norma y el potencial efectivo de partículas prueba.

Estudiaremos dos espacios tiempos con métricas de hoyos negros con parámetro de rotación en 3 y 4 dimensiones espacio temporales las cuales son: BTZ [4] y Kerr-Newman [5] respectivamente. A continuación se describe los pasos a seguir: se empieza por analizar las transformaciones inducidas por el grupo de Poincaré, lo cual conduce a dos campos de norma, uno relacionado a las traslaciones $e_{\mu}^{a}$ y otro a las rotaciones $\omega_{\mu}^{a b}$. Con estos elementos construimos nuestra teoría de norma para gravedad. Para la deformación vamos a utilizar la aplicación de Seiberg-Witten [6], que nos define los campos de norma deformados, necesarios para la construicción de la métrica deformada.

La deformación de la teoría de gravedad permite considerar la no existencia de puntos en el espacio sino regiones con una escala determinada por el parámetro de deformación. Por eso parte de este trabajo es investigar las consecuencias en la deformación del potencial efectivo de partículas prueba, y a su vez establecer un camino para determinar la teoría de norma no-conmutativa para gravedad. 


\section{Capítulo 1}

\section{Introducción}

El principio de norma ha permitido comprender la importancia de la simetría inherente en los fenómenos de la naturaleza que se ha relacionado con las fuerzas fundamentales del universo. Avances importantes en la teoría electromagnética, electrodinámica cuántica, gravitación, se han producido gracias a este principio.

Una primera visión de la teoría de norma fue abordada por Hermann Weyl a inicios del siglo XX [7], quien inspirado en la teoría de la Relatividad General de Einstein realizó su revolucionaria idea de invarianza de norma. Aunque no fue bien recibida en su época, la esencia de la teoría de norma se mantuvo presente en la teoría electromagnética como una característica de las ecuaciones de Maxwell y que por lo tanto puede identificarse como una teoría de norma; de esta forma se mantiene viva la idea de H. Weyl. Posteriormente con el desarrollo de la mecánica cuántica se visualizó que la teoría de norma podría ser reinterpretada [1]. En este contexto se concibe la invarianza como una que ocurre bajo las transformaciones de fase,

$$
\psi \longrightarrow \psi e^{-i e \lambda}
$$

A mediados del siglo XX la teoría de norma encuentra una nueva vía de explicación gracias a la propuesta de C.N. Yang y R. Mills [8] de buscar una descripción de la interacción nuclear fuerte como una teoría de campo invariante de norma. Esto los llevo a considerar a $S U(2)$ como el grupo de norma local asociado con una rotación en 3-dimensiones $R(\theta) \psi=e^{-i \theta L} \psi$ del espacio del spín isotópico. Para garantizar invarianza de esta cantidad el potencial de norma tiene que ser proporcional al momento angular $L_{i}$, por lo tanto la forma del potencial de norma es $A_{\mu}=\sum_{i} A_{\mu}^{i}(x) L_{i}$. Por lo que en consecuencia ahora el grupo $S U(2)$ es visto como el grupo de simetrías local que se asocia con un campo de norma. Bajo la idea de Yang y Mills se puede construir una teoría de norma para gravedad considerando el grupo global de Poincaré como el grupo de smetría local; esto proporcionará la estructura matemática para obtener una forma geométrica alternativa a la usual en forma métrica de la relatividad general y además, como veremos más adelante, los campos de norma ${ }^{1}$ que resulten de esta teoría facilitarán el cálculo de las ecuaciones de campo.

Para el caso del álgebra no-conmutativa, una de las principales ideas de la no conmutatividad surge a finales de los años veinte y principios de los años treinta con la formulación

\footnotetext{
${ }^{1}$ Campo de norma de traslación $e_{\mu}^{a}$, campo de norma de rotaciones $\omega_{\mu}^{a b}$
} 
de W. Heisenberg [2] de su famoso conmutador de operadores $[\hat{x}, \hat{p}]=i \hbar$, que agregó un nuevo elemento a la mecánica cuántica para explicar la fenomenología alrededor de esta. A mediados de la década de los cuarentas, H. Groenewold y J. Moyal [9] proponen una nueva forma de interpretar la mecánica cuántica usando un producto estrella con el propósito de tener una conexión más natural e intuitiva para el límite clásico, manteniendo la interpretación clásica de las variables. Bajo la idea de no-conmutatividad, H. Snyder [10] propuso la idea de que es posible construir un espacio-tiempo discreto invariante de Lorentz, lo que deriva en relaciones de conmutación entre operadores espacio temporales $x, y, z \mathrm{y} t$, esto con la intención de remover los problemas de divergencia en la teoría de campos. En general se pueden considerar relaciones de conmutación del tipo

$$
\left[x^{\mu}, x^{\nu}\right]=i \Theta^{\mu \nu},
$$

entre las coordenadas espacio-tiempo con una matríz $\Theta^{\mu \nu}$ antisimétrica.

Retomando a la teoría de norma, N. Seiberg y E. Witten [11], a partir de la idea de no-conmutatividad [10], construyen una teoría de campo no-conmutativa. Como una consecuencia de esta se observó que la teoría de norma no-conmutativa aparece como un cierto límite a la teoría de cuerdas; este resultado sugiere algo muy interesante, que la construcción de la teoría de norma no-conmutativa se puede dar a partir de la teoría de norma sin deformar a tráves de una aplicación conocida como de Seiberg-Witten [6].

La incursión en nuevas ideas nos ayuda a ver los problemas desde diferentes perspectivas para poder revelar la mayor cantidad de elementos que nos ayuden a resolver y entender cómo se comporta nuestro entorno. Una de estas ideas es la teoría de norma no-conmutativa para gravedad. En el presente trabajo se estudiará una teoría de norma no-conmutativa para gravedad correspondiente a los espacio-tiempos de BTZ y Kerr-Newmann para observar cómo afecta el término de la deformación tanto a los campos de norma, la métrica y el potencial efectivo asociado al movimiento orbital de partículas prueba. La motivación para escoger estas métricas viene del hecho que contienen términos de rotación que proporcionan una idea más apegada a la realidad del comportamiento de los hoyos negros, y cómo éstos se verán afectados después de la deformación. A continuación describiremos la estructura general del presente trabajo.

En el segundo capítulo plantemos una teoría de norma gravitacional, empezando con una introducción a la teoría de norma en general, para después incorporar elementos de la geometría diferencial, que junto con la idea de transformaciones infinitesimales para el grupo de Poincaré, permitirá obtener los campos de norma $e_{\mu}^{a}$ y $\omega_{\mu}^{a b}$, con los cuales se construye la teoría de norma gravitacional.

En el tercer capítulo se dará una breve introducción al producto Moyal que da lugar a un álgebra no-conmutativa, como ingrediente necesario para la formulación de la teoría de Seiberg-Witten, que ejemplifica una forma adecuada para obtener una teoría de norma no-conmutativa a partir de la teoría de norma estándar, mediante la aplicación de SeibergWitten.

En el capítulo 4 se presenta la aplicación de Seiberg-Witten para BTZ y Kerr-Newman, con la ayuda del programa de cálculo Mathematica, por lo que se determinarán los campos de norma y la métrica deformada hasta segundo orden en el parámetro de deformación $\Theta^{\mu \nu}$ para su análisis posterior. 
Por último, en el capítulo 5, a partir de la métrica deformada, calcularemos la forma del Lagrangiano de partículas prueba del cual vamos a obtener la forma del potencial efectivo deformado asociado al movimiento orbital de partículas prueba. Posteriormente se fijarán los valores de los parámetros en las soluciones deformadas para obtener gráficas a diferentes valores de la masa del hoyo negro, parámetro de rotación y momento angular, tanto para partículas masivas como para no masivas. Con estos resultados compararemos el potencial efectivo deformado de partículas prueba y el no deformado para observar como afecta la deformación a la teoría gravitacional. Finalmente, presentaremos algunas conclusiones y perspectivas que pueden seguirse en trabajo futuro. 



\section{Capítulo 2}

\section{Teoría de Norma}

Uno de los principales conceptos en la teoría de norma se basa en el análisis de la simetría de grupo, ya sea relacionado con la geometría del espacio-tiempo o con la naturaleza de las partículas, que permite reformular con ayuda de los campos de norma teorías como el electromagnetismo, la electrodinámica cuántica, la gravitación, etcétera, con la ventaja de facilitar el cálculo y entender la importancia de la simetría grupo como algo más fundamental.

\subsection{Teoría de Norma No-Abeliana}

Comenzamos con una breve ejemplificación de algunos tópicos básicos que involucran a la teoría de norma [1]. La manera para describir la cosntrucción general de la teoría de norma no afecta del todo la física subyacente en ella salvo algunas excepciones. Como punto de partida se tomará la transformación de un grupo de simetría local arbitrario sobre una función escalar $\varphi(x)$ de prueba necesaria para observar el efecto que produce un campo de norma externo. Tenemos así la acción

$$
U \varphi(x)=\exp \left(-i a \varepsilon^{k}(x) F_{k}\right) \varphi(x),
$$

donde $a$ es una constante de acoplamiento, $\varepsilon^{k}(x)$ es el parámetro de transformación que depende de la posición y $F_{k}$ son los generadores de la simetría interna, con la siguiente relación de conmutación

$$
\left[F_{i}, F_{j}\right]=i c_{i j}^{k} F_{k}
$$

Bajo está simetría de transformación vamos a observar que tipo de conexión se deriva del campo escalar de prueba $\varphi(x)$. Para esto vamos a definir a la función $\varphi(x)$ como una combinación lineal de vectores de la siguiente forma

$$
\varphi(x)=\sum_{\alpha} \varphi_{\alpha}(x) u_{\alpha}
$$

donde $u_{\alpha}$ es un conjunto de vectores en el espacio interno equivalente a los componentes del spin isótopico y $\varphi_{\alpha}(x)$ son los componentes de $\varphi(x)$ en la base de $u_{\alpha}$. La utilidad de descomponer a $\varphi(x)$ en los componentes de una base $u_{\alpha}$ es observar el efecto que genera un potencial de campo externo sobre $\varphi(x)$ derivado de la base interna $u_{\alpha}$. 
Si la partícula $\varphi(x)$ experimenta un desplazamiento de $x$ a $x+d x$ la diferencia de cambio de $\varphi(x)$ es

$$
d \varphi(x)=\varphi(x+d x)-\varphi(x),
$$

por lo que ahora tenemos como propósito observar que sucede si aplicamos esté desplazamiento sobre (2.2), expandiendo a primer orden en $d x$ tenemos que

$$
d \varphi(x)=\sum_{\alpha}\left[\left(\partial_{\mu} \varphi_{\alpha}\right) d x^{\mu} u_{\alpha}+\varphi_{\alpha} d u_{\alpha}\right] .
$$

Como podemos observar el segundo término contiene el cambio $d u_{\alpha}$ para el espacio interno, que describirá el efecto que produce el potencial de campo externo sobre el espacio interno del campo escalar.

Por otra parte la forma de la transformacion infinitesimal para $U$ es

$$
\begin{aligned}
U(d x) & =\exp \left(-i a d \varepsilon^{k} F_{k}\right), \\
& =\exp \left(-i a\left(\partial_{\mu} \varepsilon^{k}\right) d x^{\mu} F_{k}\right) .
\end{aligned}
$$

Si realizamos una transformación sobre el espacio interno $u$ vamos a experimentar una cantidad de cambio para $u$ dada por

$$
U(d x) u=u+d u .
$$

Para el caso de la base de vectores $u_{\alpha}$ los generadores $F_{k}$ actúan como una matríz de operadores para la base $u_{\alpha}$, por lo que

$$
U(d x) u_{\alpha}=\exp \left[-a\left(\partial_{\mu} \varepsilon^{k}\right) d x^{\mu}\left(F_{k}\right)_{\alpha \beta}\right] u_{\beta} .
$$

Igualando las relaciones $(2.6),(2.7)$ y expandiendo a primer orden en $d x$, tenemos que

$$
u_{\alpha}+d u_{\alpha}=\left[\delta_{\alpha \beta}-i a\left(\partial_{\mu} \varepsilon^{k}\right) d x^{\mu}\left(F_{k}\right)_{\alpha \beta}\right] u_{\beta} .
$$

De la relación anterior (2.8) es fácil observar que el cambio en la base es

$$
d u_{\alpha}=-i a\left(\partial_{\mu} \varepsilon^{k}\right) d x^{\mu}\left(F_{k}\right)_{\alpha \beta} u_{\beta} .
$$

Ahora que tenemos el cambio $d u_{\alpha}$ podemos ver el efecto que produce el potencial de campo externo sobre $u_{\alpha}$, el cuál vamos a relacionar como la conexión de una transformación $U$ sobre el campo escalar $\varphi(x)$; para simplificar términos definiremos esta nueva conexión como

$$
\left(A_{\mu}\right)_{\alpha \beta}:=\left(\partial_{\mu} \varepsilon^{k}\right)\left(F_{k}\right)_{\alpha \beta} .
$$

De la ecuación (2.4) y observando la forma de las ecuaciones (2.9) y (2.10) obtenemos la forma final del cambio total

$$
d \varphi=\sum_{\alpha \beta}\left[\left(\partial_{\mu} \varphi_{\alpha}\right) \delta_{\alpha \beta}-i a\left(A_{\mu}\right)_{\alpha \beta} \varphi_{\alpha}\right] d x^{\mu} u_{\beta} .
$$


Observando la forma de la ecuación (2.11) es posible reacomodar los términos de la siguiente forma,

$$
\begin{aligned}
d \varphi & =\sum_{\beta}\left(d \varphi_{\beta}\right) u_{\beta} \\
& =\sum_{\beta}\left(D_{\mu} \varphi_{\beta}\right) d x^{\mu} u_{\beta} .
\end{aligned}
$$

Como se observa, $D_{\mu}$ es un nuevo operador que hace las veces de una derivada, mejor conocida como la derivada covariante, la cual describe el cambio que produce un potencial de campo externo sobre los componentes de $\varphi(x)$ en la base interna $u_{\alpha}$. De la ecuación (2.11) y (2.12) podemos ver que

$$
\begin{aligned}
D_{\mu} \varphi_{\beta} & =\sum_{\alpha}\left[\delta_{\alpha \beta} \partial_{\mu}-i a\left(A_{\mu}\right)_{\alpha \beta}\right] \varphi_{\alpha} \\
D_{\mu} & =\partial_{\mu}-i a A_{\mu} .
\end{aligned}
$$

Ahora que sabemos el efecto que produce un potencial de campo externo sobre un campo escalar de prueba $\varphi(x)$, podemos interpretar que $\left(A_{\mu}\right)_{\alpha \beta}$ es la norma que conecta al potencial de campo externo con la simetría interna del espacio de $\varphi(x)$.

\subsection{Teoría de Norma en Electromagnetismo}

La teoría electromagnética entendida de un manera simple [12] describe el efecto del campo magnético y eléctrico tanto en el vacío como al interactuar con cierto tipo de materiales, gobernadas por un conjunto de ecuaciones conocidas como las ecuaciones de Maxwell. Estas ecuaciones que resumen de manera efectiva el comportamiento de la teoría electromagnética, esconden entre sus ecuaciones una teoría de norma. Recordemos que las ecuaciones de Maxwell con fuentes son

$$
\begin{aligned}
\nabla \cdot D & =4 \pi \rho \\
\nabla \cdot B & =0 \\
\nabla \times H & =\frac{4 \pi}{c} J+\frac{1}{c} \frac{\partial D}{\partial t}, \\
\nabla \times E+\frac{1}{c} \frac{\partial B}{\partial t} & =0 .
\end{aligned}
$$

Para observar la teoría de norma subyacente en la teoría electromagnética, vamos a ver que relación existe entre los potenciales de campo vectorial $\vec{A}$ y escalar $\phi$ (ingredientes necesarios para definir a el campo de norma $A_{\mu}$ ) y los campos eléctrico y magnético a partir de las 
ecuaciones de Maxwell. Empezaremos con la siguiente ecuación de Maxwell $\nabla \cdot B=0$, la cual nos lleva a definir al campo magnético $B$ como el rotacional de un campo vectorial,

$$
B=\nabla \times A .
$$

Si sustituimos esta relación en la ecuación de Maxwell que esta relacionada con la ley de Faraday tenemos que

$$
\nabla \times\left(E+\frac{1}{c} \frac{\partial A}{\partial t}\right)=0 .
$$

Es fácil ver que si el rotacional de un campo vectorial es igual a cero, éste se pude relacionar con el gradiente de un campo escalar

$$
\begin{aligned}
E+\frac{1}{c} \frac{\partial A}{\partial t} & =-\nabla \phi, \\
E & =-\frac{1}{c} \frac{\partial A}{\partial t}-\nabla \phi .
\end{aligned}
$$

De esta forma las ecuaciones (2.15) y (2.16) nos muestran la relación que guardan los campos eléctrico y magnético con respecto a los nuevos potenciales de campo $\vec{A}$ y $\phi$. Para los potenciales de campo $A$ y $\phi$ se puede hacer la siguiente transformación

$$
\begin{gathered}
A^{\prime}=A+\nabla \lambda, \\
\phi^{\prime}=\phi-\frac{1}{c} \frac{\partial}{\partial t} \lambda .
\end{gathered}
$$

Esta transformación no afecta a las relaciones (2.15) y (2.16), lo cual nos dice que permanecen invariantes ante las transformaciones (2.17) y (2.18) llamadas transformaciones de norma ${ }^{1}$, siendo $\lambda$ una función escalar. El argumento anterior evidencia que se tiene la libertad para escoger un conjunto de potenciales $A$ y $\phi$ como mejor convenga.

Ya vimos que forma adoptó la ecuación de Maxwell que relaciona a la Ley de Faraday, pero aún falta ver que forma adquieren las dos ecuaciones de Maxwell que involucran la Ley de Gauss y la Ley de Ampere. Sustituyendo (2.15) y (2.16) tenemos lo siguiente

$$
\begin{gathered}
\nabla^{2} \phi+\frac{1}{c} \frac{\partial}{\partial t}(\nabla \cdot A)=-4 \pi \rho \\
\nabla^{2} A-\frac{1}{c^{2}} \frac{\partial^{2} A}{\partial t^{2}}-\nabla\left(\nabla \cdot A+\frac{1}{c} \frac{\partial \phi}{\partial t}\right)=-\frac{4 \pi}{c} J .
\end{gathered}
$$

Anteriormente habíamos comentado que se tiene la libertad de escoger los potenciales de campo $A$ y $\phi$; con esta consideración vamos a imponer la siguiente condición de manera conveniente

\footnotetext{
${ }^{1}$ la invarianza de los campos bajo estas transformaciones se conoce como invarianza de norma
} 


$$
\nabla \cdot A+\frac{1}{c} \frac{\partial \phi}{\partial t}=0
$$

A esta relación se le conoce como la condición de Lorentz y gracias a esta las ecuaciones (2.19) y (2.20) quedan como dos ecuaciones de onda inhomogeneas para $A$ y otra para $\phi$ :

$$
\begin{aligned}
\nabla^{2} \phi-\frac{1}{c^{2}} \frac{\partial^{2} \phi}{\partial t^{2}} & =-4 \pi \rho, \\
\nabla^{2} A-\frac{1}{c^{2}} \frac{\partial^{2} A}{\partial^{2} t} & =-\frac{4 \pi}{c} J .
\end{aligned}
$$

Para observar la conexión de las ecuaciones de Maxwell con la teoría de norma se define el siguiente cuadri-vector potencial

$$
A_{\mu}=(\phi, A) .
$$

En la teoría de relatividad especial, $A_{\mu}$ hace la función de nuestro campo de norma que surgió de la invarianza de los campos $\phi$ y $A$. Por lo tanto, a partir de nuestro campo de norma $A_{\mu}$ podemos obtener la dinámica de la teoría electromagnética siguiendo los pasos de la teoría de norma.

Si consideramos la derivada covariante (2.13) y calculamos $\left[D_{\mu}, D_{\nu}\right]$ vemos que:

$$
\begin{aligned}
{\left[D_{\mu}, D_{\nu}\right] \varphi=} & {\left[\partial_{\mu}+A_{\mu}, \partial_{\nu}+A_{\nu}\right] \varphi } \\
= & \left(\partial_{\mu}+A_{\mu}\right)\left(\partial_{\nu} \varphi+A_{\nu} \varphi\right)-\left(\partial_{\nu}+A_{\nu}\right)\left(\partial_{\mu} \varphi+A_{\mu} \varphi\right) \\
= & \partial_{\mu}\left(\partial_{\nu} \varphi\right)+\partial_{\mu}\left(A_{\nu} \varphi\right)+A_{\mu} \partial_{\nu} \varphi+A_{\mu} A_{\nu} \varphi \\
& -\partial_{\nu}\left(\partial_{\mu} \varphi\right)-\partial_{\nu}\left(A_{\mu} \varphi\right)-A_{\nu} \partial_{\mu} \varphi-A_{\nu} A_{\mu} \varphi
\end{aligned}
$$

Viendo la forma de el potencial de norma $A_{\mu}=(\phi, \vec{A})$ nos damos cuenta que el grupo de simetría interna es $U(1)$. Por lo tanto el conmutador $\left[A_{\mu}, A_{\nu}\right] \phi=0$, ya que estamos ante la presencia de una taoría abeliana, y el cálculo anterior queda como

$$
\begin{aligned}
{\left[D_{\mu}, D_{\nu}\right] \varphi=} & \partial_{\mu} A_{\nu} \varphi+A_{\nu} \partial_{\mu} \varphi+A_{\mu} \partial_{\nu} \varphi \\
& -\partial_{\nu} A_{\mu} \varphi-A_{\mu} \partial_{\nu} \varphi-A_{\nu} \partial_{\mu} \varphi \\
= & \left(\partial_{\mu} A_{\nu}-\partial_{\nu} A_{\mu}\right) \varphi .
\end{aligned}
$$

Del cálculo anterior podemos obtener un nuevo objeto llamado tensor de esfuerzos de Maxwell constituido como

$$
F_{\mu \nu}:=\left(\partial_{\mu} A_{\nu}-\partial_{\nu} A_{\mu}\right) .
$$

Es evidente que la teoría electromagnética es una teoría de norma abeliana que involucra cantidades y relaciones que permanecen invariantes ante las transformaciones (2.17) y (2.18), el descubrir a la teoría de norma de la teoría electromagnética es una evidencia clara que existe un principio básico en las simetrías de un sistema en general. 


\subsection{Teoría de Norma en Gravitación}

Una de las interrogantes más extensas en las ciencias físicas ha sido la unificación de diversas teorías en una sola más fundamental, y así obtener una estructura más completa del universo, como una explicación del todo. Esto ha derivado en varias propuestas para la unificación de teorías como la interacción débil y electromagnética que son útiles en el análisis de la interacción de partículas.

En base de la idea anteriormente esgrimida, es posible realizar la construcción desde el punto de vista de algún tipo de geometría, que sea capaz de contener a los elementos del espacio-tiempo y las simetrías de algún grupo local; esta idea se propone en el entendido que la invarianza de norma local se define sobre el espacio Minkowkiano que, sobre ciertos conceptos del mismo, tiene como consecuencia a la Relatividad General y la interacción de partículas.

Por lo tanto se definirá una nueva geometría expandida con elementos coordenados para el espacio-tiempo y los asociados a algún grupo de simetría local. A este super espacio lo conocemos como haz fibrado, el cual se define en dos sectores, uno horizontal (relacionado con el espacio-tiempo) y el otro vertical (relacionado con algún grupo de simetría local).

Tomaremos a $g_{\mu \nu}(x)$ como los componentes del tensor métrico del espacio 4-dimensional y a $g_{A B}(\theta)$ como los componentes del tensor métrico del grupo n-dimensional asociado a alguna simetría local de grupo; con esto construimos el espacio $(n+4)$-dimensional que tiene como tensor métrico el superespacio a $G_{i j}(y)$, y como coordenadas a los elementos

$$
y^{i}=\left\{x^{\mu}, \theta^{A}\right\},
$$

donde el indice $\mu=0, \ldots, 3, \mathrm{y} A=1, \ldots, n$. Para este superespacio es posible utilizar la estructura de la geometría diferencial ya que se define a $G_{i j}$ como la geometría del superespacio $y^{i}$; por lo tanto definimos la siguiente acción simétrica ${ }^{2}$

$$
\mathbf{G}(\mathbf{X}, \mathbf{Y})=G_{i j} e^{i} \otimes e^{j}(\mathbf{X}, \mathbf{Y}) .
$$

Entonces los elementos de $\mathbf{G}$ son

$$
\mathbf{G}=G_{i j} e^{i} \otimes e^{j}
$$

El tensor $\mathbf{G}$ al ser simétrico nos otorga la libertad de igualarlo a la línea de mundo $d S^{2}$

$$
d S^{2}=G_{i j} e^{i} e^{j}
$$

Los elementos de $G_{i j}$ es posible obtenerlos con la ayuda de las cantidades

$$
h_{i}{ }^{\mu}=\frac{\partial x^{\mu}}{\partial y^{i}}
$$

que son vectores que relacionan la dependencia del espacio-tiempo con el superespacio. A su vez definimos vectores normales $N_{A}^{i}$ al sector horizontal tal que

$$
h_{i}{ }^{\mu} N_{A}^{i}=0 .
$$

\footnotetext{
${ }^{2}$ La acción es simétrica para $\mathbf{G}(\mathbf{X}, \mathbf{Y})$ si $\mathbf{G}(\mathbf{X}, \mathbf{Y})=\mathbf{G}(\mathbf{Y}, \mathbf{X})$.
} 
Esto sugiere la existencia de una relación entre los componentes ${ }^{3} G_{i j}$ y $g_{\mu \nu}$, dada por la proyección del superespacio en el espacio-tiempo

$$
\begin{aligned}
g_{\mu \nu} & =h^{i}{ }_{\mu} h^{j}{ }_{\nu} G_{i j}, \\
g^{\mu \nu} & =h_{i}{ }^{\mu} h_{j}{ }^{\nu} G^{i j}
\end{aligned}
$$

y por lo tanto para $g_{A B}$ será

$$
\begin{aligned}
g_{A B} & =N^{i}{ }_{A} N^{j}{ }_{B} G_{i j}, \\
g^{A B} & =N_{i}{ }^{A} N_{j}{ }^{B} G^{i j} .
\end{aligned}
$$

Como en general el espacio-tiempo no depende de el grupo de simétrias tenemos que $\frac{\partial x^{\mu}}{\partial y^{A}}=0$; si en general $h_{A}^{\mu}$ es cero es posible conocer los elementos para $G_{i j}$ y su inversa mediante las expresiones

$$
\begin{aligned}
G_{i j} & =\left(\begin{array}{cc}
g_{\mu \nu}+g_{A B} N_{\mu}^{A} N_{\nu}^{B} & g_{A B} N_{\mu}^{A} \\
N_{\nu}{ }^{B} g_{A B}, & g_{A B}
\end{array}\right), \\
G^{i j} & =\left(\begin{array}{cc}
g^{\mu \nu}, & -g^{\mu \nu} N_{\nu}^{B} \\
-N_{\mu}^{A} g^{\mu \nu} & g^{A B}+N_{\mu}^{A} N_{\nu}^{B} g^{\mu \nu}
\end{array}\right) .
\end{aligned}
$$

A partir de la forma de $G_{i j}$ es posible determinar la línea de mundo y por ende obtener el escalar de curvatura para el superespacio $y^{i}$. Tenmos así que

$$
\begin{gathered}
d S^{2}=g_{\mu \nu} d x^{\mu} d x^{\nu}+g_{A B} d \theta^{A} d \theta^{B}+g_{A B} N_{\mu}{ }^{A} N_{\nu}{ }^{B} d x^{\mu} d x^{\nu}, \\
R=R_{e t}+R_{G}-\frac{1}{4} F_{\mu \nu}^{A} F_{A}^{\mu \nu},
\end{gathered}
$$

donde $R_{e t}$ es el escalar de curvatura del espacio-tiempo y $R_{G}$ el escalar de curvatura del grupo de simertría interna. A partir del escalar de curvatura del superespacio es posible obtener una estructura que unifique, a la teoría de la relatividad con la electrodinámica, o la teoría cuántica de campos, mediante una estructura geométrica extendida como un haz fibrado. En este trabajo solo se trabajará con la parte geometrica del espacio-tiempo que da sentido a un espacio curvado por la acción de campos gravitacionales, pero lo anterior queda como una generalización del tratamiento de la teoría de norma local como una aproximación geométrica [13] que puede ser útil en la construcción de teorías de unificación.

\subsection{Elementos de la Geometría Diferencial}

La geometría diferencial es una herramienta matemática usada para la teoría gravitacional [14] que es usada para obtener una descripción más sencilla del espacio-tiempo que se ve afectada por un campo gravitacional. A continuación se expondrá de forma breve los elementos básicos que componen a la geometría diferencial y de los cuales se hará uso más adelante.

\footnotetext{
${ }^{3}$ Los vectores inversos se definen como: $h_{i}{ }^{\mu} h^{i}{ }_{\nu}=\delta^{\mu}{ }_{\nu}, N_{i}{ }^{A} N^{i}{ }_{B}=\delta^{A}{ }_{B}$.
} 


\section{Vector Tangente y Cotangente}

Vamos a considerar una función ${ }^{4} f$ sobre alguna variedad $M$ que va de $\mathbb{R}^{n}$ a $\mathbb{R}$. Consideremos también una curva $\phi(t)$ sobre la variedad $M$; por lo tanto podemos relacionar los puntos de esta curva $\phi$ con la función $f$ de la siguiente forma $f(\rho(t))=f\left(x^{1}(t), \ldots, x^{n}(t)\right)$.

Tomando en cuenta lo anterior tenemos que

$$
\left(\frac{\partial f}{\partial t}\right)_{\phi\left(t_{0}\right)}=\left(\frac{d x^{i}}{d t} \frac{\partial f}{\partial x^{i}}\right)_{t=t_{0}} .
$$

Si tomamos en cuenta que existen una infinidad de curvas $\phi(t)$ que pasan a través de un punto $p$, se puede definir un espacio vectorial en el punto $p$ como una combinación lineal de los elementos $\partial / \partial x^{j}$ como sigue

$$
\mathbf{X}=X^{a} \frac{\partial}{\partial x^{a}} .
$$

A este espacio vectorial le llamaremos espacio vectorial tangencial al punto $p$ o simplemente $T_{p}$. Los elementos $\partial / \partial x^{j}$ constituyen vectores tangente en el punto $p$.

Es posible escoger una base arbitraria de vectores linealmente independientes en lugar de una base determinada por coordenadas locales a través de una relación lineal de transformación de la forma

$$
e_{a}=e_{a}{ }^{\mu} \frac{\partial}{\partial x^{\mu}} .
$$

A partir de (2.34) es sencillo ver que la relación inversa está dada por

$$
\frac{\partial}{\partial x^{\mu}}=e^{a}{ }_{\mu} e_{a} .
$$

$e_{a}{ }^{\mu}$ son los elementos inversos a $e^{a}{ }_{\mu}$ por lo que se cumple

$$
\begin{aligned}
& e_{a}{ }^{\mu} e^{a}{ }_{\nu}=\delta^{\mu}{ }_{\nu}, \\
& e_{a}{ }^{\mu} e^{b}{ }_{\mu}=\delta_{a}{ }^{b} .
\end{aligned}
$$

Entonces podemos expresar cualquier vector tangente al punto $p$ en la nueva base $e_{a}$

$$
\mathbf{X}=\tilde{X}^{a} e_{a} .
$$

donde los elementos $\tilde{X}^{a}$ se obtienen a partir de los componentes $X^{a}$ en (2.33). Por otra parte el espacio cotangente, también llamado la uno forma $\boldsymbol{\omega}$ al punto $p$, es una acción lineal del espacio tangencial a los reales

$$
\boldsymbol{\omega}: T_{p} \longrightarrow \mathbb{R}^{1} .
$$

Esto lo que nos quiere decir es, que tomando algún vector tangencial $\mathbf{X}$, la uno forma $\boldsymbol{\omega}$ esta asociada unicamente a un número $\boldsymbol{\omega}(\mathbf{X})$ dado por

\footnotetext{
${ }^{4}$ Definimos a $f$ como una función suave sobre las coordenadas $\left(x^{1}, \ldots, x^{n}\right)$
} 


$$
\boldsymbol{\omega}(\mathbf{X})=\langle\boldsymbol{\omega}, \mathbf{X}\rangle \text {. }
$$

A este espacio lo vamos a denotar como el espacio cotengente $T_{p}^{*}$ al punto $p$ sobre la variedad $M$, visto como el espacio dual al espacio tangente. La base para $T_{p}^{*}$ la asignamos como una base linealmente independiente arbitraria $e^{a}$, con lo cual es posible obtener los elementos del vector tangente $\mathbf{X}$

$$
\begin{aligned}
e^{a}(\mathbf{X}) & =\left\langle e^{a}, X^{b} e_{b}\right\rangle \\
& =\left\langle e^{a}, e_{b}\right\rangle X^{b} \\
& =\delta^{a}{ }_{b} X^{b} \\
& =X^{a} .
\end{aligned}
$$

Al utilizar $e^{a}\left(e_{b}\right)=\left\langle e^{a}, e_{b}\right\rangle=\delta^{a}{ }_{b}$, estamos diciendo que ambos son espacios duales entre sí.

Retomando la relación (2.36), se observa que los elementos de la uno-forma $\boldsymbol{\omega}$ se pueden escribir como

$$
\begin{aligned}
\boldsymbol{\omega}(\mathbf{X}) & =\langle\boldsymbol{\omega}, \mathbf{X}\rangle \\
& =\left\langle\boldsymbol{\omega}, X^{a} e_{a}\right\rangle \\
& =X^{a}\left\langle\boldsymbol{\omega}, e_{a}\right\rangle
\end{aligned}
$$

De lo anterior tenemos que

$$
\begin{aligned}
\boldsymbol{\omega}\left(e_{a}\right) & =\left\langle\boldsymbol{\omega}, e_{a}\right\rangle \\
& =\omega_{a} .
\end{aligned}
$$

Con los elementos de $\boldsymbol{\omega}$ en la base de $e_{a}$, y retomando el cálculo de (2.37) obtenemos lo siguiente

$$
\begin{aligned}
\langle\boldsymbol{\omega}, \mathbf{X}\rangle & =X^{a}\left\langle\boldsymbol{\omega}, e_{a}\right\rangle \\
& =\omega_{a} X^{a} \\
& =\omega_{a}\left\langle e^{a}, X^{b} e_{b}\right\rangle \\
& =\left\langle\omega_{a} e^{a}, \mathbf{X}\right\rangle .
\end{aligned}
$$

De lo anterior es fácil observar que la uno-forma $\boldsymbol{\omega}$ del espacio cotengente $T_{p}^{*}$ es una combinación lineal de la base de vectores cotangentes $e^{a}$

$$
\boldsymbol{\omega}=\omega_{a} e^{a} .
$$


Las bases duales $e_{a} \mathrm{y} e^{b}$ de los espacio tangente y cotangente al punto $p$ respectivamente, tienen la posibilidad de escoger una base diferente. En general tenemos

$$
\begin{aligned}
& e_{a^{\prime}}=\Phi_{a^{\prime}}{ }^{b} e_{b}, \\
& e^{a^{\prime}}=\Phi^{a^{\prime}}{ }_{b} e^{b},
\end{aligned}
$$

y al tener bases duales requerimos que tengan el siguiente comportamiento

$$
\begin{aligned}
\delta^{b^{\prime}}{ }_{a^{\prime}} & =\left\langle e^{b^{\prime}}, e_{a^{\prime}}\right\rangle \\
& =\Phi^{b^{\prime}}{ }_{b} \Phi_{a^{\prime}}{ }^{a}\left\langle e^{b}, e_{a}\right\rangle \\
& =\Phi^{b^{\prime}}{ }_{{ }^{\prime}} \Phi_{a^{\prime}}{ }^{a} \delta^{b}{ }_{a} \\
& =\Phi^{b^{\prime}}{ }_{b} \Phi_{a^{\prime}}{ }^{b} .
\end{aligned}
$$

Es evidente que las matrices de transformación $\Phi^{b^{\prime}}{ }_{b}$ y $\Phi_{a^{\prime}}{ }^{a}$ son inversas entre sí. Por lo tanto es facíl observar que un cambio de base en los elementos del vector tangente $\mathbf{X}$ y cotangente $\boldsymbol{\omega}$ se encuentra determinado por las relaciones

$$
\begin{aligned}
X^{a^{\prime}} & =e^{a^{\prime}}(\mathbf{X}) \\
& =\Phi^{a^{\prime}}{ }_{b} e^{b}(\mathbf{X}) \\
& =\Phi^{a^{\prime}}{ }_{b} X^{b}, \\
\omega_{a^{\prime}} & =\boldsymbol{\omega}\left(e_{a^{\prime}}\right) \\
& =\Phi_{a^{\prime}}{ }^{b} \boldsymbol{\omega}\left(e_{b}\right) \\
& =\Phi_{a^{\prime}}{ }^{b} \omega_{b} .
\end{aligned}
$$

Por último, debemos de mencionar que la transformación de una base coordenada $x^{a}$ a otra $x^{a^{\prime}}$ es posible a través de las matrices de transformación $\Phi^{b^{\prime}}{ }_{b}$ y $\Phi_{a^{\prime}}{ }^{a}$, las cuales guardan la siguiente relación Jacobiana

$$
\begin{aligned}
\Phi_{b}^{b^{\prime}} & =\left(\frac{\partial x^{b^{\prime}}}{\partial x^{b}}\right)_{p}, \\
\Phi_{a^{\prime}}{ }^{a} & =\left(\frac{\partial x^{a}}{\partial x^{a^{\prime}}}\right)_{p} .
\end{aligned}
$$

\section{Derivada Exterior}

Antes discutir la acción de la derivada exterior, es necesario considerar la siguiente acción multilineal 


$$
\mathbf{T}: \Pi_{s}^{r} \rightarrow \mathbb{R}^{1}
$$

donde $\Pi_{s}^{r}$ es el espacio de tensores del tipo $(r, s)$.

Este acción proporciona una asociasión de un ordenamiento de $r$ uno-formas y $s$ vectores tangenciales a un número real

$$
\mathbf{T}\left(\boldsymbol{\omega}^{1}, \ldots, \boldsymbol{\omega}^{r}, \mathbf{X}_{1}, \ldots, \mathbf{X}_{s}\right)=a .
$$

A partir de la acción anterior es posible determinar cuales son los elementos de $T_{s}^{r}$

$$
\begin{aligned}
\mathbf{T}\left(\boldsymbol{\omega}^{1}, \ldots, \boldsymbol{\omega}^{r}, \mathbf{X}_{1}, \ldots, \mathbf{X}_{s}\right) & =\mathbf{T}\left(\omega^{1}{ }_{a_{1}} e^{a_{1}}, \ldots, \omega^{r}{ }_{a_{r}} e^{a_{r}}, X_{1}{ }^{b_{1}} e_{b_{1}}, \ldots, X_{s}{ }^{b_{s}} e_{b_{s}}\right) \\
& =\omega^{1}{ }_{a_{1}} \ldots \omega^{r}{ }_{a_{r}} X_{1}{ }^{b_{1}} \ldots X_{s}{ }^{b_{s}} \mathbf{T}\left(e^{a_{1}}, \ldots, e^{a_{r}}, e_{b_{1}}, \ldots, e_{a_{s}}\right)
\end{aligned}
$$

De este reacomodo de términos obtenemos los elementos de $\mathbf{T}$ relativos a una base construida mediante las bases $e^{a}$ y $e_{a}$. Por medio de ellas obtenemos los componentes

$$
\mathbf{T}\left(e^{a_{1}}, \ldots, e^{a_{r}}, e_{b_{1}}, \ldots, e_{b_{s}}\right)=T_{b_{1} \ldots b_{s}}^{a_{1} \ldots a_{r}} .
$$

Por lo tanto tenemos que

$$
\mathbf{T}=T_{b_{1} \ldots b_{s}}^{a_{1} \ldots a_{a_{r}}} e^{a_{1}} \otimes \ldots \otimes e^{a_{r}} \otimes e_{b_{1}} \otimes \ldots \otimes e_{b_{s}}
$$

Como es posible observar de la ecuación (2.42), los componentes de $\mathbf{T}$ en la base dual $e_{a}$ y $e^{b}$ son la representación de productos tensoriales. Bajo esta notación es posible tomar el siguiente producto tensorial

$$
\mathbf{Y}_{1} \otimes \ldots \otimes \mathbf{Y}_{s} \otimes \mathbf{\Omega}^{1} \otimes \ldots \otimes \mathbf{\Omega}^{r}
$$

Estos $r$ vectores tangentes y $s$ uno-formas son los elementos de $T_{s}^{r}$ que correlaciona a un número real de la siguiente forma

$$
\begin{aligned}
& \mathbf{Y}_{1} \otimes \ldots \otimes \mathbf{Y}_{s} \otimes \boldsymbol{\Omega}^{1} \otimes \ldots \otimes \boldsymbol{\Omega}^{r}\left(\boldsymbol{\omega}^{1}, \ldots, \boldsymbol{\omega}^{r}, \mathbf{X}_{1}, \ldots, \mathbf{X}_{s}\right) \\
= & \left\langle\boldsymbol{\omega}^{1}, \mathbf{Y}_{1}\right\rangle \ldots\left\langle\boldsymbol{\omega}^{r}, \mathbf{Y}_{r}\right\rangle\left\langle\boldsymbol{\Omega}^{1}, \mathbf{X}_{1}\right\rangle \ldots\left\langle\boldsymbol{\Omega}^{s}, \mathbf{X}_{s}\right\rangle .
\end{aligned}
$$

Ya que se expuso de manera breve la construcción de un tensor, ahora vamos a ver el caso particular de un tensor del tipo $(0, s)$ totalmente antisimétrico, lo que implica que los tensores covariantes son antisimétricos en cada par de sus argumentos

$$
\mathbf{T}\left(\mathbf{X}_{1}, \ldots, \mathbf{X}_{a}, \ldots, \mathbf{X}_{b}, \ldots, \mathbf{X}_{s}\right)=-\mathbf{T}\left(\mathbf{X}_{1}, \ldots, \mathbf{X}_{b}, \ldots, \mathbf{X}_{a}, \ldots, \mathbf{X}_{s}\right)
$$

Los tensores de este tipo son llamados s-formas, las componentes $T_{a_{1} \ldots a_{s}}$ se calculan en términos de la base relativa,

$$
e^{a_{1}} \otimes \ldots \otimes e^{a_{s}}
$$


Bajo este argumento de tener un tensor $(0, s)$ totalmente antisiémtrico, vamos a representar los elementos de la base de tensores antisimétricos como

$$
e^{a_{1}} \wedge \ldots \wedge e^{a_{s}}
$$

A este nuevo producto lo conocemos como producto exterior, por lo tanto de forma general podemos escribir la $s$-forma como

$$
\Omega=\Omega_{a_{1} \ldots a_{s}} e^{a_{1}} \wedge e^{a_{2}} \wedge \ldots \wedge e^{a_{s}} .
$$

Como es sencillo observar, al realizar un intercambio de índices, el producto exterior cambia de signo por la característica de antisimétria.

$$
e^{a} \wedge e^{b}=-e^{b} \wedge e^{a}
$$

Consideramos ahora la derivada total de una función $f$,

$$
d f=\frac{\partial f}{\partial x^{a}} d x^{a}
$$

Nos percatamos que $d f$ es una uno-forma en la base local de $d x^{a}$, lo cual nos dice que $d f=\alpha_{i} d x^{i}$, de modo que

$$
\begin{aligned}
d f(\mathbf{X}) & =\left\langle\alpha_{a} d x^{a}, X^{b} \frac{\partial}{\partial x^{b}}\right\rangle \\
& =\alpha_{a} X^{b}\left\langle d x^{a}, \frac{\partial}{\partial x^{b}}\right\rangle \\
& =\alpha_{a} X^{a}
\end{aligned}
$$

por lo tanto $\alpha_{a}$ es igual a $\frac{\partial f}{\partial x^{a}}=f_{, a}^{5}$, lo cual nos arroja la siguiente relación

$$
\begin{aligned}
d f(\mathbf{X}) & =X^{a} f_{, a} \\
& =\mathbf{X} f
\end{aligned}
$$

A partir de la definición de la uno forma $d f$, es posible ver que la acción de esta derivada a la cual llamaremos derivada exterior, convierte la $p$-forma a una $(p+1)$-forma, por lo tanto si la función $f$ es una cero forma, ésta es transformada a la uno-forma $d f$, el siguiente cálculo hace evidente la acción de la derivada exterior sobre la $s$-forma.

$$
\begin{aligned}
d \mathbf{A} & =d\left(A_{a_{1} \ldots a_{s}} d x^{a_{1}} \wedge \ldots \wedge d x^{a_{s}}\right) \\
& =d A_{a_{1} \ldots a_{s}} \wedge e^{a_{1}} \wedge \ldots \wedge e^{a_{s}} \\
& =\frac{\partial A_{a_{1} \ldots a_{s}}}{\partial x^{b}} d x^{b} \wedge d x^{a_{1}} \wedge \ldots \wedge d x^{a_{s}}
\end{aligned}
$$

\footnotetext{
${ }^{5}$ Se introduce la notación donde la coma como subíndice denota diferencia parcial $\frac{\partial \phi}{\partial x^{a}}=\phi_{, a}$
} 


\section{Corchete de Lie}

El corchete de Lie se define como la acción de dos vectores de campo $\mathbf{X}$ y $\mathbf{Y}$ sobre una función $f$ cualquiera de la siguiente forma

$$
\begin{aligned}
{[\mathbf{X}, \mathbf{Y}] f: } & =(\mathbf{X Y}-\mathbf{Y X}) f \\
& =\mathbf{X}(\mathbf{Y} f)-\mathbf{Y}(\mathbf{X} f) .
\end{aligned}
$$

Los componentes del corchete de Lie en la base local de coordenadas se obtiene de la siguiente forma

$$
\begin{aligned}
{[\mathbf{X}, \mathbf{Y}]^{a}: } & =(\mathbf{X Y}-\mathbf{Y X}) x^{a} \\
& =\mathbf{X} Y^{b} \frac{\partial}{\partial x^{b}} x^{a}-\mathbf{Y} X^{b} \frac{\partial}{\partial x^{b}} x^{a} \\
& =\mathbf{X} Y^{b} \delta_{b}{ }^{a}-\mathbf{Y} X^{b} \delta_{b}{ }^{a} \\
& =X^{c} Y_{, c}^{a}-Y^{c} X_{, c}^{a} .
\end{aligned}
$$

El corchete de Lie $[\mathbf{X}, \mathbf{Y}]$ tiene relación con la derivada de Lie de $\mathbf{Y}$ en la dirección de $\mathbf{X}$ teniéndose

$$
\mathscr{L}_{\mathbf{X}} \mathbf{Y}=[\mathbf{X}, \mathbf{Y}]
$$

Ya expuesta la derivada de Lie, a modo de ejemplificación vamos a ver que sucede al aplicar esta derivada sobre una función escalar $f$. Tenemos

$$
\mathscr{L}_{\mathbf{X}} f=\mathbf{X} f=d f(\mathbf{X}) .
$$

Para este caso vemos que la acción de $\mathscr{L}_{\mathbf{X}}$ sobre una función escalar, es la asociación de la uno forma $d f$ y el vector de campo tangencial $\mathbf{X}$. Mediante la relación de la derivada de Lie con el corchete de Lie es posible mostrar que

$$
\begin{gathered}
\mathscr{L}_{\mathbf{X}}(\mathbf{S} \otimes \mathbf{T})=\mathscr{L}_{\mathbf{X}} \mathbf{S} \otimes \mathbf{T}+\mathbf{S} \otimes \mathscr{L}_{\mathbf{X}} \mathbf{T} \\
\mathscr{L}_{\mathbf{X}}(\boldsymbol{\omega} \otimes \mathbf{Y})=\left(\mathscr{L}_{\mathbf{X}} \boldsymbol{\omega}\right) \otimes \mathbf{Y}+\boldsymbol{\omega} \otimes\left(\mathscr{L}_{\mathbf{X}} \mathbf{Y}\right)
\end{gathered}
$$

donde $\boldsymbol{S}, \boldsymbol{T}$ y $\boldsymbol{Y}$ son tensores, mientras que $\boldsymbol{\omega}$ es la uno forma diferencial.

En particular es interesante ver el desglose del siguiente cálculo

$$
\begin{aligned}
\mathscr{L}_{\mathbf{X}}\langle\boldsymbol{\omega}, \mathbf{Y}\rangle & =\left\langle\mathscr{L}_{\mathbf{X}} \boldsymbol{\omega}, \mathbf{Y}\right\rangle+\left\langle\boldsymbol{\omega}, \mathscr{L}_{\mathbf{X}} \mathbf{Y}\right\rangle \\
X^{a}\left(\omega_{b} Y^{b}\right)_{, a} & =\left(\mathscr{L}_{\mathbf{X}} \boldsymbol{\omega}\right)_{b} Y^{b}+\omega_{b}\left(\mathscr{L}_{\mathbf{X}} \mathbf{Y}\right)^{b}
\end{aligned}
$$

Tomando la relación de (2.48), es posible ver que

$$
\begin{aligned}
\left(\mathscr{L}_{\mathbf{X} \boldsymbol{\omega}}\right)_{a} Y^{a} & =X^{b}\left(\omega_{a, b} Y^{a}+\omega_{a} Y_{, b}^{a}\right)-\omega_{a}\left(X^{b} Y_{, b}^{a}-Y^{b} X_{, b}^{a}\right) \\
& =\left(X^{b} \omega_{a, b}+\omega_{b} X_{, a}^{b}\right) Y^{a} .
\end{aligned}
$$




\section{Derivada Covariante}

Anteriormente se había observado como se realiza la tranformación de un base coordenada a otra, lo cual nos indica si existe invarianza ante transformaciones. Para tener un mejor entendimiento vamos a observar como transforma la parcial de una función escalar $f$. Tenemos que

$$
\frac{\partial f}{\partial x^{a^{\prime}}}=\frac{\partial f}{\partial x^{a}} \frac{\partial x^{a}}{\partial x^{a^{\prime}}}
$$

Esto nos dice que la transformación es lineal, y que $\frac{\partial f}{\partial x^{a}}$ transforma como un vector covariante, por lo tanto la derivada parcial sobre una función escalar es independiente a la base local. Pero veamos que sucede cuando realizamos el mismo procedimiento para un vector covariante $\omega_{a^{\prime}}$

$$
\begin{aligned}
\omega_{a^{\prime}, b^{\prime}} & =\frac{\partial}{\partial x^{b^{\prime}}}\left(\frac{\partial x^{a}}{\partial x^{a \prime}} \omega_{a}\right) \\
& =\frac{\partial^{2} x^{a}}{\partial x^{b \prime} \partial x^{a \prime}} \omega_{a}+\frac{\partial x^{a}}{\partial x^{a \prime}} \frac{\partial x^{b}}{\partial x^{b^{\prime}}} \omega_{a, b} .
\end{aligned}
$$

Nos percatamos que la derivada parcial de un vector covariante no es invariante ante cambios de base, por este hecho es esencial buscar un operador que al actuar con un vector covariante sea invariante ante cambios de base. Este operador propuesto será $\nabla_{\mathbf{X}}$, el cual asigna un vector de campo sobre $M$ a un operador diferencial, que mapea a un vector de campo arbitrario $\mathbf{Y}$, a un vector de campo $\nabla_{\mathbf{X}} \mathbf{Y}$ concido como derivada covariante de $\mathbf{Y}$. Tomando en cuenta esto vamos a pedir las siguientes condiciones

1. $\nabla_{\mathbf{X}} \mathbf{Y}$ es lineal en el argumento $\mathbf{X}$

$$
\nabla_{f \mathbf{X}+g \mathbf{Y}} \mathbf{Z}=f \nabla_{\mathbf{X}} \mathbf{Z}+g \nabla_{\mathbf{Y}} \mathbf{Z}
$$

2. $\nabla_{\mathbf{X}} \mathbf{Y}$ lineal en el argumento de $\mathbf{Y}$

$$
\nabla_{\mathbf{X}}(\mathbf{Y}+\mathbf{Z})=\nabla_{\mathbf{X}} \mathbf{Y}+\nabla_{\mathbf{X}} \mathbf{Z}
$$

3. $\nabla_{\mathbf{X}} f=\mathbf{X} f$.

Para ver como se construye la conexión afín del operador $\nabla_{\mathbf{X}}$, vamos a realizar el cálculo de $\nabla_{\mathbf{X}} \mathbf{Y}$ en términos de alguna base dual $e_{a} \mathrm{y} e^{b}$. Tenemos que

$$
\begin{aligned}
\nabla_{\mathbf{X}} \mathbf{Y} & =\nabla_{\mathbf{X}}\left(Y^{a} e_{a}\right) \\
& =\left(\nabla_{\mathbf{X}} Y^{a}\right) e_{a}+Y^{a}\left(\nabla_{\mathbf{X}} e_{a}\right) \\
& =\left(\mathbf{X} Y^{a}\right) e_{a}+Y^{a} \nabla_{\mathbf{X}} e_{a}
\end{aligned}
$$

Si observamos que $\nabla_{\mathbf{X}} e_{a}$ es posible reescribirlo como $\nabla e_{a}(\mathbf{X})$, y partimos de que la accion del operador $\nabla$ sobre la base $e_{a}$ es un tensor del tipo $(1,1)$ en la base de $e_{a}$, podemos escribir 


$$
\begin{aligned}
\nabla_{\mathbf{X}} e_{a} & =\nabla e_{a}(\mathbf{X}) \\
& =\omega^{c}{ }_{a}(\mathbf{X}) e_{c} .
\end{aligned}
$$

Sustituyendo la relación (2.57) en (2.56) tenemos lo siguiente

$$
\begin{aligned}
\nabla_{\mathbf{X}} \mathbf{Y} & =\left(\mathbf{X} Y^{a}\right) e_{a}+Y^{a} \omega^{c}{ }_{a}(\mathbf{X}) e_{c} \\
& =\left[\mathbf{X} Y^{a}+\omega^{a}{ }_{b}(\mathbf{X}) Y^{b}\right] e_{a}
\end{aligned}
$$

Por lo tanto de relación anterior es posible observar que la componente $a$-ésima de $\nabla_{\mathbf{X}} \mathbf{Y}$ es

$$
\left(\nabla_{\mathbf{X}} \mathbf{Y}\right)^{a}=\mathbf{X} Y^{a}+\omega^{a}{ }_{b}(\mathbf{X}) Y^{b}
$$

Si descomponemos el operador derivada covariante de la siguiente forma,

$$
\begin{aligned}
\nabla_{X^{d} e_{d}} \mathbf{Y} & =\nabla \mathbf{Y}\left(X^{d} e_{d}\right) \\
& =X^{d} \nabla \mathbf{Y}\left(e_{d}\right) \\
& =X^{d} \nabla_{e_{d}} \mathbf{Y}
\end{aligned}
$$

entonces la relación (2.58) queda de la siguiente forma

$$
\begin{aligned}
X^{d}\left(\nabla_{e_{d}} \mathbf{Y}\right)^{a} & =X^{d} e_{d} Y^{a}+\omega^{a}{ }_{b}\left(e_{d}\right) X^{d} Y^{b} \\
& =X^{d}\left[e_{d} Y^{a}+\omega^{a}{ }_{b}\left(e_{d}\right) Y^{b}\right]
\end{aligned}
$$

De esta forma de la derivada covariante vemos un nuevo elemento que esta formado por el término que incluye a $\omega^{a}{ }_{b}\left(e_{d}\right)$, los cuales son los elementos de $\omega^{a}{ }_{b}$ en la base de $e_{d}$. Por lo tanto podemos escribir $\omega^{a}{ }_{b}\left(e_{d}\right)=\omega^{a}{ }_{b d}$.

Si consideramos la base local de coordenadas, la ecuación (2.58) será

$$
\left(\nabla_{\partial_{a}} \mathbf{Y}\right)^{b}=\partial_{a} Y^{b}+\omega_{c a}^{b} Y^{c}
$$

Si $\Omega$ es una uno-forma, entonces para cualquier vector de campo $\mathbf{Y}$ es posible cálcular la acción de la derivada covariante de la siguiente forma

$$
\nabla_{\mathbf{X}}(\boldsymbol{\Omega}(\mathbf{Y}))=\left(\nabla_{\mathbf{X}} \boldsymbol{\Omega}\right) \mathbf{Y}+\boldsymbol{\Omega}\left(\nabla_{\mathbf{X}} \mathbf{Y}\right)
$$

y en términos de una base local $e_{a} \mathrm{y} e_{b}$, tenemos la expresión

$$
\nabla_{\mathbf{X}}\left(\Omega_{a} Y^{a}\right)=\left(\nabla_{\mathbf{X}} \Omega\right)_{a} Y^{a}+\Omega_{a}\left(\nabla_{\mathbf{X}} \mathbf{Y}\right)^{a} .
$$

De esta última relación y con el uso de la relación (2.58), es posible determinar la derivada covariante de la uno-forma $\Omega$, 


$$
\begin{aligned}
\left(\nabla_{\mathbf{X}} \Omega\right)_{a} Y^{a} & =\mathbf{X}\left(\Omega_{a} Y^{a}\right)-\Omega_{a}\left(\mathbf{X} Y^{a}+\omega^{a}{ }_{b}(\mathbf{X}) Y^{b}\right) \\
& =\left(\mathbf{X} \Omega_{a}\right) Y^{a}+\Omega_{a}\left(\mathbf{X} Y^{a}\right)-\Omega_{a}\left(\mathbf{X} Y^{a}\right)-\Omega_{b} \omega^{b}{ }_{a}(\mathbf{X}) Y^{a} \\
& =\left(\mathbf{X} \Omega_{a}-\Omega_{b} \omega^{b}{ }_{a}(\mathbf{X})\right) Y^{a} .
\end{aligned}
$$

De aquí vemos que el elemento $a$-ésimo de la derivada covariante de la uno forma es

$$
\left(\nabla_{\mathbf{X}} \Omega\right)_{a}=\mathbf{X} \Omega_{a}-\Omega_{b} \omega^{b}{ }_{a}(\mathbf{X}) .
$$

Entonces la relación (2.60) en la base local de coordenadas queda

$$
\left(\nabla_{\partial_{a}} \Omega\right)_{b}=\partial_{a} \Omega_{b}-\omega^{c}{ }_{a b} \Omega_{c} .
$$

Como anterioremete se ha mencionado, la derivada covariante contiene la conexión que hace posible que la derivada de un vector covariante o contravariante sea invariante ante transformaciones de base.

\section{Ecuaciones de E. Cartan}

Una de las principales ecuaciones de Cartan es la que esta relacionada con el tensor de torsión. El tensor de torsión es la diferencia que existe entre la derivada covariante y la diferencial de Lie, y se define así para poder observar el cambio que produce el transporte de un campo vectorial sobre una trayectoria. La torsión la definimos como la acción

$$
\mathbf{T}(\mathbf{X}, \mathbf{Y})=\nabla_{\mathbf{X}} \mathbf{Y}-\nabla_{\mathbf{Y}} \mathbf{X}-[\mathbf{X}, \mathbf{Y}] .
$$

Si nos posicionamos en la base $\left(e_{a}\right)$ y $\left(e^{b}\right)$, es posible obtener la forma del tensor de torsión. De (2.61) cálculamos lo siguiente

$$
\begin{aligned}
\nabla_{\mathbf{X}} \mathbf{Y}-\nabla_{\mathbf{Y}} \mathbf{X} & =\mathbf{X} Y^{a} e_{a}+\omega^{a}{ }_{b}(\mathbf{X}) Y^{b} e_{a}-\mathbf{Y} X^{a} e_{a}-\omega^{a}{ }_{b}(\mathbf{Y}) X^{b} e_{a} \\
& =\left[\mathbf{X} e^{a}(\mathbf{Y})+\omega^{a}{ }_{b}(\mathbf{X}) e^{b}(\mathbf{Y})-\mathbf{Y} e^{a}(\mathbf{X})-\omega^{a}{ }_{b}(\mathbf{Y}) e^{b}(\mathbf{X})\right] e_{a} \\
& =\left[\mathbf{X}\left\langle e^{a}, \mathbf{Y}\right\rangle-\mathbf{Y}\left\langle e^{a}, \mathbf{X}\right\rangle+\omega^{a}{ }_{b}(\mathbf{X}) e^{b}(\mathbf{Y})-\omega^{a}{ }_{b}(\mathbf{Y}) e^{b}(\mathbf{X})\right] e_{a}
\end{aligned}
$$

Utilizando ahora el elemento $a$-ésimo del corchete de Lie dado por la relación

$$
\begin{aligned}
{[\mathbf{X}, \mathbf{Y}] } & =[\mathbf{X}, \mathbf{Y}]^{a} e_{a} \\
& =e^{a}([\mathbf{X}, \mathbf{Y}]) e_{a} \\
& =\left\langle e^{a},[\mathbf{X}, \mathbf{Y}]\right\rangle e_{a}
\end{aligned}
$$

vemos que si sustituimos las relaciones (2.62) y (2.63) en la relación de la acción de la torsión (2.61), tenemos 
$\mathbf{T}(\mathbf{X}, \mathbf{Y})=\left[\mathbf{X}\left\langle e^{a}, \mathbf{Y}\right\rangle-\mathbf{Y}\left\langle e^{a}, \mathbf{X}\right\rangle-\left\langle e^{a},[\mathbf{X}, \mathbf{Y}]\right\rangle+\omega^{a}{ }_{b}(\mathbf{X}) e^{b}(\mathbf{Y})-\omega^{a}{ }_{b}(\mathbf{Y}) e^{b}(\mathbf{X})\right] e_{a}$

Si cálculamos ahora la derivada exterior de la uno-forma $\boldsymbol{\omega}$ obtenemos

$$
\begin{aligned}
d \boldsymbol{\omega}(\mathbf{X}, \mathbf{Y}) & =\omega_{a, b} e^{b} \wedge e^{a}(\mathbf{X}, \mathbf{Y}) \\
& =\frac{1}{2} \omega_{a, b}\left(e^{b} \wedge e^{a}-e^{a} \wedge e^{b}\right)(\mathbf{X}, \mathbf{Y}) \\
& =\frac{1}{2} \omega_{a, b}\left(\left\langle e^{b}, \mathbf{X}\right\rangle\left\langle e^{a}, \mathbf{Y}\right\rangle-\left\langle e^{a}, \mathbf{X}\right\rangle\left\langle e^{b}, \mathbf{Y}\right\rangle\right) \\
& =\frac{1}{2}\left(\omega_{a, b} X^{b} Y^{a}-\omega_{a, b} X^{a} Y^{b}\right) \\
& =\frac{1}{2}\left(\omega_{b, a}-\omega_{a, b}\right) X^{a} Y^{b}
\end{aligned}
$$

Si agregamos y restamos el término $Y^{b} \omega_{a} X_{, b}^{a}$ a la relación (2.64) tendremos lo siguiente

$$
\begin{aligned}
d \boldsymbol{\omega}(\mathbf{X}, \mathbf{Y}) & =\frac{1}{2}\left[Y^{b}\left(\omega_{b, a} X^{a}+\omega_{a} X_{, b}^{a}\right)-Y^{b}\left(\omega_{a, b} X^{a}+\omega_{a} X_{, b}^{a}\right)\right] \\
& =\frac{1}{2}\left[\mathbf{X}\left(\omega_{b} Y^{b}\right)-\omega_{b} \mathbf{X} Y^{b}+\omega_{a} \mathbf{Y} X^{a}-\mathbf{Y}\left(\omega_{a} X^{a}\right)\right] \\
& =\frac{1}{2}(\mathbf{X}\langle\boldsymbol{\omega}, \mathbf{Y}\rangle-\mathbf{Y}\langle\boldsymbol{\omega}, \mathbf{X}\rangle-\langle\boldsymbol{\omega},[\mathbf{X}, \mathbf{Y}]\rangle) .
\end{aligned}
$$

Haciendo uso de la ecuación de la derivada exterior de la uno-forma $\boldsymbol{\omega}$, tenemos que la relación (2.64) queda como sigue

$$
\begin{aligned}
\mathbf{T}(\mathbf{X}, \mathbf{Y})= & {\left[\mathbf{X}\left\langle e^{a}, \mathbf{Y}\right\rangle-\mathbf{Y}\left\langle e^{a}, \mathbf{X}\right\rangle-\left\langle e^{a},[\mathbf{X}, \mathbf{Y}]\right\rangle\right.} \\
& \left.+\omega^{a}{ }_{b}(\mathbf{X}) e^{b}(\mathbf{Y})-\omega^{a}{ }_{b}(\mathbf{Y}) e^{b}(\mathbf{X})\right] e_{a} \\
= & 2\left(d e^{a}+\omega^{a}{ }_{b} \wedge e^{b}\right) e_{a}(\mathbf{X}, \mathbf{Y}) .
\end{aligned}
$$

Por lo tanto los elementos del tensor de torsión son

$$
\frac{1}{2} T^{a}=d e^{a}+\omega^{a}{ }_{b} \wedge e^{b} .
$$

La ecuación (2.66) es conocida como la primera ecuación de Cartan. Para el caso donde la torsión es cero, lo cual garantiza transporte paralelo, tenemos que la ecuación (2.66) se reduce a

$$
d e^{a}+\omega^{a}{ }_{b} \wedge e^{b}=0 .
$$

La segunda ecuación de Cartan se relaciona con la curvatura, la cual nos aporta la información de que tan intenso es el espacio gravitacional en cada punto. La segunda ecuación 
de Cartan se origina de la conexión afín que nos provee la derivada covariante, lo que nos permite definir al tensor de curvatura como

$$
\mathbf{R}(\mathbf{X}, \mathbf{Y}) \mathbf{Z}:=\nabla_{\mathbf{X}} \nabla_{\mathbf{Y}} \mathbf{Z}-\nabla_{\mathbf{Y}} \nabla_{\mathbf{X}} \mathbf{Z}-\nabla_{[\mathbf{X}, \mathbf{Y}]} \mathbf{Z} .
$$

La forma anterior nos proporciona la información de que tanto cambia un tensor al ser transportado de forma paralela, bajo la diferencia de la acción del conmutador de la derivada covariante en la dirección del vector de campo X y Y y la acción de la derivada covariante en la dirección del corchete de Lie. A continuación vamos a desglosar la forma de la relación (2.68).

Primero vamos a ver que resultado arroja $\nabla_{\mathbf{X}} \nabla_{\mathbf{Y}} \mathbf{Z}$. Para ello calculamos

$$
\begin{aligned}
\nabla_{\mathbf{X}} \nabla_{\mathbf{Y}} \mathbf{Z}= & \nabla_{\mathbf{X}}\left(\mathbf{Y} Z^{a}+\omega^{a}{ }_{b}(\mathbf{Y}) Z^{b}\right) e_{a} \\
= & \left(\mathbf{Y} Z^{a}+\omega^{a}{ }_{b}(\mathbf{Y}) Z^{b}\right) \nabla_{\mathbf{X}} e_{a}+\mathbf{X Y} e^{a}(\mathbf{Z}) e_{a} \\
& +e^{b}(\mathbf{Z}) \mathbf{X} \omega^{a}{ }_{b}(\mathbf{Y}) e_{a}+\omega^{a}{ }_{b}(\mathbf{Y}) \mathbf{X} e^{b}(\mathbf{Z}) e_{a} .
\end{aligned}
$$

Ahora veamos como es la derivada covariante de $\mathbf{Z}$ en la dirección del corchete de Lie. Tenemos

$$
\nabla_{[\mathbf{X}, \mathbf{Y}]}=\left\{[\mathbf{X}, \mathbf{Y}] e^{a}(\mathbf{Z})+\omega^{a}{ }_{b}([\mathbf{X}, \mathbf{Y}]) e^{b}(\mathbf{Z})\right\} e_{a}
$$

Haciendo uso de los resultados de (2.69) y (2.70), vemos que la acción de la curvatura (2.68) queda como

$$
\begin{aligned}
\mathbf{R}(\mathbf{X}, \mathbf{Y}) \mathbf{Z}= & {\left[\mathbf{X}\left\langle\omega^{a}{ }_{b}, \mathbf{Y}\right\rangle-\mathbf{Y}\left\langle\omega^{a}{ }_{b}, \mathbf{X}\right\rangle-\left\langle\omega^{a}{ }_{b},[\mathbf{X}, \mathbf{Y}]\right\rangle\right.} \\
& \left.+\omega^{a}{ }_{c}(\mathbf{X}) \omega^{c}{ }_{b}(\mathbf{Y})-\omega^{a}{ }_{c}(\mathbf{Y}) \omega^{c}{ }_{b}(\mathbf{X})\right] e^{b}(\mathbf{Z}) e_{a} \\
= & 2\left(d \omega^{a}{ }_{b}+\omega^{a}{ }_{c} \wedge \omega^{c}{ }_{b}\right)(\mathbf{X}, \mathbf{Y}) e^{b}(\mathbf{Z}) e_{a} .
\end{aligned}
$$

A partir de esta expresión tenemos que la segunda ecuación de Cartan es

$$
\frac{1}{2} R_{b c d}^{a} e^{c} \wedge e^{d}=d \omega_{b}^{a}+\omega^{a}{ }_{c} \wedge \omega^{c}{ }_{b} .
$$

De esta forma se observa que la segunda ecuación de Cartan está relacionada con el tensor de Riemann con la derivada exterior de la uno forma $\omega^{a}{ }_{b}$.

\section{La métrica en la base de vectores cotangente}

Como sabemos la información de la curvatura del espacio-tiempo está contenida en la métrica $g_{\mu \nu}$, con la que podemos cálcular el elemento de línea; a este elemento de línea lo podemos definir como una correlación lineal de un tensor simétrico del tipo $(0,2)$. Los elementos para este tensor en una base local $e^{a}$ son coeficientes $g_{a b}$ del cual se tiene la descomposición 


$$
\mathbf{g}=g_{a b} e^{a} \otimes e^{b},
$$

Es posible obtener el elemento de línea en una base diferente a la determinada por las coordenadas locales y asociarle una métrica plana de manera conveniente, esta es la idea detrás del formalismo de Cartan $[14,15,16]$. La base que vamos a escoger para definir el elemento de línea va a estar constituida por vectores cotangentes $e^{a}$ y donde los coeficientes métricos son los elementos de la matríz $\eta_{a b}$ con la signatura $(-,+,+,+)$.

$$
d s^{2}=e^{a} e^{b} \eta_{a b}
$$

La base $\left\{e^{a}\right\}$ constituye la tétrada o vierbein.

De la relación anterior es difícil observar en que parte está contenida la información de la métrica curva, pero gracias a la relación (2.41) podemos obtener esta información. La sospecha cae sobre la matríz $e_{\mu}^{a}$ que realiza la transformación de la base de coordenadas a la base $e^{a}=e_{\mu}^{a} d x^{\mu}$, que como vemos nos conviene ya que con ella podemos trabajar con una métrica plana al estilo de Minkowski. Esto nos dice que

$$
\begin{aligned}
d s^{2} & =e^{a} e^{b} \eta_{a b} \\
& =e_{\mu}^{a} e_{\nu}^{b} \eta_{a b} d x^{\mu} d x^{\mu} .
\end{aligned}
$$

Es evidente que la información de la curvatura esta contenida de la siguiente forma $g_{\mu \nu}=$ $e_{\mu}^{a} e_{\nu}^{b} \eta_{a b}$; a esta matríz $e_{\mu}^{a}$ que relaciona una transformación lineal de una base de coordenadas a una base $e^{a}$ le llamaremos componentes de la tétrada. Este nuevo elemento $e_{\mu}^{a}$ el cual contiene la información de la curvatura del espacio-tiempo nos proporcionara la relación con la teoría de norma asociada al grupo de simetrías de Poincaré que discutiremos a continuación.

\subsection{El grupo de Poincaré como simetría interna}

En la construcción de la Teoría de Norma requerimos de un grupo interno de simetrías que determinen la norma con la que vamos a obtener una teoría invariante. Para este caso vamos a considerar el grupo de Poincaré para el cual tenemos la siguiente transformación infinitesimal

$$
x^{a} \longrightarrow x^{a}=x^{a}+\varepsilon^{a}+\omega_{b}^{a} x^{b} .
$$

con $a=1,2,3, \ldots$

Cabe mencionar que se utilizaran los indices de las primeras letras del alfabeto $(a, b, c, \ldots)$ para denotar la base de vectores linealmente independientes que identificarán el marco de referencia para la simetría interna. Para la base determinada por una base local, utilizaremos los indices del alfabeto griego $(\mu, \nu, \rho, \ldots)$ para identificar los términos de la métrica $g_{\mu \nu}$.

Bajo está transformación infinitesimal vamos a construir la variación que afectará al campo de matería $\phi(x)^{6}$; primero veamos la forma que toma el campo de matería bajo $x^{\prime}$.

\footnotetext{
${ }^{6} \mathrm{El}$ campo $\phi(x)$, es entendido como un campo auxiliar que sirve para ver el efecto que produce un campo gravitacional
} 
Usando series de Taylor tenemos la forma de la transformación bajo el grupo de Poincaré para el campo $\phi\left(x^{\prime}\right)$

$$
\phi\left(x^{\prime}\right)=\left[1+\left(\omega_{b}^{a} x^{b}+\varepsilon^{a}\right) \partial_{a}\right] \phi(x) .
$$

Haciendo uso del cálculo anterior se realizará una variación inicial $\delta_{0} \phi(x)$ dada por

$$
\delta_{0} \phi(x)=\phi(x)-\phi\left(x^{\prime}\right)=-\left(\varepsilon^{a}+\omega_{b}^{a} x^{b}\right) \partial_{a} \phi(x) .
$$

Reacomodando algunos términos obtenemos lo siguiente:

$$
\begin{aligned}
\delta_{0} \phi(x) & =-\left(\varepsilon^{a} \partial_{a}+\omega_{b}^{a} x^{b} \partial_{a}\right) \phi(x) \\
& =\left[\varepsilon^{a} P_{a}-\frac{1}{2}\left(\omega^{a b}-\omega^{b a}\right) x_{b} \partial_{a}\right] \phi(x) \\
& =\left[\varepsilon^{a} P_{a}-\frac{1}{2} \omega^{a b}\left(x_{b} \partial_{a}-x_{a} \partial_{b}\right)\right] \phi(x) \\
& =\left(\varepsilon^{a} P_{a}+\frac{1}{2} \omega^{a b} M_{a b}\right) \phi(x),
\end{aligned}
$$

con $P_{a}$ y $M_{a b}$ como los generadores de traslaciones y rotaciones respectivamente.

Ahora resulta importante cálcular la variación de la parcial del campo $\phi(x)$ en la base local, bajo la idea de que la transformación infinitesimal depende de la posición ya que en gravitación el espacio-tiempo es curvo. Por lo tanto tenemos que

$$
\begin{aligned}
\delta_{0} \partial_{\mu} \phi(x) & =\partial_{\mu} \delta_{0} \phi(x) \\
& =\partial_{\mu}\left(\varepsilon^{a} P_{a}+\frac{1}{2} \omega^{a b} M_{a b}\right) \phi(x) \\
& =\left(\varepsilon^{a} P_{a}+\frac{1}{2} \omega^{a b} M_{a b}\right) \partial_{\mu} \phi(x)+\left(\partial_{\mu}\left(\varepsilon^{a} P_{a}\right)+\frac{1}{2} \partial_{\mu}\left(\omega^{a b} M_{a b}\right)\right) \phi(x) \\
& =\left(\varepsilon^{a} P_{a}+\frac{1}{2} \omega^{a b} M_{a b}\right) \partial_{\mu} \phi(x)+\frac{1}{2} \partial_{\mu} \omega^{a b} M_{a b} \phi(x)+\partial_{\mu} \varepsilon^{a} P_{a} \phi(x) .
\end{aligned}
$$

Ahora, como podemos observar, la variación de $\partial_{\mu} \phi(x)$ difiere del caso cuando no tenemos dependencia en la posición de la transformación infinitesimal $\varepsilon^{a}+\omega_{b}^{a} x^{b}$. Para construir una teoría invariante de la posición definimos una derivada que nos permita tener una variación sin que se vea el efecto que produce la dependencia en la posición de $\varepsilon^{a}+\omega_{b}^{a} x^{b}$. Para esta construcción vamos a definir la derivada covariante

$$
\nabla_{\mu}:=\partial_{\mu}+A_{\mu}
$$

La aplicación de la variación inicial $\delta_{0}$ a la derivada covariante nos da la siguiente relación 


$$
\begin{aligned}
\delta_{0} \nabla_{\mu} \phi(x) & =\delta_{0}\left(\partial_{\mu}+A_{\mu}\right) \phi(x) \\
& =\delta_{0} \partial_{\mu} \phi(x)+\left(\delta_{0} A_{\mu}\right) \phi(x)+A_{\mu} \delta_{0} \phi(x) .
\end{aligned}
$$

Para facilitar los cálculos posteriores vamos a definir la cantidad

$$
\Theta:=\varepsilon^{a} P_{a}+\frac{1}{2} \omega^{a b} M_{a b}
$$

Para garantizar invarianza debemos obtener una relación similar a la ecuación (2.77); con el fin de lograr esto requerimos que nuestro campo de norma sea igual a $A_{\mu}=\frac{1}{2} \partial_{\mu} \omega^{a b} M_{a b}+$ $\partial_{\mu} \varepsilon^{a} P_{a}$ y además fijamos la variación del campo de norma de la siguiente forma

$$
\left(\delta_{0} A_{\mu}\right) \phi(x):=\left[\Theta, A_{\mu}\right] \phi(x)-A_{\mu} \phi(x) .
$$

Por lo tanto, al sustituir en la ecuación (2.80) los resultados de las ecuaciones (2.77), (2.78), (2.81) y tomando en cuenta el conmutador de $\left[\Theta, A_{\mu}\right] \phi(x)=\left(\Theta A_{\mu}-A_{\mu} \Theta\right) \phi(x)$, tenemos que

$$
\delta_{0} \nabla_{\mu} \phi(x)=\Theta \partial_{\mu} \phi(x)+A_{\mu} \phi(x)+A_{\mu} \Theta \phi(x)+\Theta A_{\mu} \phi(x)-A_{\mu} \Theta \phi(x)-A_{\mu} \phi(x),
$$

y por ende

$$
\begin{aligned}
\delta_{0} \nabla_{\mu} \phi(x) & =\Theta \partial_{\mu} \phi(x)+\Theta A_{\mu} \phi(x) \\
& =\Theta \nabla_{\mu} \phi(x) .
\end{aligned}
$$

Al obtener la forma de la norma $A_{\mu}$, podemos cálcular los invariantes de norma con la ayuda del conmutador $\left[\nabla_{\mu}, \nabla_{\nu}\right]$ que da como resultado lo siguiente

$$
\begin{aligned}
{\left[\nabla_{\mu}, \nabla_{\nu}\right] \phi(x)=} & \left(\partial_{\mu}+A_{\mu}\right)\left(\partial_{\nu}+A_{\nu}\right) \phi(x)-\left(\partial_{\nu}+A_{\nu}\right)\left(\partial_{\mu}+A_{\mu}\right) \phi(x) \\
= & \left(\partial_{\mu} A_{\nu}+A_{\mu} A_{\nu}-\partial_{\nu} A_{\mu}-A_{\nu} A_{\mu}\right) \phi(x) \\
= & \frac{1}{2}\left(\partial_{\mu} \omega_{\nu}^{a b}-\partial_{\nu} \omega_{\mu}^{a b}\right) M_{a b} \phi(x)+\left(\partial_{\mu} e_{\nu}^{a}-\partial_{\nu} e_{\mu}^{a}\right) P_{a} \phi(x) \\
& \frac{1}{2}\left(\omega_{\mu}^{a c} \omega_{\nu}^{d b}-\omega_{\nu}^{a c} \omega_{\mu}^{d b}\right) \eta_{c d} M_{a b} \phi(x)+\left(e_{\mu}^{b} \omega_{\nu}^{c a}-e_{\nu}^{b} \omega_{\mu}^{c a}\right) \eta_{b c} P_{a} \phi(x) \\
= & \frac{1}{2}\left[\partial_{\mu} \omega_{\nu}^{a b}-\partial_{\nu} \omega_{\mu}^{a b}+\left(\omega_{\mu}^{a c} \omega_{\nu}^{d b}-\omega_{\nu}^{a c} \omega_{\mu}^{d b}\right) \eta_{c d}\right] M_{a b} \phi(x) \\
& +\left[\partial_{\mu} e_{\nu}^{a}-\partial_{\nu} e_{\mu}^{a}+\left(e_{\mu}^{b} \omega_{\nu}^{c a}-e_{\nu}^{b} \omega_{\mu}^{c a}\right) \eta_{b c}\right] P_{a} \phi(x) \\
= & \frac{1}{2} F_{\mu \nu}^{a b} M_{a b} \phi(x)+F_{\mu \nu}^{a} P_{a} \phi(x) .
\end{aligned}
$$

Del resultado anterior obtenemos dos objetos contraidos con el generador de rotaciones y traslaciones. Por lo tanto la relación con la teoría gravitacional caerá en identificar a la 
tétrada $e_{\mu}^{a}$ con las cantidades $\partial_{\mu} \varepsilon^{a}$, que tienen que ver con las traslaciones infinitesimales y por ende cómo nos trasladamos en el espacio-tiempo curvo. Por lo tanto, el objeto que está contraido con el generador de traslaciones será relacionado con la torsión, la cual vamos a igualar a cero para garantizar torsión libre como sucede en la formulación de Relatividad General. Como es de esperarse, el término contraido con el generador de rotaciones tendrá relación con la curvatura del espacio-tiempo y el término $\partial_{\mu} M^{a b}=\omega_{\mu}^{a b}$ será el equivalente a los símbolos de Christoffel. A raíz de todo esto tendremos lo siguiente

$$
\begin{gathered}
F_{\mu \nu}^{a b}=\partial_{\mu} \omega_{\nu}^{a b}-\partial_{\nu} \omega_{\mu}^{a b}+\left(\omega_{\mu}^{a c} \omega_{\nu}^{d b}-\omega_{\nu}^{a c} \omega_{\mu}^{d b}\right) \eta_{c d}, \\
F_{\mu \nu}^{a}=\partial_{\mu} e_{\nu}^{a}-\partial_{\nu} e_{\mu}^{a}+\left(e_{\mu}^{c} \omega_{\nu}^{d a}-e_{\nu}^{c} \omega_{\mu}^{d a}\right) \eta_{c d} .
\end{gathered}
$$

Al considerar que existe torsión libre podemos saber explícitamente la forma del término $\omega_{\mu}^{a b}$. Para esto vamos a multiplicar a $F_{\mu \nu}^{d}$ por $e_{b}^{\mu} e_{c}^{\nu} \eta_{d a}$ obteniendo

$$
e_{b}^{\mu} e_{c}^{\nu} \eta_{d a} F_{\mu \nu}^{a}=e_{b}^{\mu} e_{c}^{\nu} \partial_{\mu} e_{\nu a}-e_{b}^{\mu} e_{c}^{\nu} \partial_{\nu} e_{\mu a}+e_{b}^{\mu} e_{c}^{\nu}\left(e_{\mu}^{d} \omega_{\nu d a}-e_{\nu}^{d} \omega_{\mu d a}\right)=0 .
$$

$\mathrm{Al}$ tener esta cantidad con índices libres $a, b$ y $c$, se tomará la permutación en estos índices de la siguiente forma:

$$
\begin{aligned}
e_{b}^{\mu} e_{c}^{\nu} \partial_{\mu} e_{\nu a}-e_{b}^{\mu} e_{c}^{\nu} \partial_{\nu} e_{\mu a}+e_{b}^{\mu} e_{c}^{\nu}\left(e_{\mu}^{d} \omega_{\nu d a}-e_{\nu}^{d} \omega_{\mu d a}\right) \\
+e_{a}^{\mu} e_{b}^{\nu} \partial_{\mu} e_{\nu c}-e_{a}^{\mu} e_{b}^{\nu} \partial_{\nu} e_{\mu c}+e_{a}^{\mu} e_{b}^{\nu}\left(e_{\mu}^{d} \omega_{\nu d c}-e_{\nu}^{d} \omega_{\mu d c}\right) \\
-e_{c}^{\mu} e_{a}^{\nu} \partial_{\mu} e_{\nu b}+e_{c}^{\mu} e_{a}^{\nu} \partial_{\nu} e_{\mu b}-e_{c}^{\mu} e_{a}^{\nu}\left(e_{\mu}^{d} \omega_{\nu d b}-e_{\nu}^{d} \omega_{\mu d b}\right)=0 .
\end{aligned}
$$

Realizando las contracciones pertinentes tenemos que:

$$
\begin{aligned}
& e_{b}^{\mu} e_{c}^{\nu} \partial_{\mu} e_{\nu a}-e_{b}^{\mu} e_{c}^{\nu} \partial_{\nu} e_{\mu a}+e_{c}^{\lambda} \omega_{\lambda b a}-e_{b}^{\lambda} \omega_{\lambda c a} \\
&+e_{a}^{\mu} e_{b}^{\nu} \partial_{\mu} e_{\nu c}-e_{a}^{\mu} e_{b}^{\nu} \partial_{\nu} e_{\mu c}+e_{b}^{\lambda} \omega_{\lambda a c}-e_{a}^{\lambda} \omega_{\lambda b c} \\
&-e_{c}^{\mu} e_{a}^{\nu} \partial_{\mu} e_{\nu b}+e_{c}^{\mu} e_{a}^{\nu} \partial_{\nu} e_{\mu b}-e_{a}^{\lambda} \omega_{\lambda c b}+e_{c}^{\lambda} \omega_{\lambda a b}=0 .
\end{aligned}
$$

Como el objeto $\omega_{\mu a b}$ es antisimétrico en los indices $a b$ es posible tener lo siguiente

$$
\begin{aligned}
2 e_{b}^{\lambda} \omega_{\lambda a c}= & -e_{b}^{\mu} e_{c}^{\nu} \partial_{\mu} e_{\nu a}+e_{b}^{\mu} e_{c}^{\nu} \partial_{\nu} e_{\mu a}-e_{a}^{\mu} e_{b}^{\nu} \partial_{\mu} e_{\nu c}+e_{a}^{\mu} e_{b}^{\nu} \partial_{\nu} e_{\mu c} \\
& +e_{c}^{\mu} e_{a}^{\nu} \partial_{\mu} e_{\nu b}-e_{c}^{\mu} e_{a}^{\nu} \partial_{\nu} e_{\mu b} .
\end{aligned}
$$

Por lo tanto, al multiplicar por $e_{\alpha}^{b}$, obtenemos la forma final de $\omega_{\alpha}^{a c}$; con el cambio de índices $c$ por $b$ y $\alpha$ por $\mu$ tenemos

$$
\omega_{\mu}^{a b}=\frac{1}{2}\left\{e^{b \lambda}\left(\partial_{\lambda} e_{\mu}^{a}-\partial_{\mu} e_{\lambda}^{a}\right)+e^{a \lambda}\left(\partial_{\mu} e_{\lambda}^{b}-\partial_{\lambda} e_{\mu}^{b}\right)+e_{\mu}^{d} e^{a \lambda} e^{b \rho}\left(\partial_{\rho} e_{d \lambda}-\partial_{\lambda} e_{d \rho}\right)\right\} .
$$


Derivado de todo esto podemos obtener las ecuaciones de movimiento en términos de los campos de norma. Como vemos la construcción de la teoría gravitacional puede ser posible a partir de la teoría de norma, donde las ecuaciones de campo están dadas por

$$
F_{\mu}^{a}-\frac{1}{2} F e_{\mu}^{a}=G_{\mu}^{a}
$$

las cuales son determinadas por la construcción del lagrangiano

$$
\mathcal{L}=e F,
$$

donde $e=\operatorname{det}\left(e_{\mu}^{a}\right)$ y $F=F_{\mu \nu}^{a b} e_{a}^{\mu} e_{b}^{\nu}$, el cual es el invariante de norma de curvatura, ya que $F_{\mu \nu}^{a b}$ está relacionado con el tensor de Ricci, $G_{\mu}^{a}$ es el equivalente del tensor de energía momento y $F_{\mu}^{a}=F_{\mu \nu}^{a b} e_{b}^{\nu}$.

\section{Teoría de Norma de De Sitter}

El grupo de Sitter que representa a un hiperboloide $n$ dimensional tiene como motivo describir al espacio-tiempo de la teoría gravitacional de una forma diferente. Esta diferencia radica en considerar que el espacio-tiempo se expande, por lo que en pocas palabras el grupo de de Sitter describe a un espacio-tiempo en expansión.

El grupo de de Sitter se puede formular de una forma similar a la teoría de norma del grupo de Poincaré, con un parámetro a que actua como radio del espacio de Sitter. Una propiedad interesante de este grupo es que cuando el radio a tiende a infinito, éste se reduce al grupo de Poincaré dotando a nuestra teoría la posibilidad de identificar a el radio a como el radio del Universo en expansión.

La estructura del espacio para el grupo de Sitter está constituida por un espacio plano inicial de 5 dimensiones con elemento de línea

$$
\begin{aligned}
d s^{2} & =-\left(d x^{0}\right)^{2}+\left(d x^{1}\right)^{2}+\left(d x^{2}\right)^{2}+\left(d x^{3}\right)^{2}+\left(d x^{5}\right)^{2} \\
& =\eta_{i j} d x^{i} d x^{j}+\left(d x^{5}\right)^{2}
\end{aligned}
$$

Partiendo de esta configuración construimos la hiperesfera $H_{4}$ con radio a contenida en $M_{5}$ y definida por la relación

$$
\eta_{i j} x^{i} x^{j}+\left(x^{5}\right)^{2}=a^{2} .
$$

A partir de la hiperesfera $H_{4}$ podemos cálcular la cantidad $\left(d x^{5}\right)^{2}$ obteniendo

$$
\begin{aligned}
d x^{5} & =d \sqrt{a^{2}-\eta_{i j} x^{i} x^{j}}, \\
& =\frac{1}{2}\left(a^{2}-\eta_{l m} x^{l} x^{m}\right)^{-\frac{1}{2}} d\left(\eta_{i j} x^{i} x^{j}\right), \\
& =\frac{1}{2}\left(a^{2}-\eta_{l m} x^{l} x^{m}\right)^{-\frac{1}{2}} \eta_{i j}\left(d x^{i} x^{j}+x^{i} d x^{j}\right), \\
& =\frac{\eta_{i j} x^{i} d x^{j}}{\sqrt{a^{2}-\eta_{l m} x^{l} x^{m}}} .
\end{aligned}
$$


Utilizando el resultado de (2.89) sobre la línea de mundo de Sitter (2.88), tenemos

$$
\begin{aligned}
d s^{2} & =\eta_{i j} d x^{i} d x^{j}+\frac{\left(\eta_{i j} x^{i} d x^{j}\right)^{2}}{a^{2}-\eta_{l m} x^{l} x^{m}}, \\
& =d x^{i} d x^{j}\left(\eta_{i j}+\frac{x_{i} x_{j}}{a^{2}-\eta_{l m} x^{l} x^{m}}\right) .
\end{aligned}
$$

Con este resultado es posible ver cual es la forma de la métrica en las coordenadas de $H_{4}$

$$
g_{i j}=\eta_{i j}+\frac{x_{i} x_{j}}{a^{2}-\eta_{l m} x^{l} x^{m}} .
$$

La contracción de la métrica con su inversa nos da como resultado $g^{i l} g_{i m}=\delta_{m}^{l}$; a partir de esta relación es posible determinar la forma de la métrica inversa

$$
g^{i j}=\eta^{i j}-\frac{1}{a^{2}} x^{i} x^{j} .
$$

Derivado de la determinación de la métrica inversa es posible observar como se componen los símbolos de Christoffel y el tensor de Ricci. Tenemos que

$$
\begin{aligned}
\Gamma_{j k}^{i} & =\frac{1}{a^{2}}\left[\eta_{j k} x^{i}+\frac{x_{j} x_{k} x^{i}}{a^{2}-\eta_{l m} x^{l} x^{m}}\right], \\
R_{a b} & =\frac{d-1}{a^{2}}\left[\eta_{a b}+\frac{x_{a} x_{b}}{a^{2}-\eta_{l m} x^{m} x^{l}}\right] .
\end{aligned}
$$

Si nos enfocamos en las simétrias es posible encontrar la forma para los campos de norma en la teoría de Sitter. Para esto vamos a considerar las siguientes transformaciones de coordenadas

$$
\begin{aligned}
x^{i} & =a \frac{u^{i}}{u} \sin (u / a), \\
x^{5} & =a \cos (u / a),
\end{aligned}
$$

donde $u=\left(\eta_{a b} u^{a} u^{b}\right)^{\frac{1}{2}}$ y el $\sin (u / a)$, al tener un radio $a$ constante, es posible relacionar el ángulo de abertura con respecto a la quinta coordenada $x^{5}$.

Es relativamente sencillo ver que las transformaciones infinitesimales para $u^{i}$ son

$$
\delta u^{i}=\omega_{j}^{i} u^{j}+\varepsilon^{i} u
$$

En consecuencia las transformaciones infinitesimales para (2.93) con respecto a las coordenadas $u^{i}$ implican lo siguiente 


$$
\begin{aligned}
\delta_{\omega} x^{i} & =\omega_{k}^{j} u^{k} \partial_{j} x^{i} \\
& =\omega_{k}^{j} u^{k} \partial_{j}\left(a \frac{u^{i}}{u} \sin (u / a)\right) \\
& =\omega_{k}^{j} u^{k} a\left(\delta_{j}^{i} \frac{1}{u} \sin (u / a)-\frac{u^{i} u_{j}}{u^{3}} \sin (u / a)+\frac{u^{i} u_{j}}{u^{2} a} \cos (u / a)\right) \\
& =\omega_{j}^{i} a \frac{u^{j}}{u} \sin (u / a) \\
& =\omega_{j}^{i} x^{j},
\end{aligned}
$$

$\mathrm{y}$

$$
\begin{aligned}
\delta_{\omega} x^{5} & =\omega_{k}^{j} u^{k} \partial_{j} x^{5} \\
& =\omega_{k}^{j} u^{k} \partial_{j}(a \cos (u / a)) \\
& =-\omega_{k}^{j} u^{k} u_{j} \frac{\sin (u / a)}{u} \\
& =-\omega^{j k} u_{j} u_{k} \frac{\sin (u / a)}{u} \\
& =0 .
\end{aligned}
$$

Para el último resultado tenemos un cero ya que el objeto antisimétrico $\omega^{i j}=-\omega^{j i}$ está contraido con un término simétrico $u_{j} u_{k}$. Sigamos cálculando para el párametro de desplazamiento tanto para $x^{i}$ como para $x^{5}$. Para ello consideramos las variaciones

$$
\begin{aligned}
\delta_{\varepsilon} x^{i} & =u \varepsilon^{j} \partial_{j} x^{i} \\
& =u \varepsilon^{j} \partial_{j}\left(a \frac{u^{i}}{u} \sin (u / a)\right) \\
& =u \varepsilon^{j} a\left(\delta_{j}^{i} \frac{1}{u} \sin (u / a)-\frac{u^{i} u_{j}}{u^{3}} \sin (u / a)+\frac{u^{i} u_{j}}{u^{2} a} \cos (u / a)\right) \\
& =\frac{u}{u} u \varepsilon^{i} a \frac{1}{u} \sin (u / a)-\frac{u^{i} \varepsilon_{j} u^{j}}{u} a \frac{1}{u} \sin (u / a)+\frac{\varepsilon^{j} u_{j}}{u a} u^{i} a \cos (u / a) \\
& =\frac{\varepsilon^{i} u_{j}}{u} x^{j}-\frac{u^{i} \varepsilon_{j}}{u} x^{j}+\frac{\varepsilon^{j} u_{j}}{u a} u^{i} x^{5} \\
& =\frac{1}{u}\left(\varepsilon^{i} u_{j}-\varepsilon_{j} u^{i}\right) x^{j}+\frac{\varepsilon^{j} u_{j}}{u a} u^{i} x^{5},
\end{aligned}
$$




$$
\begin{aligned}
\delta_{\varepsilon} x^{5} & =u \varepsilon^{i} \partial_{i} x^{5} \\
& =u \varepsilon^{i} \partial_{i}(a \cos (u / a)) \\
& =-\varepsilon^{i} u a \sin (u / a) \frac{u_{i}}{u a} \\
& =-\frac{\varepsilon^{j} u_{j}}{u a} u_{i} x^{i} .
\end{aligned}
$$

Las transformaciones infinitesimales de de Sitter nos proporcionan los siguientes parámetros de transformación $\omega^{i j}=1 / u\left(\varepsilon^{i} u^{j}-\varepsilon^{j} u^{i}\right), \omega^{i 5}=\frac{u^{i}(u \cdot \varepsilon)}{u a}$. Por lo tanto la forma de los generadores de traslaciones y rotaciones en las coordenadas $u^{i}$, se corresponden a través de los parámetros de traslación. Para esto definimos el vector $q_{j}{ }^{i}$ como la diferencial de las transformaciones infinitesimales $x^{i}$, con respecto a los parmetros de transformaciones $\zeta^{i}$,

$$
q_{j}{ }^{i}=\left(\frac{\partial x^{i}}{\partial \zeta^{j}}\right)_{\zeta^{j}=0},
$$

Por lo tanto los generadores de las transformaciones son

$$
P_{i}=q_{i}{ }^{j}\left(\frac{\partial}{\partial x^{j}}\right)
$$

con $x^{i}$ como las coordenadas del espacio-tiempo.

En primera instancia es posible ver que el generador de rotaciones para las coordenadas $u_{i}$ es:

$$
M_{i j}(u)=u_{i} \partial_{j}-u_{j} \partial_{i}
$$

ya que la ecuación de transformación (2.95) hace evidente que la transformación del parámetro $\omega^{i j}$ es invariante ante transformaciones de coordenas entre $x^{i}$ y $u^{i}$, pero ¿Qué sucede para el generador de traslaciones?, y ¿Qué generador se derivada del objeto $\omega^{i 5}$ ? Para contestar estas preguntas, primero vamos a cálcular la parcial de (2.97) con respecto del parámetro $u \varepsilon_{i}$

$$
\begin{aligned}
q_{j}^{i} & =\left(\frac{\partial \delta_{\varepsilon} x^{i}}{\partial u \varepsilon^{j}}\right)_{u \varepsilon^{j}=0} \\
& =\frac{1}{u^{2}}\left(\delta_{j}^{i} u_{k}-\eta_{k j} u^{i}\right) x^{k}+\frac{u_{j} u^{i}}{u^{2} a} x^{5} .
\end{aligned}
$$

Entonces el generador $P_{i}$ queda como

$$
\begin{aligned}
P_{i} & =q_{i}{ }^{j} \frac{\partial}{\partial x^{j}} \\
& =\frac{1}{u}\left\{\frac{1}{u}\left(\delta_{i}{ }^{j} u_{k}-\eta_{k i} u^{j}\right) x^{k}+\frac{u_{i} u^{j}}{u a} x^{5}\right\} \frac{\partial}{\partial x^{j}} .
\end{aligned}
$$


De la relación anterior no se ve claro que tipo de generador tenemos, pero si tomamos en cuenta que

$$
\begin{aligned}
\frac{\partial}{\partial u^{i}} & =\frac{\partial x^{j}}{\partial u^{i}} \frac{\partial}{\partial x^{j}} \\
& =\frac{\partial}{\partial u^{i}}\left(a \frac{u^{j}}{u} \sin (u / a)\right) \frac{\partial}{\partial x^{j}} \\
& =\left(\delta_{i}{ }^{j} \frac{a}{u} \sin (u / a)-\frac{a u^{j} u_{i}}{u^{3}} \sin (u / a)+\frac{u^{j} u_{i}}{u^{2}} \cos (u / a)\right) \frac{\partial}{\partial x^{j}} \\
& =\frac{1}{u}\left[\frac{1}{u}\left(\delta_{i}{ }^{j} u_{k} x^{k}-u_{i} x^{j}\right)+\frac{u_{i} u^{j}}{u a} x^{5}\right] \frac{\partial}{\partial x^{j}},
\end{aligned}
$$

entonces de la ecuación (2.104) vemos que (2.103) queda simplemente como

$$
P_{i}=\frac{\partial}{\partial u^{i}}
$$

Por lo tanto $P_{i}$ es nuestro generador de traslaciones para las coordenadas $u^{i}$, pero aun queda por saber que interpretación tiene $\omega^{i 5}$. Calculamos entonces la cantidad

$$
\begin{aligned}
q_{i 5}^{5} & =\left(\frac{\partial \delta_{\varepsilon} x^{5}}{\partial \omega^{i 5}}\right)_{\omega^{i 5}=0} \\
& =\left(-\frac{\partial}{\partial \omega^{i 5}} \frac{\varepsilon^{k} u_{k}}{u a} \eta_{l m} u^{l} x^{m}\right)_{\omega^{i 5}=0} \\
& =\left(-\frac{\partial}{\partial \omega^{i 5}} \eta_{l m} \omega^{l 5} x^{m}\right)_{\omega^{i 5}=0} \\
& =-\eta_{i m} x^{m}
\end{aligned}
$$

por lo que el generador $P_{i 5}$ será

$$
\begin{aligned}
M_{i 5} & =q_{i 5}{ }^{5} \frac{\partial}{\partial x^{5}} \\
& =-\eta_{i m} x^{m} \frac{\partial}{\partial x^{5}} .
\end{aligned}
$$

De aquí se hace díficil ver que generador se deriva de la coordenada $x^{5}$, por lo que vamos a ver que relación guarda la parcial de $x^{5}$ y $u^{i}$. Utilizando regla de la cadena tenemos

$$
\begin{aligned}
\frac{\partial}{\partial u^{i}} & =\frac{\partial x^{5}}{\partial u^{i}} \frac{\partial}{\partial x^{5}} \\
& =-\frac{u_{i}}{u} \sin (u / a) \frac{\partial}{\partial x^{5}} \\
& =-\frac{1}{a} \eta_{i j} x^{j} \frac{\partial}{\partial x^{5}}
\end{aligned}
$$


De las relaciones (2.107) vemos que

$$
\begin{aligned}
M_{i 5} & =a \frac{\partial}{\partial u^{i}} \\
& =a P_{i} .
\end{aligned}
$$

Entonces el generador de traslaciones para el grupo de de Sitter es simplemente

$$
P_{i}=\frac{1}{a} M_{i 5}
$$

Contando con los generadores para el espacio de de Sitter, es posible saber como es la forma de los tensores $F_{\mu \nu}^{i j}$ y $F_{\mu \nu}^{i}$ derivados de la teoría de norma para el grupo de Poincaré y tomando en consideración que el campo de norma $e_{\mu}^{i}=a \omega_{\mu}^{i 5}$, tenemos lo siguiente:

$$
\begin{aligned}
F_{\mu \nu}^{i j} & =\partial_{\mu} \omega_{\nu}^{i j}-\partial_{\nu} \omega_{\mu}^{i j}+\left(\omega_{\mu}^{i k} \omega_{\nu}^{l j}-\omega_{\nu}^{i k} \omega_{\mu}^{l j}\right) \eta_{k l}+\left(\omega_{\mu}^{i 5} \omega_{\nu}^{5 j}-\omega_{\nu}^{i 5} \omega_{\mu}^{5 j}\right) \\
& =\partial_{\mu} \omega_{\nu}^{i j}-\partial_{\nu} \omega_{\mu}^{i j}+\left(\omega_{\mu}^{i k} \omega_{\nu}^{l j}-\omega_{\nu}^{i k} \omega_{\mu}^{l j}\right) \eta_{k l}-\frac{1}{a^{2}}\left(e_{\mu}^{i} e_{\nu}^{j}-e_{\nu}^{i} e_{\mu}^{j}\right)
\end{aligned}
$$

y además

$$
\begin{aligned}
F_{\mu \nu}^{i 5} & =\frac{1}{a} T_{\mu \nu}^{i} \\
& =\frac{1}{a}\left[\partial_{\mu} e_{\nu}^{i}-\partial_{\nu} e_{\mu}^{i}+\left(e_{\mu}^{j} \omega_{\nu}^{k i}-e_{\nu}^{j} \omega_{\mu}^{k i}\right) \eta_{j k}\right]
\end{aligned}
$$

De esta forma obtenemos la teoría de norma de de Sitter, que está vinculada con la constante cosmológica, la cual será utilizada más adelante para la deformación de la teoría de norma. 


\section{Capítulo 3}

\section{Deformación de la Teoría de Norma para Gravedad}

Anteriormente discutimos la construcción de la teoría de norma para gravedad lo cual introduce nuevos elementos llamados campos de norma. Los cuales serán afectados por la aplicación de Seiberg-Witten para obtener campos de norma no-conmutativos que darán origen a la teoría gravitacional deformada. Para esto vamos a introducir algunos conceptos necesarios para comprender el porqué de este tipo de deformación.

\subsection{Producto de Moyal-Weyl}

Para la construcción de un espacio no-conmutativo se introducen las siguientes relaciones de conmutación

$$
\left[x^{\mu}, x^{\nu}\right]=i \Theta^{\mu \nu}
$$

Los elementos de $\Theta^{\mu \nu}$ son antisimétricos y en general son funciones de las coordenadas. A esta relación de conmutación la nombraremos forma canónica. En este espacio no-conmutativo se pueden obtener teorías de campo remplazando el producto normal con el producto- $\star$ de la siguiente forma

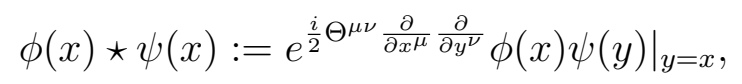

donde consideramos a partir de ahora que los elementos $\Theta^{\mu \nu}$ son constantes. Este producto-

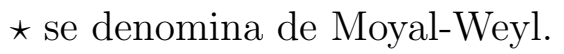

En el caso de una relación de conmutación tipo álgebra de Lie $\left[x^{\mu}, x^{\nu}\right]=i \Theta_{\lambda}^{\mu \nu} x^{\lambda}$, el producto estrella adquiere la siguiente forma

$$
\phi(x) \star \psi(x)=\left.e^{\frac{i}{2} x^{\mu} g_{\mu}\left(i \frac{\partial}{\partial y^{\mu}}, \frac{\partial}{\partial z^{\nu}}\right)} \phi(x) \psi(y)\right|_{y=x=z},
$$

donde $g_{\mu}$ es una función que depende de sus argumentos; notese la dependencia en $x^{\mu}$ en el argumento de la exponencial.

El producto estrella tiene una propiedad muy importante la cual es 


$$
\int d^{n} x \phi(x) \star \psi(x)=\int d^{n} x \psi(x) \star \phi(x)=\int d^{n} x \phi(x) \psi(x) .
$$

Para el caso de 3 ó mas campos se tiene que

$$
\begin{aligned}
\int d^{n} x\{\phi(x) \star \psi(x)\} \star \rho(x) & \neq \int d^{n} x\{\phi(x) \star \psi(x)\} \rho(x) \\
& \neq \int d^{n} x \phi(x)\{\psi(x) \star \rho(x)\} \\
& =\int d^{n} x \phi(x) \star\{\psi(x) \star \rho(x)\} .
\end{aligned}
$$

La contrucción de los campos de norma no-conmutativos deriva de considerar la variación del campo de matería no-conmutativo $\hat{\psi}(x)$. La transformación infinitesimal bajo el parámetro de norma no-conmutativo esta dada por

$$
\delta_{\hat{\Lambda}} \hat{\psi}=i \hat{\Lambda} \star \hat{\psi}
$$

la cual tiene la misma forma funcional que el caso conmutativo.

El parámetro de norma no-conmutativo es una función con valores en el álgebra universal envolvente $\mathcal{U}$. Ya que el grupo de simétrias que deriva de nuestros campos de norma es noabeliano, la determinación de los campos de norma a partir de la variación del campo de matería no-conmutativo da como resultado una infinidad de campos de norma, lo cual no es útil por el simple hecho de obtener más grados de libertad de los que teníamos. Para resolver este problema inicial vamos a utilizar la aplicación de Seiberg-Witten [6, 11] para reducir el número de grados de libertad. De igual modo vamos a utilizar el álgebra universal envolvente $\mathcal{U}$, la cual está construida por productos de los elementos del álgebra [17].

De este modo, un elemento general del álgebra universal envolvente $\mathcal{U}$ en composición con los generadores del grupo de simetrías $T_{a}$, se escribe de la siguiente manera

$$
\alpha(x)+\alpha^{a}(x) T_{a}+\alpha^{a b}(x) T_{a} T_{b}+\ldots
$$

\subsection{Aplicación de Seiberg-Witten}

La aplicación de Seiberg-Witten nos ayuda para la definición y conformación de los campos de norma no-conmutativos a partir de los campos de norma conmutativos de modo tal que posean los mismos grados de libertad; esto resuelve el problema anteriormente expresado ya que no es conveniente trabajar con más grados de libertad que con los que empezamos.

Por lo tanto empezamos considerando los campos no-conmutativos $\hat{\psi}, \hat{A}_{\mu}$ y el parámetro de norma como una función de sus contrapartes conmutativas, del parámetro de norma y con un parámetro de deformación $\theta$ constante

$$
\hat{\psi}(\psi, A, \theta) \quad, \hat{A}_{\mu}(A, \theta) \quad, \hat{\Lambda}(\Lambda, A, \theta) .
$$


En analogía con las transformaciones infinitesimales de los campos y el parámetro de norma conmutativos, se toman las siguientes transformaciones infinitesimales del parámetro de norma para el campo de matería y de norma no-conmutativa

$$
\begin{gathered}
\delta_{\hat{\Lambda}} \hat{\psi}=i \hat{\Lambda} \star \hat{\psi}, \\
\delta_{\hat{\Lambda}} \hat{A}_{\mu}=\partial_{\mu} \hat{\Lambda}+i\left[\hat{\Lambda}, \hat{A}_{\mu}\right]_{\star} .
\end{gathered}
$$

La definición de la aplicación de Seiberg-Witten que relaciona términos de campo noconmutativos con los términos de campo ordinarios, surge de la idea de un cierto límite a la teoría de cuerdas [18] conocido como equivalencia de norma

$$
\delta_{\hat{\Lambda}} \hat{A}_{\mu}(A ; \theta)=\hat{A}_{\mu}\left(A+\delta_{\alpha} A ; \theta\right)-\hat{A}_{\mu}(A ; \theta)=\delta_{\alpha} \hat{A}_{\mu}(A ; \theta) .
$$

Para la resolución de la ecuación (3.8) es necesario tener en consideración la consistencia de norma no-conmutativa o también llamada condición de cociclo

$$
i \delta_{\alpha} \hat{\Lambda}_{\beta}-i \delta_{\beta} \hat{\Lambda}_{\alpha}-\left[\hat{\Lambda}_{\alpha}, \hat{\Lambda}_{\beta}\right]_{\star}=i \hat{\Lambda}_{-i[\alpha, \beta]} .
$$

Gracias a esta relación es posible encontrar el parámatro de norma no-conmutativo $\hat{\Lambda}_{\alpha}$, y por lo tanto también se determinará el campo de norma $\hat{A}_{\mu}$. Bajo estas condiciones se pueden tener a $\hat{\Lambda}_{\alpha}$ y $\hat{A}_{\mu}$ como una expansión en series de potencia en $\theta$ de la siguiente forma

$$
\begin{aligned}
& \hat{\Lambda}_{\alpha}=\alpha+\Lambda_{\alpha}^{1}+\Lambda_{\alpha}^{2}+\ldots, \\
& \hat{A}_{\mu}=A_{\mu}+A_{\mu}^{1}+A_{\mu}^{2}+\ldots,
\end{aligned}
$$

Esto nos permite re-definir a las variaciones de los campos no-conmutativos para cualquier orden en $n$ como:

$$
\Delta \Lambda^{n}:=i \delta_{\alpha} \hat{\Lambda}_{\beta}^{n}-i \delta_{\beta} \hat{\Lambda}_{\alpha}^{n}-\left[\alpha, \hat{\Lambda}_{\beta}^{n}\right]-\left[\hat{\Lambda}_{\alpha}^{n}, \beta\right]-i \hat{\Lambda}_{-i[\alpha, \beta]}^{n}=-\sum_{p+q+r=n}\left[\Lambda_{\alpha}^{p}, \Lambda_{\beta}^{q}\right]_{\star^{r}}
$$

$\operatorname{con} p, q \neq n \mathrm{y}$

$$
\Delta_{\alpha} A_{\mu}^{n}=\delta_{\alpha} A_{\mu}^{n}-i\left[\alpha, A_{\mu}^{n}\right]=\partial_{\mu} \Delta_{\alpha}^{n}+i \sum_{p+q+r=n}\left[\Lambda_{\alpha}^{p}, A_{\mu}^{q}\right]_{\star^{r}}
$$

con $p, q \neq n$, tomando en cuenta que el producto estrella a $r$-ésimo término es

$$
f(x) \star^{r} g(x)=\frac{1}{r !}\left(\frac{i}{2}\right)^{r} \theta^{\mu_{1} \nu_{1}} \cdots \theta^{\mu_{r} \nu_{r}} \partial_{\mu_{1}} \cdots \partial_{\mu_{r}} f(x) \partial_{\nu_{1}} \cdots \partial_{\nu_{r}} g(x),
$$

lo cual nos permite recuperar la parte conmutativa en el límite cuando $\theta \longrightarrow 0$. Para conocer la solución de los términos no-conmutativios orden por orden tenemos que hacer uso de las relaciones (3.10), (3.11). En la solución general es posible tomar soluciones homogéneas de las relaciones (3.10) y (3.11)con coeficientes arbitrarios como se menciona en $[6,19,20]$. 
Estos términos se conocen como ambiguedades y fueron una fuente de confución al inicio de los trabajos en teoría de campos no-conmutativos.

Como una consecuencia de la forma en como están constituidas las condiciones para (3.10) y (3.11), es posible determinar de forma recursiva del orden más bajo a un orden superior los componentes para $\Lambda^{n}$ y $A_{\mu}^{n}$ como se expone en [6]

$$
\begin{gathered}
\Lambda_{\alpha}^{n+1}=-\frac{\theta^{\mu \nu}}{4(n+1)} \sum_{p+q+r=n}\left\{A_{\mu}^{p}, \partial_{\nu} \Lambda_{\alpha}^{q}\right\}_{\star^{r}}, \\
A_{\mu}^{n+1}=-\frac{\theta^{\sigma \rho}}{4(n+1)} \sum_{p+q+r=n}\left\{A_{\sigma}^{p}, \partial_{\rho} A_{\mu}^{q}+F_{\rho \mu}^{q}\right\}_{\star^{r}} .
\end{gathered}
$$

Una vez que se tienen determinados los campos de matería y de norma a orden $n$, es posible determinar la curvatura $F_{\mu \nu}^{n}$ asociada, la cual está determinada por medio de la expresión

$$
\hat{F}_{\mu \nu}=\partial_{\mu} \hat{A}_{\nu}-\partial_{\nu} \hat{A}_{\mu}-i\left[\hat{A}_{\mu}, \hat{A}_{\nu}\right]_{\star}=F_{\mu \nu}+F_{\mu \nu}^{(1)}+F_{\mu \nu}^{(2)}+\ldots
$$

Explicitamente sus componentes son

$$
\begin{aligned}
F_{\mu \nu}^{n+1} & =-\frac{\theta^{\sigma \rho}}{4(n+1)} \sum_{p+q+r=n}\left(\left\{A_{\sigma}^{p}, \partial_{\rho} F_{\mu \nu}^{q}+\left(D_{\rho} F_{\mu \nu}\right)^{q}\right\}_{\star^{r}}-2\left\{F_{\mu \sigma}^{p}, F_{\nu \rho}^{q}\right\}_{\star^{r}}\right), \\
\left(D_{\mu} F_{\sigma \rho}\right)^{n} & =\partial_{\mu} F_{\sigma \rho}^{n}-i \sum_{p+q+r=n}\left[A_{\mu}^{p}, F_{\sigma \rho}^{q}\right]_{\star^{r}},
\end{aligned}
$$

a orden $n$. Por lo tanto, si se conocen los campos conmutativos iniciales, las relaciones anteriores permiten la construcción de la teoría de campos no-conmutativa a orden $n$ en el parámetro no-conmutativo

\subsection{Grupo de simetrías no-conmutativas}

Con la determinación de los campos de norma deformados por medio de la aplicación de Seiberg-Witten, en primera instancia se puede aplicar el formalismo anterior a la Relatividad General como una teoría tomando en cuenta el grupo de simetrías que corresponde a la construcción de la teoría gravitacional.

Para este caso se toma el campo de norma siguiente $A_{\mu}=\frac{1}{2} \omega_{\mu}^{a b} M_{a b}+e_{\mu}^{a} P_{a}$, que resulta de la teoría de norma gravitacional como se discutió anteriormente. Como es evidente observar, se derivan dos expresiones en la deformación para los campos conmutativos $e_{\mu}^{a}$ y $\omega_{\mu}^{a b}$ que vamos a identificar como $\hat{e}_{\mu}^{a}$ y $\hat{\omega}_{\mu}^{a b}$. Al desarrollar en serie de potencias en el parámetro no-conmutativo $\theta$, para ambos términos tenemos que

$$
\begin{aligned}
\hat{\omega}_{\mu}^{a b}(x, \theta) & =\omega_{\mu}^{a b}(x)-i \omega_{\mu}^{a b 1}(x, \theta)+\omega_{\mu}^{a b 2}(x, \theta)+\ldots+\omega_{\mu}^{a b n}, \\
& =\omega_{\mu}^{a b}(x)-i \theta^{\nu \rho} \omega_{\mu \nu \rho}^{a b}(x)+\theta^{\nu \rho} \theta^{\lambda \tau} \omega_{\mu \nu \rho \lambda \tau}^{a b}(x)+\ldots \mathcal{O}\left(\theta^{n}\right),
\end{aligned}
$$




$$
\begin{aligned}
\hat{e}_{\mu}^{a}(x, \theta) & =e_{\mu}^{a}(x)-i e_{\mu}^{a 1}(x)+e_{\mu}^{a 2}(x)+\ldots+e_{\mu}^{a n}(x) \\
& =e_{\mu}^{a}(x)-i \theta^{\nu \rho} e_{\mu \nu \rho}^{a}(x)+\theta^{\nu \rho} \theta^{\lambda \tau} e_{\mu \nu \rho \lambda \tau}^{a}(x)+\ldots \mathcal{O}\left(\theta^{n}\right) .
\end{aligned}
$$

Por lo tanto los términos no-conmutativos a cualquier orden se identificarán como

$$
A_{\mu}^{N}=\frac{1}{2} \omega_{\mu}^{a b N} M_{a b}+e_{\mu}^{a N} P_{a}
$$

con el índice $N$ etiquetando el enésimo término de deformación. Al sustituir la forma del campo de norma $A_{\mu}^{N}$ en la relación (3.13) tenemos que ${ }^{1}$

$$
\begin{aligned}
A_{\mu}^{N+1}= & -\frac{\theta^{\sigma \rho}}{4(n+1)} \sum_{P+Q+R=N}\left\{\frac{1}{2} \omega_{\sigma}^{a b P} M_{a b}+e_{\sigma}^{a P} P_{a}, \partial_{\rho}\left(\frac{1}{2} \omega_{\mu}^{c d} M_{c d}+e_{\mu}^{c Q} P_{c}\right)\right. \\
& \left.+\frac{1}{2} F_{\rho \mu}^{c d} M_{c d}\right\}_{\star^{R}} \\
= & -\frac{\theta^{\sigma \rho}}{4(n+1)} \sum_{P+Q+R=N}\left[\frac{1}{4}\left(\omega_{\sigma}^{a b P} \star^{R} \partial_{\rho} \omega_{\mu}^{c d}{ }^{Q} M_{a b} M_{c d}+\partial_{\rho} \omega_{\mu}^{c d} \star^{R} \omega_{\sigma}^{a b P} M_{c d} M_{a b}\right)\right. \\
& +\frac{1}{4}\left(\omega_{\sigma}^{a b P} \star^{R} F_{\rho \mu}^{c d} M_{a b} M_{c d}+F_{\rho \mu}^{c d} \star^{R} \omega_{\sigma}^{a b P} M_{c d} M_{a b}\right) \\
& \left.+\frac{1}{2} \partial_{\rho} e_{\mu}^{c Q} \star^{R} \omega_{\sigma}^{a b P} P_{c} M_{a b}+\frac{1}{2} e_{\sigma}^{a P} \star^{R} \partial_{\rho} \omega_{\mu}^{c d}{ }^{c} P_{a} M_{c d}+\frac{1}{2} e_{\sigma}^{a P} \star^{R} F_{\rho \mu}^{c d} P_{a} M_{c d}\right] \\
= & -\frac{\theta^{\sigma \rho}}{4(n+1)} \sum_{P+Q+R=N}\left[\frac{1}{2}\left\{\omega_{\sigma}^{P}, \partial_{\rho} \omega_{\mu}^{Q}+F_{\rho \mu}^{Q}\right\}_{\star}^{a b} M_{a b}\right. \\
& \left.-\left(\omega_{\sigma}^{a b P} \star^{R} \partial_{\rho} e_{\mu}^{d Q}+\partial_{\rho} \omega_{\mu}^{a c Q} \star^{R} e_{\sigma}^{d P}+F_{\rho \mu}^{a c Q} \star^{R} e_{\sigma}^{d P}\right) \eta_{c d} P_{a}\right] .
\end{aligned}
$$

Comparando las ecuaciones (3.16) y la relación (3.17), es posible observar orden por orden la forma de los campos deformados los cuales son:

$$
\begin{gathered}
\omega_{\mu}^{a b N+1}=-\frac{\theta^{\sigma \rho}}{4(n+1)} \sum_{P+Q+R=N}\left\{\omega_{\sigma}^{P}, \partial_{\rho} \omega_{\mu}^{Q}+F_{\rho \mu}^{Q}\right\}_{\star R}^{a b}, \\
e_{\mu}^{a N+1}=\frac{\theta^{\sigma \rho}}{4(n+1)} \sum_{P+Q+R=N}\left[\omega_{\sigma}^{a c P} \star^{R} \partial_{\rho} e_{\mu}^{d Q}+\left(\partial_{\rho} \omega_{\mu}^{a c Q}+F_{\rho \mu}^{a c} Q\right) \star^{R} e_{\sigma}^{d P}\right] \eta_{c d}
\end{gathered}
$$

donde

$$
\begin{aligned}
\{\tau, \lambda\}^{a b} & :=\left(\tau^{a c} \lambda^{d b}+\lambda^{a c} \tau^{d b}\right) \eta_{c d}, \\
{[\tau, \lambda]^{a b} } & :=\left(\tau^{a c} \lambda^{d b}-\lambda^{a c} \tau^{d b}\right) \eta_{c d} .
\end{aligned}
$$

${ }^{1} \mathrm{Al}$ tener torsión libre el objeto $F_{\mu \nu}^{a N}$ es igual a cero 
Ya que se conocen los campos de norma deformados en general, es posible obtener de (3.18) y (3.19) los términos de los campos de norma deformados a primer y segundo orden en $\theta$. Tenemos así

$$
\begin{aligned}
\omega_{\mu}^{a b 1}= & -\frac{\theta^{\sigma \rho}}{4}\left\{\omega_{\sigma}, \partial_{\rho} \omega_{\mu}+F_{\rho \mu}\right\}^{a b}, \\
\omega_{\mu}^{a b 2}= & \frac{\theta^{\sigma \rho} \theta^{\lambda \tau}}{32}\left[\left\{\left\{\omega_{\lambda}, \partial_{\tau} \omega_{\sigma}+F_{\tau \sigma}\right\}, \partial_{\rho} \omega_{\mu}+F_{\rho \mu}\right\}^{a b}\right. \\
& \left.+\left\{\omega_{\sigma}, \partial_{\rho}\left\{\omega_{\lambda}, \partial_{\tau} \omega_{\mu}+F_{\tau \mu}\right\}+\left\{\omega_{\lambda}, 2 \partial_{\tau} F_{\rho \mu}\right\}-2\left\{F_{\rho \lambda}, F_{\mu \tau}\right\}\right\}^{a b}\right] \\
& -i \frac{\theta^{\sigma \rho} \theta^{\lambda \tau}}{32}\left[\left\{\omega_{\sigma},\left\{\omega_{\lambda},\left[\omega_{\tau}, F_{\rho \mu}\right]\right\}\right\}^{a b}\right. \\
& \left.+2\left\{\partial_{\lambda} \omega_{\sigma}, \partial_{\tau}\left(\partial_{\rho} \omega_{\mu}+F_{\rho \mu}\right)\right\}^{a b}\right],
\end{aligned}
$$

y además

$$
\begin{aligned}
e_{\mu}^{a 1}= & \frac{\theta^{\sigma \rho}}{4}\left[\omega_{\sigma}^{a c} \partial_{\rho} e_{\mu}^{d}+\left(\partial_{\rho} \omega_{\mu}^{a c}+F_{\rho \mu}^{a c}\right) e_{\sigma}^{d}\right] \eta_{c d}, \\
e_{\mu}^{a 2}= & \frac{\theta^{\sigma \rho} \theta^{\lambda \tau}}{32}\left[\left(\partial_{\rho} \omega_{\mu}^{a c}+F_{\rho \mu}^{a c}\right)\left(\omega_{\lambda}^{d e} \partial_{\tau} e_{\sigma}^{f}+\left(\partial_{\tau} \omega_{\sigma}^{d e}+F_{\tau \sigma}^{d e}\right) e_{\lambda}^{f}\right) \eta_{e f}\right. \\
& +\omega_{\sigma}^{a c} \partial_{\rho}\left(\omega_{\lambda}^{d e} \partial_{\tau} e_{\mu}^{f}+\left(\partial_{\tau} \omega_{\mu}^{d e}+F_{\tau \mu}^{d e}\right) e_{\lambda}^{f}\right) \eta_{e f} \\
& -2\left(\left\{\omega_{\lambda}, \partial_{\tau} F_{\rho \mu}\right\}^{a c}-\left\{F_{\rho \lambda}, F_{\mu \tau}\right\}^{a c}\right) e_{\sigma}^{d} \\
& -\partial_{\rho}\left\{\omega_{\lambda}, \partial_{\tau} \omega_{\mu}+F_{\tau \mu}\right\}^{a c} e_{\sigma}^{d} \\
& \left.-\left\{\omega_{\lambda}, \partial_{\tau} \omega_{\sigma}+F_{\tau \sigma}\right\}^{a c} \partial_{\rho} e_{\mu}^{d}\right] \eta_{c d} \\
& +i \frac{\theta^{\sigma \rho} \theta^{\lambda \tau}}{32}\left[\left\{\omega_{\lambda},\left[\omega_{\tau}, F_{\rho \mu}\right]\right\}^{a c} e_{\sigma}^{d}+2 \partial_{\lambda} \omega_{\sigma}^{a c} \partial_{\tau} \partial_{\rho} e_{\mu}^{d}\right. \\
& \left.+2 \partial_{\lambda}\left(\partial_{\rho} \omega_{\mu}^{a c}+F_{\rho \mu}^{a c}\right) \partial_{\tau} e_{\sigma}^{d}\right] \eta_{c d} .
\end{aligned}
$$

Se observa que la tétrada deformada es una cantidad compleja por lo que la métrica deformada se define como una cantidad real dad por

$$
\hat{g}_{\mu \nu}:=\frac{1}{2} \eta_{a b}\left(\hat{e}_{\mu}^{a} \star \hat{e}_{\nu}^{b \dagger}+\hat{e}_{\mu}^{a \dagger} \star \hat{e}_{\nu}^{b}\right)
$$

Esta definición nos lleva a considerar la parte conjugada $\hat{e}_{\mu}^{a \dagger}$ para la tétrada deformada $[21,22]$. Como hemos visto, la deformación de los campos de norma es posible construir la teoría gravitacional a partir de la tétrada deformada $\hat{e}_{\mu}^{a}$, lo cual trataremos en los siguientes capítulos para dos espacio-tiempo específicos. 


\section{Capítulo 4}

\section{Teoría Gravitacional No-Conmutativa}

Una de las formas para vislumbrar los acontecimientos de la naturaleza es mediante la aplicación de teorías que proporcionen un mejor entendimiento del entorno. Para este capítulo vamos a aplicar la teoría de deformación de campos basada en la aplicación de Seiberg-Witten para la teoría de gravitación. Para ilustrar este procedimiento se van a considerar para la deformación a dos métricas que representan hoyos negros con parámetro de rotación; las cuales son la solución BTZ [4] y Kerr-Newman [5] con rotación lenta.

Para cálcular los campos de norma deformados vamos a tomar en consideración la siguiente forma de la matríz de deformación $\theta^{\mu \nu}$.

$$
\theta^{\mu \nu}=\left[\begin{array}{ccc}
0 & 0 & 0 \\
0 & 0 & \theta \\
0 & -\theta & 0
\end{array}\right]
$$

Como es posible observar, estamos imponiendo que la no conmutación de las coordenadas sea entre $r$ y $\theta$. Este caso solo aplica para la métrica BTZ, para el caso de Kerr-Newman con rotación lenta tendríamos una matríz similar pero extendida para un espacio 4-dimensional.

Basándonos en las relaciones (3.22) y (3.23), calcularemos la deformación de la tétrada que posteriormente será utilizada para observar las diferencias con respecto a la teoría gravitacional sin deformar.

\subsection{BTZ No-Conmutativo}

Realizar un análisis para un espacio $(2+1)$-dimensional supone un mejor manejo en los cálculos que podría facilitar la manipulación y análisis de la teoría gravitacional en el caso más general. Bajo esta idea es como escogemos a la métrica BTZ [4] (representación de un hoyo negro $(2+1)$-dimensional con parámetro de rotación) para aplicar la teoría de deformación discutida anteriormente.

La línea de mundo para BTZ es la siguiente

$$
d s^{2}=-n(r)^{2} d t^{2}+\frac{1}{n(r)^{2}} d r^{2}+r^{2}\left(n_{\phi}(r) d t+d \phi\right)^{2},
$$

donde $n(r)$ y $n_{\phi}(r)$ son funciones por determinar. 
Derivado de la métrica es fácil observar que relación guarda ésta con los elementos de la tétrada ${ }^{1}$ de modo que se cumpla

$$
d s^{2}=-\left(e_{\mu}^{0} d x^{\mu}\right)^{2}+\left(e_{\mu}^{1} d x^{\mu}\right)^{2}+\left(e_{\mu}^{2} d x^{\mu}\right)^{2}
$$

Para representar la línea de mundo en esta nueva base dada por la tétrada tenemos que considerar la métrica plana $\eta_{a b}$ de manera conveniente con la siguiente signatura $(-,+,+)$. Por lo tanto los elementos de la tétrada son

$$
\begin{aligned}
& e_{\mu}^{0}=(n(r), 0,0) \text {, } \\
& e_{\mu}^{1}=\left(\begin{array}{ll}
0, & \frac{1}{n(r)}, 0
\end{array}\right) \text {, } \\
& e_{\mu}^{2}=\left(r n_{\phi}(r), 0, \quad r\right) \text {. }
\end{aligned}
$$

Haciendo uso del programa realizado en Mathematica (btztng.nb), es posible obtener las ecuaciones de campo que determinarán la forma de las funciones $n(r)$ y $n_{\phi}(r)$ con el tensor de energía momento igual a cero, y considerando una teoría gravitacional con constante cosmológica. Esto nos conduce a las expresiones

$$
\begin{aligned}
& n(r)^{2}=\frac{J^{2}}{4 r^{2}}-4 \lambda^{2} r^{2}-M, \\
& n_{\phi}(r)=-\frac{J}{2 r^{2}},
\end{aligned}
$$

donde las constantes $M$ y $J$ están asociadas a la masa y momento angular del agujero negro respectivamente mientras que $\lambda$ está relacionado con la constante cosmológica.

Sustituyendo las funciones $n(r)$ y $n_{\phi}(r)$ de la ecuación (4.3) en (2.86), determinamos los campos de norma $\omega_{\mu}^{a b}$ diferentes de cero

$$
\begin{aligned}
& \omega_{\mu}^{01}=\left(\begin{array}{lll}
-4 \lambda^{2} r, \quad 0,-\frac{J}{2 r}
\end{array}\right), \\
& \omega_{\mu}^{02}=\left(\begin{array}{lll}
0, & -\frac{J}{r^{2} \sqrt{\frac{J^{2}}{r^{2}}-16 \lambda^{2} r^{2}-4 M}}, 0
\end{array}\right), \\
& \omega_{\mu}^{12}=\left(\begin{array}{lll}
0, & 0, & -\sqrt{\frac{J^{2}}{4 r^{2}}-4 \lambda^{2} r^{2}-M}
\end{array}\right) .
\end{aligned}
$$

Para la conformación final de los elementos de la tétrada deformada nos auxiliamos del programa realizado en Mathematica (btz_tng_def.nb), el cual nos permitió obtener la tétrada deformada, que a continuación presentamos:

${ }^{1}$ Observando la relación (2.73) es fácil determinar la tétrada correspondiente a una métrica dada 


$$
\begin{aligned}
& \hat{e}_{0}^{0}=\sqrt{\frac{J^{2}}{4 r^{2}}-4 \lambda^{2} r^{2}-M} \\
& +\theta^{2} \frac{3 J^{4} \lambda^{2}+J^{2}\left(32 \lambda^{4} r^{4}+8 \lambda^{2} M r^{2}+M^{2}\right)-16 \lambda^{2} r^{4}\left(4 \lambda^{2} r^{2}+M\right)^{2}}{32 r^{2} \sqrt{\frac{J^{2}}{4 r^{2}}-4 \lambda^{2} r^{2}-M}\left(4 r^{2}\left(4 \lambda^{2} r^{2}+M\right)-J^{2}\right)}, \\
& \hat{e}_{1}^{0}=\theta \frac{i J\left(8 \lambda^{2} r^{2}+M\right)}{\sqrt{\frac{J^{2}}{4 r^{2}}-4 \lambda^{2} r^{2}-M}\left(4 r^{2}\left(4 \lambda^{2} r^{2}+M\right)-J^{2}\right)}, \\
& \hat{e}_{2}^{0}=-\theta^{2} \frac{J^{3}-2 J M r^{2}}{64 r^{4} \sqrt{\frac{J^{2}}{4 r^{2}}-4 \lambda^{2} r^{2}-M}}, \\
& \hat{e}_{0}^{1}=\theta \frac{i J\left(8 \lambda^{2} r^{2}+M\right)}{8 r^{2} \sqrt{\frac{J^{2}}{4 r^{2}}-4 \lambda^{2} r^{2}-M}}, \\
& \hat{e}_{1}^{1}=\frac{1}{\sqrt{\frac{J^{2}}{4 r^{2}}-4 \lambda^{2} r^{2}-M}} \\
& -\theta^{2} \frac{1}{64 r^{4} \sqrt{\frac{J^{2}}{4 r^{2}}-M-4 \lambda^{2} r^{2}}\left(J^{2}-4 r^{2}\left(M+4 \lambda^{2} r^{2}\right)\right)^{2}}\left(-J^{6}\right. \\
& +4 J^{4}\left(3 M r^{2}+14 \lambda^{2} r^{4}\right)+8 J^{2} r^{4}\left(-M^{2}+16 \lambda^{2} M r^{2}+64 \lambda^{4} r^{4}\right) \\
& \left.+128 \lambda^{2} r^{8}\left(-M^{2}+8 \lambda^{2} M r^{2}+48 \lambda^{4} r^{4}\right)\right), \\
& \hat{e}_{2}^{1}=\theta \frac{i\left(8 \lambda^{2} r^{2}+M\right)}{4 \sqrt{\frac{J^{2}}{4 r^{2}}-4 \lambda^{2} r^{2}-M}}, \\
& \hat{e}_{0}^{2}=-\frac{J}{2 r}-\theta^{2} \frac{J\left(2 J^{2} \lambda^{2}+32 \lambda^{4} r^{4}+8 \lambda^{2} M r^{2}+M^{2}\right)}{32 r^{3}\left(4 \lambda^{2} r^{2}+M\right)-8 J^{2} r}, \\
& \hat{e}_{1}^{2}=-\theta \frac{i\left(J^{2}+16 \lambda^{2} r^{4}\right)}{4 J^{2} r-64 \lambda^{2} r^{5}-16 M r^{3}}, \\
& \hat{e}_{2}^{2}=r+\theta^{2} \frac{J^{2}}{32 r^{3}} \text {. }
\end{aligned}
$$

\subsection{Kerr-Newman No-Conmutativo}

Vamos a fijar ahora nuestra atención en aplicar la teoría de deformación para un hoyo negro con rotación lenta, en otras palabras tomaremos solo términos lineales del parámetro de rotación $a$.

Para ello utilizamos la métrica de Kerr-Newman [5] con rotación lenta que tiene la siguiente forma 


$$
\begin{aligned}
d s^{2}= & -G(r)\left(d t-\frac{a P(r) Q(\vartheta)}{2 G(r)} d \phi\right)^{2}+\frac{1}{G(r)} d r^{2}+r^{2} d \vartheta^{2} \\
& +r^{2} \sin ^{2}(\vartheta)\left(d \phi+\frac{a P(r) Q(\vartheta)}{2 r^{2} \sin ^{2}(\vartheta)} d t\right)^{2},
\end{aligned}
$$

donde $P(r), G(r)$ y $Q(\vartheta)$ son funciones conocidas de sus argumentos

De manera muy similar al caso de BTZ, identificamos los términos de la tétrada que corresponde a Kerr-Newman. Tenmos así

$$
\begin{aligned}
& e_{\mu}^{0}=\left(\sqrt{G(r)}, \quad 0, \quad 0,-\frac{a P(r) Q(\vartheta)}{2 \sqrt{G(r)}}\right), \\
& e_{\mu}^{1}=\left(\begin{array}{lll}
0, & \frac{1}{\sqrt{G(r)}}, 0, & 0
\end{array}\right) \text {, } \\
& e_{\mu}^{2}=(0,0, r, 0) \text {, } \\
& e_{\mu}^{3}=\left(\frac{a P(r) Q(\vartheta)}{2 r \sin (\vartheta)}, 0, \quad 0, \quad r \sin (\vartheta)\right) \text {. }
\end{aligned}
$$

Con la ayuda del programa realizado en Mathematica (kerr_newman_tng.nb) se resolvieron las ecuaciones de campo gravitacional con constante cosmológica en el vacío, por lo que las funciones que constituyen a la métrica tienen la forma siguiente despreciando términos de orden cuadrático en el parámetro de rotación a

$$
\begin{aligned}
G(r) & =1-\frac{2 G M}{r}-4 \lambda^{2} r^{2}, \\
P(r) & =-\frac{2 G M}{r}, \\
Q(\vartheta) & =\sin ^{2}(\vartheta),
\end{aligned}
$$

con $G$ como la constante de gravitación universal, $M$ la masa, $\lambda$ como la constante cosmológica.

Tomando en cuenta la forma de estas funciones, podemos determinar los términos diferentes de cero del campo de norma $\omega_{\mu}^{a b}$ 


$$
\begin{aligned}
& \omega_{\mu}^{01}=\left(\frac{G M}{r^{2}}-4 \lambda^{2} r, \quad 0, \quad 0,-\frac{2 G M a \sin ^{2}(\vartheta)}{r^{2}}\right), \\
& \omega_{\mu}^{02}=\left(\begin{array}{llll}
0, & 0, & 0, \frac{a G M \sin (\vartheta) \cos (\vartheta)}{r^{2} \sqrt{-\frac{2 G M}{r}-4 \lambda^{2} r^{2}+1}}
\end{array}\right), \\
& \omega_{\mu}^{03}=\left(0, \frac{a G M \sin (\vartheta)(r-3 G M)}{r^{3} \sqrt{-\frac{2 G M}{r}-4 \lambda^{2} r^{2}+1}\left(2 G M+4 \lambda^{2} r^{3}-r\right)},-\frac{a G M \cos (\vartheta)}{r^{2} \sqrt{-\frac{2 G M}{r}-4 \lambda^{2} r^{2}+1}}, \quad 0\right),
\end{aligned}
$$

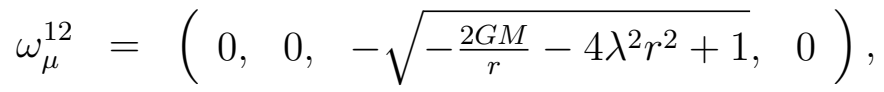

$$
\begin{aligned}
& \omega_{\mu}^{13}=\left(\frac{a G M \sin (\vartheta)\left(G M+8 \lambda^{2} r^{3}-r\right)}{r^{4} \sqrt{-\frac{2 G M}{r}-4 \lambda^{2} r^{2}+1}}, \quad 0, \quad 0,-\sin (\vartheta) \sqrt{-\frac{2 G M}{r}-4 \lambda^{2} r^{2}+1}\right), \\
& \omega_{\mu}^{23}=\left(\frac{2 a G M \cos (\vartheta)}{r^{3}}, 0, \quad 0,-\cos (\vartheta)\right) .
\end{aligned}
$$

Como se menciono casi al principio del capítulo, para Kerr-Newman se considera una matríz de deformación muy similar a la de BTZ (4.1) la cual es

$$
\theta^{\mu \nu}=\left[\begin{array}{cccc}
0 & 0 & 0 & 0 \\
0 & 0 & \theta & 0 \\
0 & -\theta & 0 & 0 \\
0 & 0 & 0 & 0
\end{array}\right]
$$

Esta elección es útil para simplificar los cálculos siguientes al mismo tiempo que captura las caraterísticas principales de la deformación.

Para la determinación de los elementos deformados nos apoyamos de un programa desarrollado en Mathematica (kerr_newman_tng_def.nb), que nos da la oportunidad de obtener los resultados siguientes de los elementos de la tétrada deformada 


$$
\begin{aligned}
& \hat{e}_{0}^{0}=\sqrt{-\frac{2 G M}{r}-4 \lambda^{2} r^{2}+1} \\
& +\frac{\theta^{2}\left(-11 G^{2} M^{2}+G M\left(6 r-28 \lambda^{2} r^{3}\right)-4 \lambda^{4} r^{6}+\lambda^{2} r^{4}\right)}{8 r^{4} \sqrt{-\frac{2 G M}{r}-4 \lambda^{2} r^{2}+1}}, \\
& \hat{e}_{3}^{0}=\frac{a G M \sin ^{2}(\vartheta)}{r \sqrt{-\frac{2 G M}{r}-4 r^{2} \lambda^{2}+1}}+\theta \frac{i a G M \sin (2 \vartheta)\left(12 G M+12 \lambda^{2} r^{3}-5 r\right)}{8 r^{2} \sqrt{-\frac{2 G M}{r}-4 \lambda^{2} r^{2}+1}\left(2 G M+4 \lambda^{2} r^{3}-r\right)} \\
& +\theta^{2} \frac{a G M}{32 r^{4} \sqrt{-\frac{2 G M}{r}-4 \lambda^{2} r^{2}+1}\left(-2 G M-4 \lambda^{2} r^{3}+r\right)^{2}}\left[(92-6 i) G^{3} M^{3}\right. \\
& +G^{2} M^{2} r\left((674-12 i) \lambda^{2} r^{2}-(163+10 i)\right)+\cos (2 \theta)\left((-92+6 i) G^{3} M^{3}\right. \\
& +G^{2} M^{2} r\left((155+14 i)-(674-12 i) \lambda^{2} r^{2}\right) \\
& -6 G M r^{2}\left(296 \lambda^{4} r^{4}-(128-32 i) \lambda^{2} r^{2}+(14-i)\right) \\
& \left.-2 r^{3}\left(688 \lambda^{6} r^{6}-(432-280 i) \lambda^{4} r^{4}+(96-80 i) \lambda^{2} r^{2}-(7-4 i)\right)\right) \\
& +8 G M r^{2}\left(222 \lambda^{4} r^{4}-(104+13 i) \lambda^{2} r^{2}+(12+2 i)\right) \\
& \left.+2 r^{3}\left(688 \lambda^{6} r^{6}-(480+24 i) \lambda^{4} r^{4}+(116+20 i) \lambda^{2} r^{2}-(9+2 i)\right)\right] \\
& \hat{e}_{1}^{1}=\frac{1}{\sqrt{-\frac{2 G M}{r}-4 \lambda^{2} r^{2}+1}}+\frac{v^{2}\left(-3 G^{2} M^{2}+G M r\left(2-21 \lambda^{2} r^{2}\right)+12 \lambda^{4} r^{6}+\lambda^{2} r^{4}\right)}{8 r^{3} \sqrt{-\frac{2 G M}{r}-4 \lambda^{2} r^{2}+1}\left(2 G M+4 \lambda^{2} r^{3}-r\right)}, \\
& \hat{e}_{2}^{1}=-\theta \frac{i\left(1-8 \lambda^{2} r^{2}\right)}{4 \sqrt{-\frac{2 G M}{r}-4 \lambda^{2} r^{2}+1}}, \\
& \hat{e}_{1}^{2}=-\theta \frac{i \lambda^{2} r^{2}}{2 G M+4 \lambda^{2} r^{3}-r}, \\
& \hat{e}_{2}^{2}=r-\theta^{2} \frac{G M\left(24 G M+72 \lambda^{2} r^{3}-13 r\right)}{32 r^{2}\left(2 G M+4 \lambda^{2} r^{3}-r\right)}, \\
& \hat{e}_{0}^{3}=-\frac{a G M \sin (\theta)}{r^{2}}+\theta \frac{i a G M \cos (\vartheta)\left(9 G M+12 \lambda^{2} r^{3}-4 r\right)}{2 r^{3}\left(2 G M+4 \lambda^{2} r^{3}-r\right)} \\
& -\theta^{2} \frac{a G M \sin (\vartheta)}{32 r^{5}\left(-2 G M-4 \lambda^{2} r^{3}+r\right)^{2}}\left[(124+12 i) G^{3} M^{3}\right. \\
& +G^{2} M^{2} r\left((856+24 i) \lambda^{2} r^{2}-(205-66 i)\right) \\
& +G M r^{2}\left(2304 \lambda^{4} r^{4}-(1100-344 i) \lambda^{2} r^{2}+(119-82 i)\right) \\
& \left.+(8-8 i) r^{3}\left((172+172 i) \lambda^{6} r^{6}-(140+72 i) \lambda^{4} r^{4}+(36+9 i) \lambda^{2} r^{2}-3\right)\right] \text {, }
\end{aligned}
$$




$$
\begin{aligned}
\hat{e}_{3}^{3}= & r \sin (\vartheta)-\frac{1}{4} i \theta \cos (\vartheta)-\theta^{2} \frac{(1-i) \sin (\vartheta)}{16 r^{2}\left(2 G M+4 \lambda^{2} r^{3}-r\right)}\left[(1+i) G^{2} M^{2}\right. \\
& +G M r\left((14+14 i) \lambda^{2} r^{2}-(1+2 i)\right) \\
& \left.+(1+i) \lambda^{2} r^{4}\left(-4 \lambda^{2} r^{2}+(1+2 i)\right)\right] .
\end{aligned}
$$

Las expresiones anteriores son cuadráticas en el parámetro de deformación $\theta$. Por medio de ellas es posible determinar las componentes de la métrica deformada por medio de la relación $(3.24)$. 



\section{Capítulo 5}

\section{Potencial Efectivo No-Conmutativo en BTZ}

En este capítulo vamos a utilizar la métrica deformada BTZ para determinar la forma del potencial efectivo deformado asociado al movimiento orbital de partículas prueba. Decidimos trabajar con esta métrica ya que los cálculos pueden ser realizados de manera exacta en el parámetro de rotación, algo que no sucede con la métrica de Kerr-Newman con rotación lenta. Para esto vamos a utilizar la relación (3.20) que define la forma de la métrica deformada; al existir un producto estrella se tiene que realizar dicho producto dada la relación (3.2)

$$
\hat{e}_{\mu}^{a}(r) \star \hat{e}_{\nu}^{b+}(r)=\hat{e}_{\mu}^{a}(r) \hat{e}_{\nu}^{b+}(r)+\left.\frac{i}{2} \theta^{\rho \tau} \frac{\partial}{\partial x^{\rho}} \frac{\partial}{\partial y^{\tau}} \hat{e}_{\mu}^{a}(r) \hat{e}_{\nu}^{b+}\left(r^{\prime}\right)\right|_{y=x}+\ldots(\mathcal{O})^{n}
$$

Derivado del cálculo de la tétrada para BTZ, es posible observar que solo se tiene dependencia en la coordenada $r$, por lo que en el cálculo del producto estrella entre tétradas solo contribuyen los términos a orden cero lo que implica que el cálculo de la métrica es

$$
\hat{g}_{\mu \nu}=\frac{1}{2} \eta_{a b}\left(\hat{e}_{\mu}^{a} \hat{e}_{\nu}^{b \dagger}+\hat{e}_{\mu}^{a \dagger} \hat{e}_{\nu}^{b}\right)
$$

Se observa que al tener dependencia solo en la variable $r$, el producto estrella se transforma en el producto normal que conocemos, ya que las derivadas cruzadas al actuar sobre las tétradas son igual a cero, esto se debe a que $\theta^{r \phi} \neq 0$. Sin embargo este producto involucra cantidades no conmutativas, $\hat{e}_{\mu}^{a}$, por lo que la métrica $\hat{g}_{\mu \nu}$ tendrá términos proporcionales a $\theta$. Usando el programa realizado en Mathematica (btz_tng_def) obtenemos los elementos de $\hat{g}_{\mu \nu}$ diferentes de cero. 


$$
\begin{aligned}
\hat{g}_{00}= & 4 \lambda^{2} r^{2}+M-\theta^{2} \frac{\lambda^{2}\left(4 r^{2}\left(4 \lambda^{2} r^{2}+M\right)-J^{2}\right)}{16 r^{2}}, \\
\hat{g}_{02}= & -\frac{J}{2}+\theta^{2} \frac{\left(J^{3}\left(M-8 \lambda^{2} r^{2}\right)-4 J\left(3 M^{2} r^{2}+28 \lambda^{2} M r^{4}+96 \lambda^{4} r^{6}\right)\right)}{32 r^{2}\left(4 r^{2}\left(M+4 \lambda^{2} r^{2}\right)-J^{2}\right)}, \\
\hat{g}_{11}= & \frac{1}{\frac{J^{2}}{4 r^{2}}-4 \lambda^{2} r^{2}-M} \\
& +\theta^{2} \frac{1}{16 r^{2}\left(4 r^{2}\left(M+4 \lambda^{2} r^{2}\right)-J^{2}\right)^{3}}\left[J^{6}-4 J^{4}\left(5 M r^{2}+32 \lambda^{2} r^{4}\right)\right. \\
& \left.+16 J^{2}\left(5 M^{2} r^{4}+56 \lambda^{2} M r^{6}+208 \lambda^{4} r^{8}\right)+256 \lambda^{2} r^{8}\left(M^{2}-4 \lambda^{2} M r^{2}-32 \lambda^{4} r^{4}\right)\right], \\
\hat{g}_{20}= & -\frac{J}{2}+\theta^{2} \frac{\left(J^{3}\left(M-8 \lambda^{2} r^{2}\right)-4 J\left(3 M^{2} r^{2}+28 \lambda^{2} M r^{4}+96 \lambda^{4} r^{6}\right)\right)}{32 r^{2}\left(4 r^{2}\left(M+4 \lambda^{2} r^{2}\right)-J^{2}\right)} \\
\hat{g}_{22}= & r^{2}+\theta^{2} \frac{\left(J^{4}-4 J^{2} r^{2}\left(M+4 \lambda^{2} r^{2}\right)+4 r^{4}\left(M+8 \lambda^{2} r^{2}\right)^{2}\right)}{16 r^{2}\left(J^{2}-4 r^{2}\left(M+4 \lambda^{2} r^{2}\right)\right)} .
\end{aligned}
$$

A partir de la métrica deformada $\hat{g}_{\mu \nu}$ podemos determinar el potencial efectivo. Para el cálculo tenemos que partir del Lagrangiano siguiente

$$
\hat{\mathcal{L}}=\frac{1}{2} \hat{g}_{\mu \nu} \frac{d x^{\mu}}{d \tau} \frac{d x^{\nu}}{d \tau},
$$

y para nuestro caso, $\hat{\mathcal{L}}$ es el Lagrangiano deformado derivado de (5.1) relacionado con la métrica deformada. Aquí $\tau$ es un parámetro afín a lo largo de la línea de mundo de una partícula de prueba. Entonces el Lagrangiano para BTZ deformado queda de la siguiente forma

$$
\begin{aligned}
\hat{\mathcal{L}}= & \frac{1}{2}\left(\frac{4 r^{2}\left(r^{\prime}\right)^{2}}{J^{2}-4 r^{2}\left(M+4 \lambda^{2} r^{2}\right)}-J t^{\prime} \phi^{\prime}+\left(t^{\prime}\right)^{2}\left(M+4 \lambda^{2} r^{2}\right)+r^{2}\left(\phi^{\prime}\right)^{2}\right) \\
& +\theta^{2} \frac{1}{32 r^{2}\left(4 r^{2}\left(M+4 \lambda^{2} r^{2}\right)-J^{2}\right)^{3}}\left[( r ^ { \prime } ) ^ { 2 } \left(J^{6}-4 J^{4}\left(5 M r^{2}+32 \lambda^{2} r^{4}\right)\right.\right. \\
& \left.+16 J^{2}\left(5 M^{2} r^{4}+56 \lambda^{2} M r^{6}+208 \lambda^{4} r^{8}\right)+256 \lambda^{2} r^{8}\left(M^{2}-4 \lambda^{2} M r^{2}-32 \lambda^{4} r^{4}\right)\right) \\
& +\left(J^{2}-4 r^{2}\left(M+4 \lambda^{2} r^{2}\right)\right)^{2}\left(J t ^ { \prime } \phi ^ { \prime } \left(J^{2}\left(M-8 \lambda^{2} r^{2}\right)\right.\right. \\
& \left.-4\left(3 M^{2} r^{2}+28 \lambda^{2} M r^{4}+96 \lambda^{4} r^{6}\right)\right)+\lambda^{2}\left(t^{\prime}\right)^{2}\left(J^{2}-4 r^{2}\left(M+4 \lambda^{2} r^{2}\right)\right)^{2} \\
& \left.\left.-\left(\phi^{\prime}\right)^{2}\left(J^{4}-4 J^{2} r^{2}\left(M+4 \lambda^{2} r^{2}\right)+4 r^{4}\left(M+8 \lambda^{2} r^{2}\right)^{2}\right)\right)\right],
\end{aligned}
$$

donde ' denota diferenciación con respecto a $\tau$.

$\mathrm{Al}$ solo tener coeficientes métricos dependientes de $r$ y $\theta$ en la métrica deformada es evidente que existan dos cantidades conservadas relacionadas con la energía y el momento 
angular. Estas cantidades pueden ser calculadas por medio de las relaciones siguientes

$$
\begin{aligned}
\hat{P}_{t}= & \frac{\partial \hat{\mathcal{L}}}{\partial \dot{t}}=-E \\
= & t^{\prime}\left(4 \lambda^{2} r^{2}+M\right)-\frac{J \phi^{\prime}}{2} \\
& +\frac{\theta^{2}}{32 r^{2}\left(4 r^{2}\left(4 \lambda^{2} r^{2}+M\right)-J^{2}\right)}\left[2 \lambda^{2} t^{\prime}\left(J^{2}-4 r^{2}\left(4 \lambda^{2} r^{2}+M\right)\right)^{2}\right. \\
& \left.+J \phi^{\prime}\left(J^{2}\left(M-8 \lambda^{2} r^{2}\right)-4\left(96 \lambda^{4} r^{6}+28 \lambda^{2} M r^{4}+3 M^{2} r^{2}\right)\right)\right],
\end{aligned}
$$

y

$$
\begin{aligned}
\hat{P}_{\phi}= & \frac{\partial \hat{\mathcal{L}}}{\partial \dot{\phi}}=L \\
= & r^{2} \phi^{\prime}-\frac{J t^{\prime}}{2}-\frac{\theta^{2}}{32 r^{2}\left(4 r^{2}\left(4 \lambda^{2} r^{2}+M\right)-J^{2}\right)}\left[t ^ { \prime } \left(4 J \left(96 \lambda^{4} r^{6}+28 \lambda^{2} M r^{4}\right.\right.\right. \\
& \left.\left.+3 M^{2} r^{2}\right)-J^{3}\left(M-8 \lambda^{2} r^{2}\right)\right) \\
& \left.+2 \phi^{\prime}\left(J^{4}-4 J^{2} r^{2}\left(4 \lambda^{2} r^{2}+M\right)+4 r^{4}\left(8 \lambda^{2} r^{2}+M\right)^{2}\right)\right] .
\end{aligned}
$$

La forma del Hamiltoniano se deriva de la teoría Lagrangiana bajo la relación $\hat{\mathcal{H}}=$ $\hat{P}_{\mu} x^{\prime \mu}-\hat{\mathcal{L}}$. Al cálcular el Hamiltoniano para BTZ nos damos cuenta que $\hat{\mathcal{L}}=\hat{\mathcal{H}}$, por lo que el Hamiltoniano es una constante de movimiento que relacionaremos con geodésicas tipo tiempo, tipo espacio o nulas

$$
\hat{\mathcal{H}}=\frac{1}{2} \hat{g}_{\mu \nu} \frac{d x^{\mu}}{d \tau} \frac{d x^{\nu}}{d \tau}=-\epsilon,
$$

dependiendo de si $\epsilon=+1,-1$ ó 0 respectivamente.

Para la determinación del potencial efectivo requerimos de utilizar las relaciones (5.4) y (5.5) en el lagrangiano (5.3) de tal modo que podamos escribir la ecuación geodésica para la coordenada $r$ en la forma siguiente [23]

$$
\left(\frac{\partial r}{\partial \tau}\right)^{2}=\left(E-\hat{\mathcal{V}}^{+}(r)\right)\left(E-\hat{\mathcal{V}}^{-}(r)\right) .
$$

Los potenciales $\hat{\mathcal{V}}^{ \pm}(r)$ son los potenciales efectivos que rigen el movimiento orbital de partículas prueba.

Para la presentación del potencial solo vamos a tomar en consideración términos lineales y cuadráticos en $\theta$. Como la expresión es extensa, la reescribimos como el cociente de dos funciones $g^{ \pm}(r)$ y $h(r)$ tales que

$$
\hat{\mathcal{V}}^{ \pm}=\frac{g^{ \pm}(r)}{h(r)} .
$$


Tenemos así que

$$
\begin{aligned}
g^{ \pm}(r)= & 64 J L r^{4}\left(J^{2}-4 r^{2}\left(M+4 \lambda^{2} r^{2}\right)\right)^{2} \\
& +4 \theta^{2} J L r^{2}\left[J^{4}\left(M-8 \lambda^{2} r^{2}\right)-16 J^{2} r^{2}\left(M^{2}+6 \lambda^{2} M r^{2}+16 \lambda^{4} r^{4}\right)\right. \\
& \left.+16 r^{4}\left(3 M^{3}+40 \lambda^{2} M^{2} r^{2}+208 \lambda^{4} M r^{4}+384 \lambda^{6} r^{6}\right)\right] \\
& \pm \sqrt{2}\left[-2048 r^{8}\left(L^{2}+2 r^{2} \epsilon\right)\left(4 r^{2}\left(M+4 \lambda^{2} r^{2}\right)-J^{2}\right)^{5}\right. \\
& +256 \theta^{2} r^{6}\left(J^{2}-4 r^{2}\left(M+4 \lambda^{2} r^{2}\right)\right)^{3}\left(J^{6} \epsilon-J^{4}\left(L^{2}\left(M+14 \lambda^{2} r^{2}\right)\right.\right. \\
& \left.+10 r^{2} \epsilon\left(M+6 \lambda^{2} r^{2}\right)\right)-4 J^{2}\left(L^{2} r^{2}\left(M^{2}+16 \lambda^{2} M r^{2}+80 \lambda^{4} r^{4}\right)\right. \\
& \left.+r^{4} \epsilon\left(-3 M^{2}-16 \lambda^{2} M r^{2}+32 \lambda^{4} r^{4}\right)\right) \\
& -8 r^{4}\left(M+4 \lambda^{2} r^{2}\right)\left(L^{2}\left(M^{2}+12 \lambda^{2} M r^{2}+48 \lambda^{4} r^{4}\right)\right. \\
& \left.\left.\left.+4 r^{2} \epsilon\left(M^{2}+14 \lambda^{2} M r^{2}+56 \lambda^{4} r^{4}\right)\right)\right)\right]^{1 / 2},
\end{aligned}
$$

y además

$$
\begin{aligned}
h(r)= & 128 r^{6}\left(J^{2}-4 r^{2}\left(4 \lambda^{2} r^{2}+M\right)\right)^{2}+8 r^{2} \theta^{2}\left[J^{6}-8 J^{4} r^{2}\left(4 \lambda^{2} r^{2}+M\right)\right. \\
& \left.+4 J^{2}\left(128 \lambda^{4} r^{8}+48 \lambda^{2} M r^{6}+5 M^{2} r^{4}\right)-16 r^{6}\left(4 \lambda^{2} r^{2}+M\right)\left(8 \lambda^{2} r^{2}+M\right)^{2}\right] .
\end{aligned}
$$

Al tener la forma final del potencial deformado podemos realizar una comparación gráfica del potencial deformado y el potencial sin deformar. Como una acotación importante, para el análisis de las gráficas del potencial efectivo, vamos a considerar un espacio adS, por lo tanto tenemos que $-\Lambda=4 \lambda^{2}$.

De forma general se van a presentar las gráficas del potencial efectivo con parámetro de rotación $J=0$, tanto para una partícula masiva como para una partícula nula, con diferentes valores para la masa del hoyo negro BTZ. 


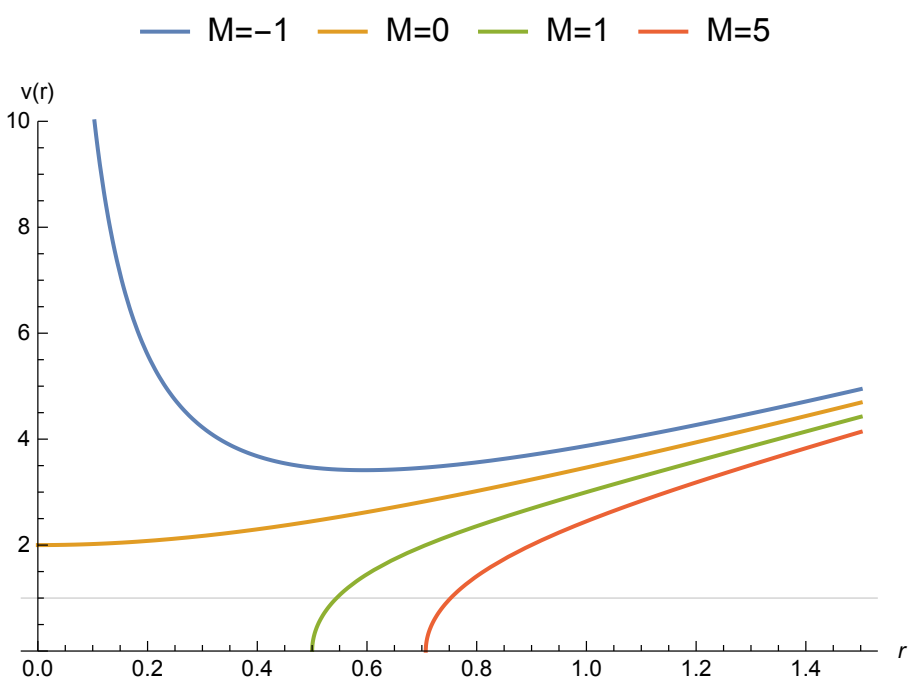

Figura 5.1: Comparación del potencial efectivo $\hat{\mathcal{V}}^{ \pm}$sin rotación y sin deformación para una partícula masiva, a diferentes valores de la masa del hoyo negro BTZ, con los siguientes valores: $J=0, L=1, \epsilon=1, \theta=0$

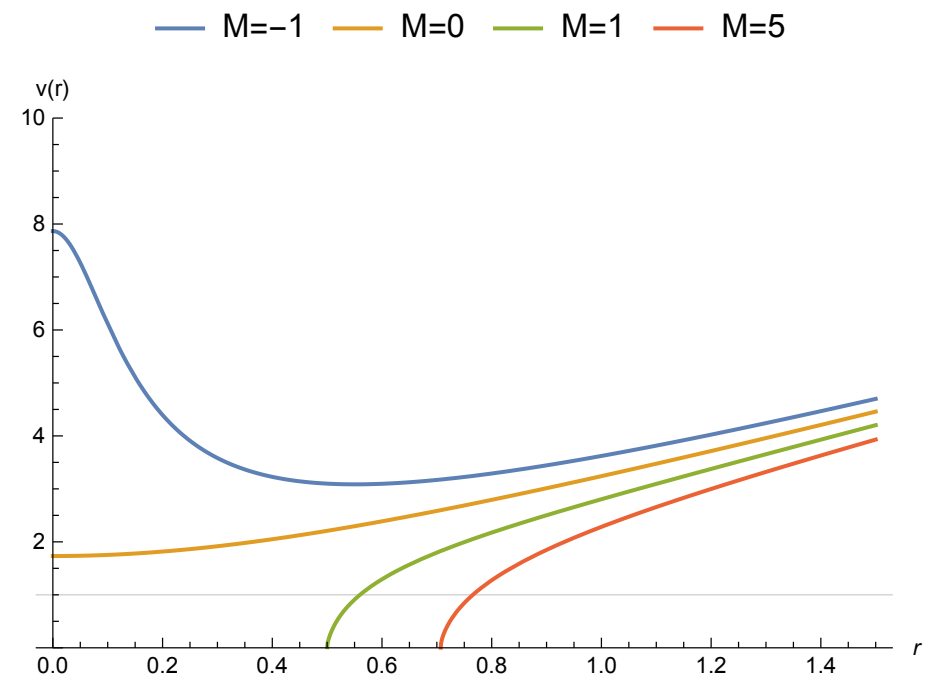

Figura 5.2: Comparación del potencial efectivo $\hat{\mathcal{V}}^{ \pm}$sin rotación y con deformación para una partícula masiva, a diferentes valores de la masa del hoyo negro BTZ, con los siguientes valores: $J=0, L=1, \epsilon=1, \theta=0,5$

De las figuras 5.1 y 5.2 que representan un hoyo negro para un partícula masiva, es evidente que para ambos casos deformado y sin deformar tenemos que la partícula queda confinada a una órbita periódica finita $[24,25,26]$. Al no encontrar ninguna diferencia significativa para valores de la masa del hoyo negro BTZ mayores de cero, se hace uso de un valor de la masa $M=-1$, solo con el fin de observar para que valores tenemos una diferencia entre la teoría deformada y sin deformar del hoyo negro BTZ, para el cual la partícula masiva puede acercarse al centro siempre y cuando tenga una energía $E$ mayor a una energía $E c$ crítica: 
es necesario mencionar que una masa negativa para BTZ esta relacionada con transiciones de fase [27] tema que no será tratado en este trabajo, sin embargo para el hoyo negro sin deformar no es posible que la partícula caiga al centro del agujero negro.

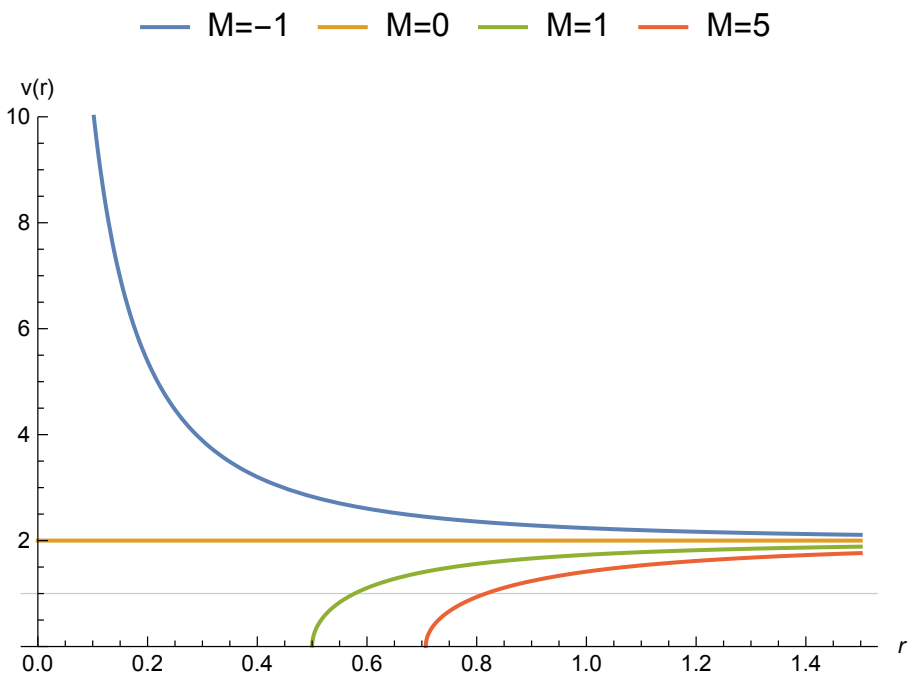

Figura 5.3: Comparación del potencial efectivo $\hat{\mathcal{V}}^{ \pm}$sin rotación y sin deformación para una partícula nula, a diferentes valores de la masa del hoyo negro BTZ, con los siguientes valores; $J=0, L=1, \epsilon=0, \theta=0$.

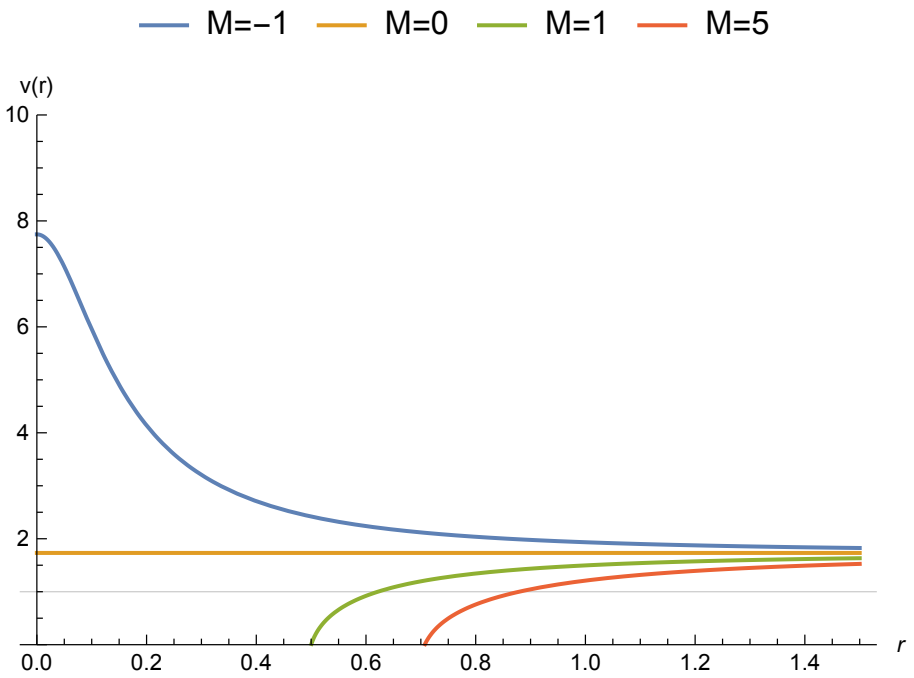

Figura 5.4: Comparación del potencial efectivo $\hat{\mathcal{V}}^{ \pm}$sin rotación y con deformación para una partícula nula, a diferentes valores de la masa del hoyo negro BTZ, con los siguientes valores; $J=0, L=1, \epsilon=0, \theta=0,5$.

En las figuras 5.3 y 5.4 observamos que tanto para un hoyo negro deformado y sin deformar la partícula nula presenta una órbita de escape, por lo cual vamos a detallar algunas diferencias. Para una masa $M=-1$ del hoyo negro BTZ observamos que para el hoyo negro 
sin deformar la partícula nula no puede aproximarse al centro del agujero negro, caso contrario cuando se tiene un hoyo negro deformado, para este caso se tiene la posibilidad de que la partícula pueda caer en el centro del hoyo negro para un valor de la energía $E$ mayor a una energía Ec crítica. Para los valores de la masa del hoyo negro BTZ $M \geq 0$ en ambas teorías tenemos prácticamente la misma forma del potencial.

A continuación se presenta el potencial efectivo para algunos valores en específico de los parámetros del hoyo negro BTZ. De las siguientes gráficas es posible notar que el potencial efectivo tiene diferentes comportamientos, ya sea para una partícula masiva o nula para un hoyo negro con rotación. Para realizar las graficas es necesario escoger un valor adecuado para $J$, por lo que vamos a considerar los valores para el horizonte del hoyo negro sin deformar ${ }^{1}$, que se define de la siguiente manera

$$
r^{ \pm}=\sqrt{-\frac{M}{8 \lambda^{2}}\left(1 \pm \sqrt{1+\frac{4 J^{2} \lambda^{2}}{M^{2}}}\right)} .
$$

De la relación anterior observamos que $M>0 \mathrm{y}|J| \leq M \sqrt{-\frac{1}{4 \lambda^{2}}}$, de este modo tenemos que el valor para $J$ queda restringido por los valores de la masa $M$ y la constante cosmológica $\lambda$.

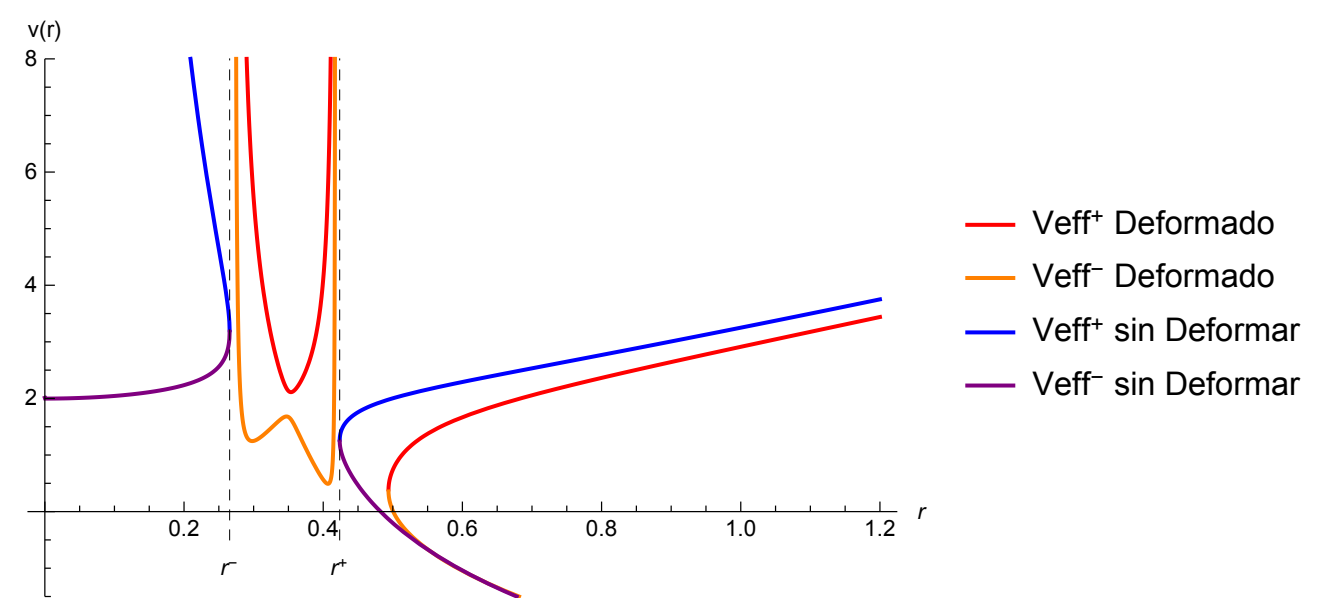

Figura 5.5: Comparación del potencial deformado contra el potencial sin deformar, con los siguientes valores $M=1, J=0,45, L=1, \epsilon=1, \theta=0,5$. (para una partícula masiva)

En la figura 5.5 se observa la comparación del potencial efectivo sin deformar con el potencial efectivo deformado con rotación $J=0,45$ para una partícula masiva. Para ambos casos el hoyo negro mantiene a la partícula masiva atrapada, lo cual nos dice que no existen órbitas de escape. Para un hoyo negro sin deformación vemos que también se presentan órbitas periódicas de colapso, dando la posibilidad a la partícula de caer al centro del hoyo negro. Para el caso de un hoyo negro deformado vemos que existe la presencia de órbitas

\footnotetext{
${ }^{1} \mathrm{Al}$ observar el componente de la métrica $\hat{g}_{11}$, vemos que las singularidades corresponden a $r$ y a $n(r)$, por lo que el horizonte sin deformar, será el mismo para el horizonte deformado
} 
periódicas de colapso y órbitas periódicas, ya que tenemos parte del potencial efectivo entre los horizontes $r^{ \pm}$.

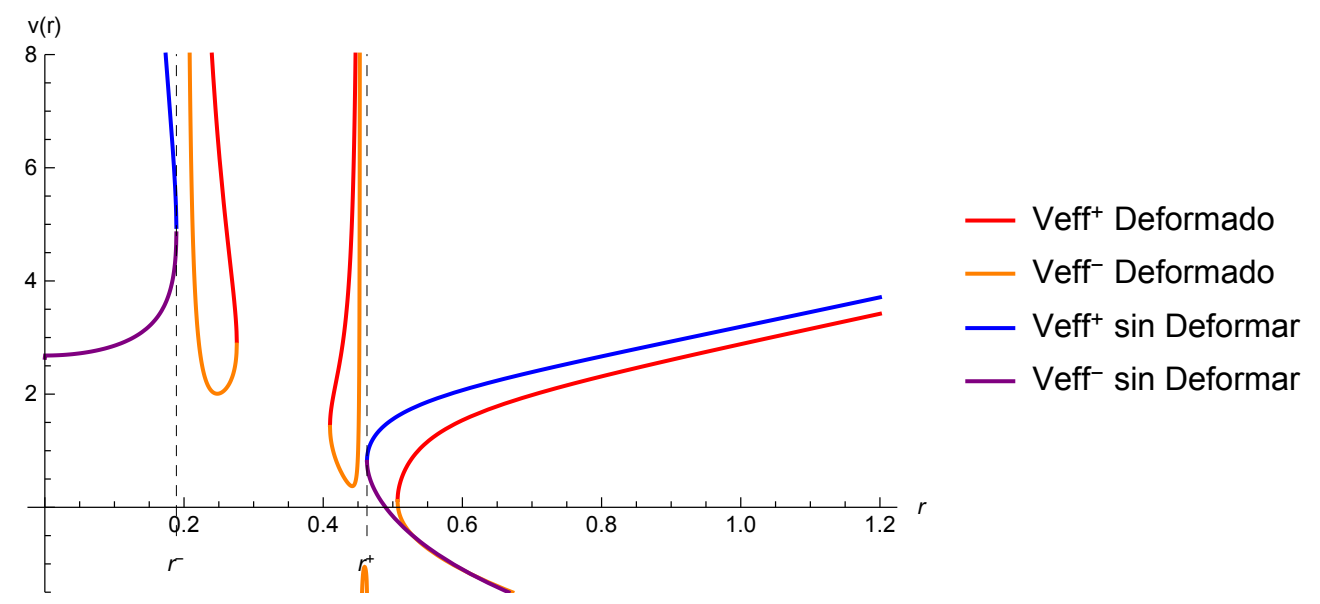

Figura 5.6: Comparación del potencial deformado contra el potencial sin deformar, con los siguientes valores $M=1, J=0,35, L=1, \epsilon=1, \theta=0,5$. (para una partícula masiva)

En la figura 5.6 se muestra una comparación del potencial efectivo deformado y sin deformar con rotación lenta $J=0,35$ para una partícula masiva. Vemos que el hoyo negro sin deformar tiene un comportamiento similar al de la figura 5.5. Para el hoyo negro deformado si tenemos algunas diferencias, las cuales radican en la forma del potencial que esta comprendido entre los horizontes $r^{ \pm}$, lo que permite considerar una órbita de extensón finita entre ambos horizontes, esto sucede como una consecuencia en la disminución de la rotación del hoyo negro.

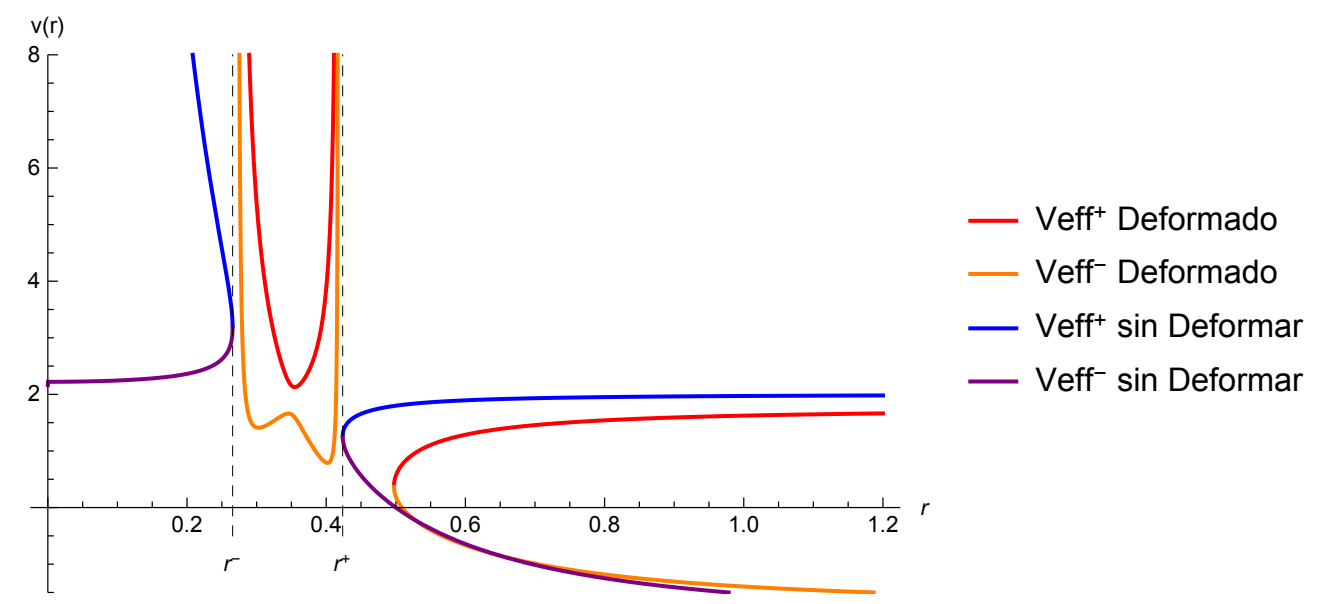

Figura 5.7: Comparación del potencial deformado contra el potencial sin deformar, con los siguientes valores $M=1, J=0,45, L=1, \epsilon=0, \theta=0,5$. (para una partícula nula) 


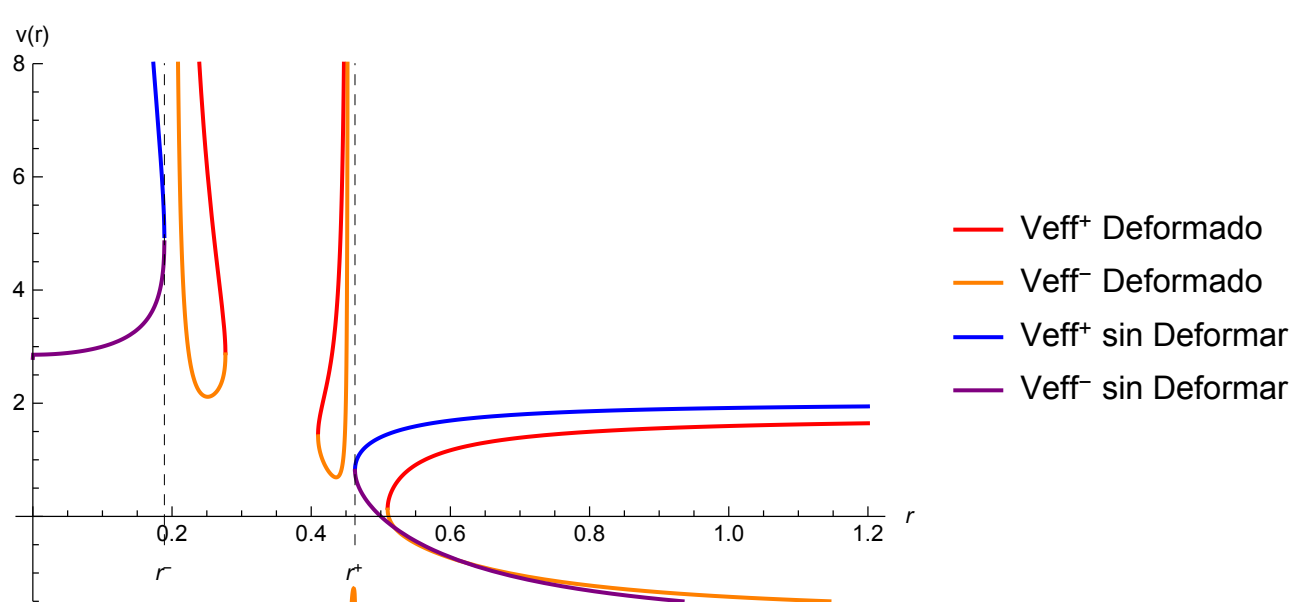

Figura 5.8: Comparación del potencial deformado contra el potencial sin deformar, con los siguientes valores $M=1, J=0,35, L=1, \epsilon=0, \theta=0,5$. (para una partícula nula)

En las figuras 5.7 y 5.8 tenemos la comparación del potencial efectivo y sin deformar para una partícula nula con valores para la rotación $J=0,45$ y $J=0,35$. Se observa que práticamente se tienen los mismos comportamientos que en las dos figuras anteriores $5.5 \mathrm{y}$ 5.6 con la diferencia que es posible tener órbitas de escape y de colapso. 



\section{Capítulo 6}

\section{Conclusiones y Perspectivas}

Como una aportación del presente trabajo se determinaron los campos de norma noconmutativos para la teoría gravitacional de la relatividad general en 4 y 3 dimensiones espacio-temporales, a partir de la ayuda del formalismo de Eli Cartán y el grupo de Poincaré como una simetría interna. Bajo este procedimiento fue posible obtener el campo de norma gravitacional $A_{\mu}=e_{\mu}^{a} P_{a}+\frac{1}{2} \omega_{\mu}^{a b} M_{a b}$, en el cual se utilizó la aplicación de Seiberg-Witten ampliamente usada en la construcción de teorías de norma no-conmutativas para la determinación de los campos de norma no-conmutativos asociados al vierbein o tétrada $\hat{e}_{\mu}^{a}$ y la conexión $\hat{\omega}_{\mu}^{a b}$. Con el desarrollo de esta teoría gravitacional no-conmutativa se obtuvo la expresión de la métrica para los agujeros negros BTZ [4], Kerr-Newman [5] en 3 y 4 dimensiones espacio-temporales respectivamente con relaciones no-conmutativas entre las coordenadas $r$, $\phi$ en BTZ y $r, \vartheta$ en Kerr-Newman a segundo orden en el parámetro de deformación $\theta$. Cabe mencionar que existen publicaciones previas que presentan hoyos negros deformados como lo son Schwarzschild [21], donde se obtiene la solución deformada sobre los campos de norma con un tensor de energía momento diferente de cero y BTZ [28, 29, 30] este último haciendo uso de la teoría de Chern-Simons [31] como una teoría de norma equivalente a la teoría gravitacional de Einstein 3-dimensional. Los anteriores hoyos negros no-conmutativos usan la aplicación de Seiberg-Witten para la obtención de la solución no-conmutativa.

Por la facilidad que presenta trabajar en dimensiones menores, se eligió la métrica BTZ no-conmutativa para determinar la forma del potencial efectivo deformado del movimiento orbital de partículas prueba. Para la métrica BTZ no-conmutativa se obtuvieron las cantidades conservadas de la energía $P_{t}$ y el momento angular $P_{\phi}$, el potencial efectivo del movimiento orbital de partículas prueba así como su representación gráfica que fueron comparadas con la teoría sin deformar, para distintos valores de la masa del hoyo negro y rotación. Los casos analizados fueron una partícula masiva y otra no masiva. En el caso de la partícula masiva observamos de las figuras 5.1 y 5.2 el potencial efectivo sin rotación la existencia de órbitas peródicas, con la diferencia que para el potencial deformado con una masa negativa del agujero negro, la partícula puede acercarse a su centro para un valor de la energía mayor a una energía crítica. Cuando se tiene una rotación diferente de cero para el mismo caso de la partícula masiva se observa de las figuras 5.5 y 5.6 que para un hoyo negro sin deformar existen órbitas periódicas de colapso permitiendo a la partícula caer al centro del agujero negro. Para el hoyo negro deformado se observa la existencia de órbitas periódicas de colapso pero a diferencia del caso conmutativo, entre los horizontes $r^{ \pm}$se presentan órbitas periódicas, que 
al disminuir la rotación del hoyo negro permiten la posibilidad de que las partículas caigan al centro del hoyo negro. Para el caso de la partícula no masiva tenemos un comportamiento similar al de la partícula masiva, con la diferencia de que existen órbitas de escape además de las de colapso.

En todo trabajo de tesis, siempre existirán preguntas o formulaciones que deriven ya sea en un nuevo trabajo de tesis, o bien un complemento al mismo. Por tal motivo se exponen las siguientes inquietudes:

1. ¿Qué dificultades se harían presentes para el cálculo de las trayectorias geodésicas BTZ y Kerr-Newman no-conmutativas?

2. ¿Qué características presentaría el potencial efectivo deformado de partículas prueba en la métrica de Kerr-Newman?

El interés en la formulación de las preguntas anteriores surge por la posibilidad de encontrar en las trayectorias geodésicas algo particular o característico al tener proximidad de una partícula masiva y no masiva al agujero negro que nos dé más información para una teoría gravitacional no-conmutativa y poder trascender el tratamiento perturbativo. El estudio de teorías no conmutativas es significativo para el desarrollo de una teoría de gravedad cuántica donde la deformación de la geometría del espacio-tiempo corresponda a su cuantización. Esta tarea es demasiado grande y ambiciosa, y nosotros solo hemos estudiado algunos posibles efectos en el caso particular de un modelo en 3 y 4 dimensiones espacio-temporales. 


\section{Bibliografía}

[1] K. Moriyasu, An Elementary Primer For Gauge Theory. Farrer Road, Singapore: World Scientific Publishing Co Pte Ltd., 1983, págs. 1-70.

[2] W. Heisenberg, "Uber den anschaulichen Inhalt der quantentheoretischen Kinematik und Mechanik", Z. Phys., vol. 43, págs. 172-198, 1927. DOI: 10.1007/BF01397280.

[3] H. S. Snyder, "Quantized Space-Time", Physical Review, vol. 71, n. ${ }^{\circ}$ 1, págs. 38-41, mayo de 1946.

[4] M. Bañados, C. Teitelboim y J. Zanelli, "Black Hole in Three-Dimensional Spacetime", Physical Review Letters, vol. 69, n. ${ }^{\circ}$ 13, págs. 1849-1851, sep. de 1992.

[5] S. Carroll, Space Time and Geometry: An introduction to general relativity. San Francisco: Addison Wesley, 2004, cap. More General Black Holes, págs. 261-265.

[6] K. Ulker y B. Yapiskan, "Seiberg-Witten maps to all orders", Phys. Rev., vol. D77, pág. 065 006, 2008. DOI: 10.1103/PhysRevD.77.065006. arXiv: 0712.0506 [hep-th].

[7] H. Weyl, "Eine neue Erweiterung der Relativitätstheorie", Annalen der Physik, vol. 364, n. ${ }^{\circ}$ 10, págs. 101-133, 1919.

[8] C. N. Yang y R. L. Mills, "Conservation of Isotopic Spin and Isotopic Gauge Invariance", Physical Review, vol. 96, n. ${ }^{\circ}$ 1, págs. 191-195, oct. de 1945.

[9] T. L. Curtright y C. K. Zachos, "Quantum Mechanics in Phase Space", Asia Pac. Phys. Newslett., vol. 1, págs. 37-46, 2012. DOI: 10.1142/S2251158X12000069. arXiv: 1104.5269 [physics.hist-ph].

[10] H. S. Snyder, "The Electromagnetic Field in Quatized Space-Time", Physical Review, vol. 72 , n. ${ }^{\circ}$ 1, págs. $68-71$, jul. de 1947.

[11] B. Jurčo, Noncommutative Gauge Theories via Seiberg-Witten Map. Berlin, Heidelberg: Springer Berlin Heidelberg, 2005, págs. 209-217.

[12] J. D. Jackson, Classical Electrodynamics. New York: John Wiley y Sons, Inc., 1962, cap. Time-Varying Fields, Maxwell's Equations, Conservation Laws, págs. 170-198, 641 págs.

[13] L. N. Chang, K. I. Macrae y F. Mansouri, "Geometrical approach to local gauge and supergauge invariance: Local gauge theories and supersymmetric strings*", Phys. Rev. D, vol. 13, n. ${ }^{\circ}$ 2, págs. 235-249, ene. de 1976.

[14] S. Chandrasekhar, The Mathematical Theory of Black Holes. New York: Clarendon Press, 1983, cap. Mathematical Preliminaries, págs. 3-40, 646 págs. 
[15] G. Zet, V. Manta, S. O. Iradinschi y B. Ciobanu, "A computer aided study of deSitter gauge theory of gravitation", Mathematical and Computer Modelling, vol. 43, págs. 458-465, 2006. DOI: https://doi.org/10.1016/j.mcm.2005.10.008.

[16] M. Blagojević, Gravitation and Gauge Symmetries. London: IOP Publishing Ltd, 2002, cap. Poincaré gauge theory, págs. 42-75, 522 págs.

[17] B. Jurco, S. Schraml, P. Schupp y J. Wess, "Enveloping algebra valued gauge transformations for nonAbelian gauge groups on noncommutative spaces", Eur. Phys. J., vol. C17, págs. 521-526, 2000. DOI: 10.1007/s100520000487. arXiv: hep-th/0006246 [hep-th].

[18] N. Seiberg y E. Witten, "String theory and noncommutative geometry", JHEP, vol. 09, pág. 032, 1999. DOI: 10 .1088/1126-6708/1999/09/032. arXiv: hep-th/9908142 [hep-th].

[19] L. Moller, "Second order of the expansions of action functionals of the noncommutative standard model", JHEP, vol. 10, pág. 063, 2004. DOI: 10.1088/1126-6708/2004/10/ 063. arXiv: hep-th/0409085 [hep-th].

[20] B. Jurco, L. Moller, S. Schraml, P. Schupp y J. Wess, "Construction of nonAbelian gauge theories on noncommutative spaces", Eur. Phys. J., vol. C21, págs. 383-388, 2001. DOI: 10.1007/s100520100731. arXiv: hep-th/0104153 [hep-th].

[21] M. Chaichian, A. Tureanu y G. Zet, "Corrections to Schwarzschild solution in noncommutative gauge theory of gravity", Phys. Lett., vol. B660, págs. 573-578, 2008. DOI: 10.1016/j.physletb.2008.01.029. arXiv: 0710.2075 [hep-th].

[22] A. H. Chamseddine, "Deforming Einstein's gravity", Phys. Lett., vol. B504, págs. 33-37, 2001. DOI: 10.1016/S0370-2693(01)00272-6. arXiv: hep-th/0009153 [hep-th].

[23] N. Cruz, C. Martnez y L. Pea, "Geodesic structure of the $(2+1)$-dimensional BTZ black hole", Classical and Quantum Gravity, vol. 11, n. ${ }^{\circ} 11$, pág. 2731, 1994. dirección: http://stacks.iop.org/0264-9381/11/i=11/a=014.

[24] E. Hackmann, C. Lammerzahl, V. Kagramanova y J. Kunz, "Analytical solution of the geodesic equation in Kerr-(anti) de Sitter space-times", Phys. Rev., vol. D81, pág. 044 020, 2010. DOI: 10.1103/PhysRevD.81.044020. arXiv: 1009.6117 [gr-qc].

[25] R. Linares, M. Maceda y D. Martnez-Carbajal, "Test Particle Motion in the Born-Infeld Black Hole", Phys. Rev., vol. D92, n. ${ }^{\circ}$ 2, pág. 024 052, 2015. DOI: 10.1103/PhysRevD. 92.024052. arXiv: 1412.3569 [gr-qc].

[26] D. M. Carbajal, "Geodésicas en Agujeros Negros de Einstein-Born-Infeld", M.Sc. thesis, Universidad Auntónoma Metropolitana Unidad Iztapalapa, jun. de 2013, cap. Geodésicas en agujeros negros de EBI, pág. 126.

[27] Y. S. Myung, "Phase transition between the BTZ black hole and AdS space", Phys. Lett., vol. B638, págs. 515-518, 2006. DOI: 10.1016/j .physletb. 2006.04.024. arXiv: gr-qc/0603051 [gr-qc]. 
[28] J. Sadeghi y V. R. Shajiee, "Effective Potential in Noncommutative BTZ Black Hole", International Journal of Theoretical Physics, vol. 55, n. ${ }^{\circ}$ 2, págs. 892-900, feb. de 2016, ISSN: 1572-9575. DOI: 10.1007/s10773-015-2732-x. dirección: https : //doi .org/ 10.1007/s10773-015-2732-x.

[29] E. Chang-Young, D. Lee e Y. Lee, "Noncommutative BTZ Black Hole in Polar Coordinates", Class. Quant. Grav., vol. 26, pág. 185 001, 2009. DOI: 10.1088/0264-9381/ 26/18/185001. arXiv: 0808.2330 [hep-th].

[30] S. Cacciatori, D. Klemm, L. Martucci y D. Zanon, "Noncommutative Einstein-AdS gravity in three-dimensions", Phys. Lett., vol. B536, págs. 101-106, 2002. DOI: 10 . 1016/S0370-2693(02)01823-3. arXiv: hep-th/0201103 [hep-th].

[31] S. Carlip, "Lectures on $(2+1)$ dimensional gravity", J. Korean Phys. Soc., vol. 28, S447-S467, 1995. arXiv: gr-qc/9503024 [gr-qc]. 


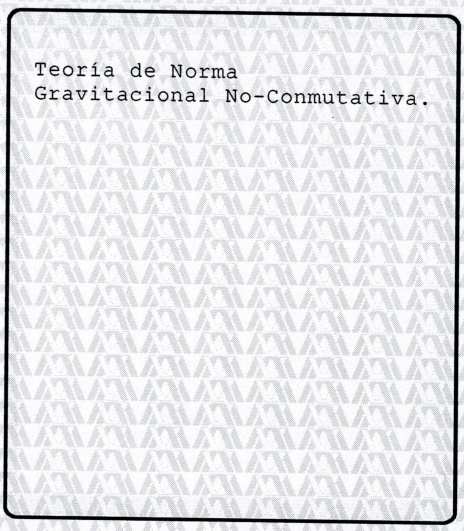

\begin{tabular}{|l}
\hline En la Ciudad de México, se presentaron a las 12:00 horas \\
del día del mes de diciembre del año 2018 en ia Unidad \\
Iztapalapa de la Universidad Autónoma Metropolitana, los \\
suscritos miembros del jurado: \\
DRA. NORA EVA BRETON BAEZ \\
DR. HUGO AURELIO MORAES TECOTL \\
DR. MARCO ANTONIO MACEDA SANTAMARIA
\end{tabular}

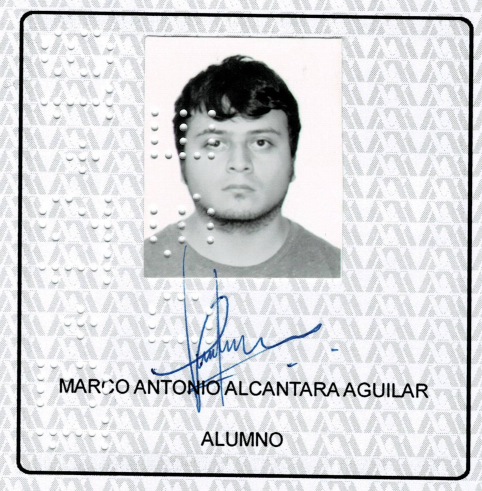

Bajo la Presidencia de la primera y con carácter de Secretario el último, se reunieron para proceder al Examen de Grado cuya denominación aparece al margen, para la

MAESTRO EN CIENCIAS (FISICA)

DE: MARCO ANTONIO ALCANTARA AGUILAR y de acuerdo con el artículo 78 fracción III del
Reglamento de Estudios Superiores de la Universidad
Autónoma Metropolitana, los miembros del jurado
resolvieron:

\section{Aprobar}

Acto continuo, la presidenta del jurado comunicó al interesado el resultado de la evaluación y, en caso DR. JOSE ANTONIODE YS REYES HEREDIA aprobatorio, le fue tomada la protesta. 